\title{
Estimation of climate change damage functions for 140 regions in the GTAP9 database
}

\author{
November 2015
}

\begin{abstract}
Climate change damage (or, more correctly, impact) functions relate variations in temperature (or other climate variables) to economic impacts in various dimensions, and are at the basis of quantitative modeling exercises for the assessment of climate change policies. This document provides a summary of results from a series of meta-analyses aimed at estimating parameters for six specific damage functions, referring to: sea level rise, agricultural productivity, heat effects on labor productivity, human health, tourism flows and households' energy demand. All parameters of the damage functions are estimated for each of the 140 countries and regions in the GTAP9 dataset.

To illustrate the salient characteristics of our estimates, we approximate the change in real GDP for the different effects, in all regions, corresponding to an increase in average temperature of $+3^{\circ} \mathrm{C}$. After considering the overall impact, we highlight which factor is the most significant one in each country, and we elaborate on the distributional consequences of climate change.
\end{abstract}

\section{Keywords:}

Climate change, integrated assessment, computable general equilibrium, damage function, climate impacts. 


\section{Introduction}

Understanding how the ongoing climate change could ultimately affect our society and the well-being of current and future generations requires an evaluation of the complex interplay between human and natural systems.

The human or anthropogenic influence on the earth climate is mainly associated with the emissions of greenhouse gases in the atmosphere, which is in turn related to the level of several economic activities. To forecast future climate, physical scientists need to know how many man-made GHG emissions should be expected, which depend on scenarios of economic growth as well as on the possible implementation of climate mitigation policies. On the other hand, economic growth itself may also be influenced by the climate change through its manifold impacts. As Tol (2015) puts it: "There are so many and so different effects: crops hit by worsening drought, crops growing faster because of carbon dioxide fertilization, heat stress increasing, cold stress decreasing, sea levels rising, cooling energy demand going up, heating energy demand going down, infectious disease spreading, and species going extinct. It is hard to make sense of this. Therefore, aggregate indicators are needed to assess whether climate change is, on balance, a good thing or a bad thing and whether the climate problem is small or large relative to the many other problems that we have.".

Damage functions have been introduced to this purpose, that is to "translate" physical impacts in terms of economic variables inside CGE, IAM and other numerical models. Therefore, damage functions are one ore more relationships between climate variables (typically average temperature, but sometimes also humidity or "heating days") and economic variables (potential income, productivity, resource endowments, etc.). It is generally acknowledged that damage functions constitute a weak link in the economics of climate change (Weitzman, 2010).

Various methodologies have been employed for the estimation of their parameters, from subjective expert assessment (Nordhaus, 1994) to panel methods (Dell, Jones and Olken, 2014) to meta-analyses of non-economic literature (Tol, 2002). Also, the functions may be built by summing up different effects into a single aggregate, or they may retain some sectoral detail. The first approach is typical of earlier models like RICE (Nordhaus and Yang, 1996, Nordhaus and Boyer, 1999), MERGE (Manne, Mendelsohn and Richels, 1995) and CETA (Peck and Teisberg, 1992), where a relationship is posited between loss of potential income and temperature. More recent contributions, based on multi-sectoral models like DART (Deke et al., 2001), GTEM (Pant, 2002), ICES (Eboli, Parrado and Roson, 2010) and ENVISAGE (Roson and van der Mensbrugghe, 2012) keep the sectoral detail and attribute the various impacts to different variables and parameters in a disaggregated macroeconomic model, which typically has a Computable General Equilibrium structure.

The main advantage of holding distinct the different economic effects of climate change, despite the cost of higher computational complexity, is that it is possible to trace the various mechanisms through which the climate can affect the economic structure. Furthermore, in a general equilibrium formulation, it is possible to account for second-order effects linked to variations in relative prices, which are often very relevant.

This document illustrates the methodology and presents some results for the estimation of damage functions parameters, for all 140 countries and regions in the GTAP9 dataset, and for six climate impacts: sea level rise, variation in crop yields, heat effects on labor productivity, human health, tourism and household energy demand. Effects from $1^{\circ} \mathrm{C}$ up to $5^{\circ} \mathrm{C}$ average temperature increments are separately considered, as most impacts are non-linear. The work expands and updates some previous estimates by Roson and Sartori (2010), later used for the ENVISAGE model (Roson and van der Mensbrugghe, 2012).

As the GTAP social accounting matrix has become a de-facto standard for the calibration and implementation of computable general equilibrium models, or integrated assessment models with a CGE core, our set of estimates can be seen as a "ready-to-use" information source for the realization of climate-related numerical experiments based on a CGE structure. 
The paper is structured as follows. Sections from 2 to 7 are devoted to presenting the methodology and some estimates for the six impact typologies, whereas detailed numerical results are available in the Appendix at the end of the paper. Section 8 provides a synthesis of the findings by showing first-order approximations of the change in national GDPs triggered by the various effects, when the average temperature is assumed to increase by three Celsius degrees. The results are then discussed in a final concluding section.

\section{Climate change impact \#1: Sea Level Rise}

A large number of studies reviewed by the Fifth IPCC Assessment Report (IPCC, 2014) have shown that the increase in global temperature brings about an increase in the level of the sea. Sea level rise (hereafter SLR) affects the land stock through the erosion, inundation or salt intrusion along the coastline. This phenomenon is in turn generated by (i) the thermal expansion of water bodies and (ii) glaciers' melting.

The share of land which may be lost (in terms of economic production factor) depends on several country-specific characteristics, like: (i) the composition of the shoreline (cliffs and rocky coasts are less subject to erosion than sandy coasts and wetlands); (ii) the total length of the country coast; (iii) the share of the coast which is suitable for productive purposes (i.e. in agriculture); (iv) the vertical land movement (VLM). The latter is a generic term for all processes affecting the elevation at a given location (tectonic movements, subsidence, ground water extraction), causing the land to move up or down. This is typically a slow process with values commonly between $-10 \mathrm{~mm} /$ year (sinking) and +10 $\mathrm{mm} /$ year (rising). Local vertical land movement becomes relevant when looking at the local effects of sea level rise. The orders of magnitude are comparable, and VLM can thus either exacerbate or dampen the SLR.

The literature offers several studies dealing with the SLR, but they are mainly local and country-level studies or macro-level studies, where countries are aggregated into large macro-regions. Perhaps the most employed model is DIVA (Vafeidis et al., 2008), which is an integrated model of coastal systems that assesses biophysical and socio-economic consequences of SLR.

\subsection{Methodology}

The latest IPCC Assessment Report (IPCC AR5) reports the global mean SLR (in meters) associated with the global mean surface temperature change (in ${ }^{\circ} \mathrm{C}$ ), at the time intervals [2046-2065] and [20802100]. These estimates, plotted in Figure 1, suggest that there exist a positive relationship between SLR and the increase in global mean surface temperature, but also a time component, related to the substantial inertia of the physical processes involved.

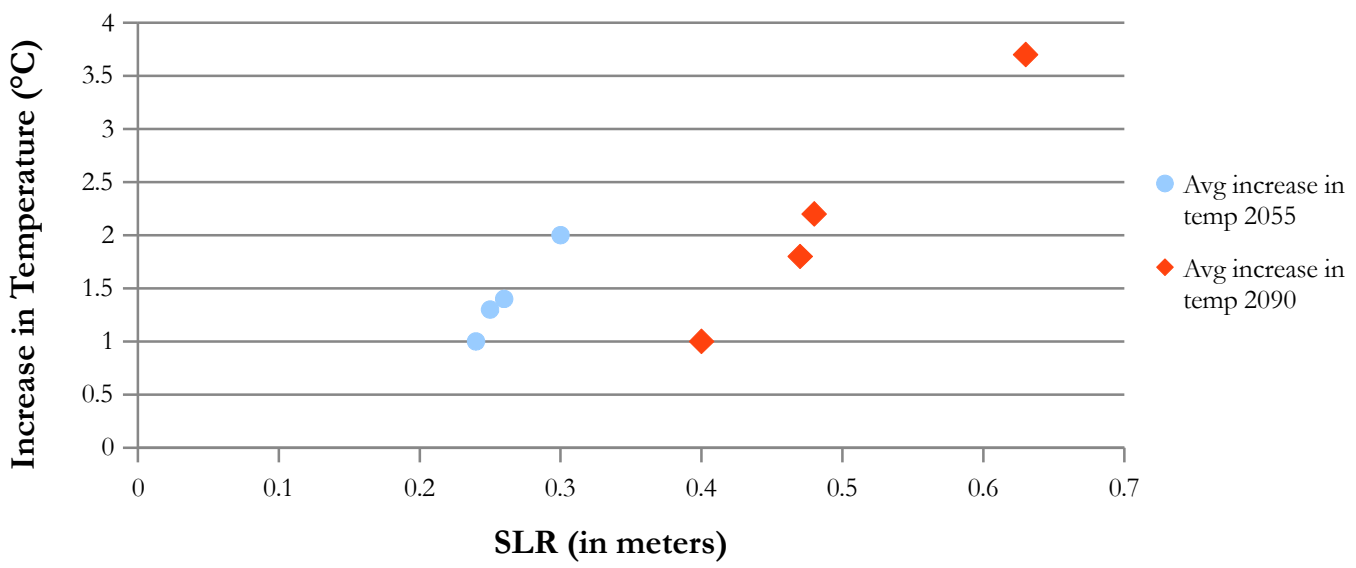

Figure 1. Global mean surface temperature change $\left({ }^{\circ} \mathrm{C}\right)$ and global mean sea level rise (m) 
To better understand the nature of the relationship between the global mean SLR, the increase in the mean global temperature and time, we ran a series of regressions, finding that the following equation provides a satisfactory fit for the relationship:

$$
S L R=[(\alpha+\beta \Delta t)(T-2000)]
$$

where $\Delta t$ is the change in average global temperature with respect to the baseline [1985-2005], and $T$ is the year period. A panel estimation of equation (1) gives a value for the $\alpha$ coefficient of 0.000954281 , whereas the corresponding value for $\beta$ is 0.003421296 .

To account for the vertical land movement $(V)$, equation (1) can be modified as follows:

$$
a S L R=[(\alpha+\beta \Delta t-V)(T-2000)]
$$

where aSLR is the adjusted sea level rise. Data on VLM by country have been retrieved from the SONEL database (www.sonel.org).

For example, the adjusted SLR associated with an increase in temperature of $+1^{\circ} \mathrm{C}$ and VLM of $+0.001 \mathrm{~m} / \mathrm{yr}$ (rising) at the year 2050 is:

$$
0.16878=[(0.000954281+0.003421296 \times 1-0.001)(2050-2000)]
$$

that is, about 0.17 meters.

Using the DIVA v2.04 model, Arnell et al. (2014) provide estimates of the percentage loss in the coastal wetland for 16 macro-regions and 3 single countries. These estimates, reported in Table 1, are associated with a future global mean SLR of $0.16 \mathrm{~m}$, predicted by the HadCM3 climate model under the A1b SRES scenario.

Table 1. \% change in coastal wetland at $0.16 \mathrm{~m}$ of SLR by macro-region (from Arnell et al. 2014)

\begin{tabular}{|c|c|c|c|}
\hline Region/country & $\begin{array}{c}\text { \% change in coastal } \\
\text { wetland by } \mathbf{0 . 1 6} \mathbf{~ m} \\
\text { of SLR }\end{array}$ & Region/country & $\begin{array}{c}\text { \% change in coastal } \\
\text { wetland by 0.16 } \mathbf{~} \\
\text { of SLR }\end{array}$ \\
\hline West Africa & -0.07 & Australasia & -0.12 \\
\hline Central Africa & -0.13 & North Africa & -0.21 \\
\hline East Africa & -0.12 & West Asia & -0.22 \\
\hline South Africa & -0.17 & West Europe & -0.17 \\
\hline South Asia & -0.1 & Central Europe & -0.2 \\
\hline South-East Asia & -0.12 & East Europe & -0.19 \\
\hline East Asia & -0.22 & Canada & -0.06 \\
\hline Central Asia & 0 & USA & -0.24 \\
\hline Meso-America & -0.18 & South America & -0.19 \\
\hline Brazil & -0.09 & - & - \\
\hline
\end{tabular}

Each of the 140 GTAP9 database regions has been associated to one macro-region of Table 1. The percentage loss in coastal wetland (Table 1) has been multiplied by the percentage of erodible coast and 
applied to the whole coast. For the European regions, the shares of erodible coast have been obtained from the Eurosion project (www.eurosion.org), while for the remaining countries we have adopted the $70 \%$ value suggested by Bird $(1987,2010)$. Considering which fraction of total coast is suitable for agricultural and other productive activities we have estimated the fraction of agricultural land which is lost when SLR equals 0.16 meters. Scaling up, we got the share of productive land which is lost for one meter of SLR, labelled LR. Data on coastline length are provided by the CIA database (www.cia.gov); data on the fraction of coast suitable for agricultural activities have been obtained from UNEP (2005).

The percentage change in the land stock by year and country, $L_{R T}$, is computed by multiplying the percentage of effective land change by meter of SLR, LR, and the predicted adjusted SLR, as follows:

$$
L_{R T}=L_{R}\left[\left(\alpha+\beta \Delta t-V_{R}\right)(T-2000)\right]
$$

Notice that the impact function (3) has four parameters. Two parameters $(\alpha, \beta)$ are common across all regions, two other parameters ( $L_{R}$ and $\left.V_{R}\right)$ are country/region specific.

Table A1 in the Appendix shows, for each GTAP9 region, the percentage loss of land by meter of SLR, corresponding to the parameter $L_{R}$ in (3), and the vertical land motion (VLM), corresponding to the parameter $V_{R}$.

Table A2 in the Appendix illustrates the percentage losses of productive land endowments for $+1,+2$, $+3,+4$ and $+5{ }^{\circ} \mathrm{C}$ increases in average temperature, at the years 2050 and 2100 , for all 140 countries and regions. As one can see, relevant physical effects of SLR are concentrated in a few countries, in particular: small island states of Oceania, Central America and Asia, Hong Kong, Japan, Singapore, Jamaica, Puerto Rico, Trinidad and Tobago, Cyprus, Croatia, Bahrain, Kuwait, Qatar, U.A.E. and Mauritius.

\section{Climate change impact \#2: Variation in crop yields (agricultural productivity)}

Climate change is expected to bring about higher temperature, higher concentration of carbon dioxide in the atmosphere, and a different regional pattern of precipitation. These are all factors affecting crop yields and agricultural productivity. Not surprisingly, effects of climate change on agricultural production volumes are perhaps the most studied area of sectoral impacts.

Despite the many studies realized and the extensive empirical evidence produced, however, it is still difficult to identify some sort of "consensus" for the most likely impacts of climate change on agricultural productivity, especially for all world regions. This is because the issue is intrinsically complex and the eventual effect depends on several factors, which are difficult to evaluate ex-ante, for example: (i) the role of adaptation behavior by farmers, firms and organizations, including variety selection, crop rotation, sowing times, etc.; (ii) the amount of fertilization due to higher $\mathrm{CO}_{2}$ concentration; (iii) the actual level of water available for irrigation, and irrigation techniques.

Some studies in this area are based on controlled experiments. Others are based on crop models applied to different crops in different regions and on the basis of different climate scenarios. This heterogenous information is summarized in the latest IPCC Assessment Report (2014), while efforts are under way to standardize the process of agronomic experiments and modeling (AgMIP, 2014).

Because of the heterogeneity of the underlying available information, we follow here two distinct approaches. The first approach, similar to the one adopted by Roson and Sartori (2010), relies on a meta-analysis provided in the Fifth IPCC Assessment Report (2014), providing central estimates for variations in the yields of maize, wheat and rice. We elaborate on these results to get estimates of productivity changes for these three crops, in all 140 regions and for the five levels of temperature increase, from $+1^{\circ} \mathrm{C}$ to $+5^{\circ} \mathrm{C}$. 
The second approach is similar to that of Cline (2007), and brings about an estimate of productivity changes for the whole agricultural sector in the various regions. The decision about which estimates to use in a general equilibrium simulation depends on the level of industrial disaggregation of the model. We suggest to use the first set of parameters if maize, wheat and rice are considered as separate industries, and the second set for the rest, or for the whole agricultural sector if this is regarded as a single aggregate industry.

\subsection{Methodology}

The IPCC AR5, similarly to the previous one, provides a graphical summary (Figure 7-4 in IPCC (2014)) for estimates of changes in productivity of maize, wheat and rice obtained by several studies. It distinguishes between tropical and temperate regions and identify a kind of non-linear interpolation function for the two cases, with and without simple agronomic adaptation. The figure is reproduced here below (Figure 2).
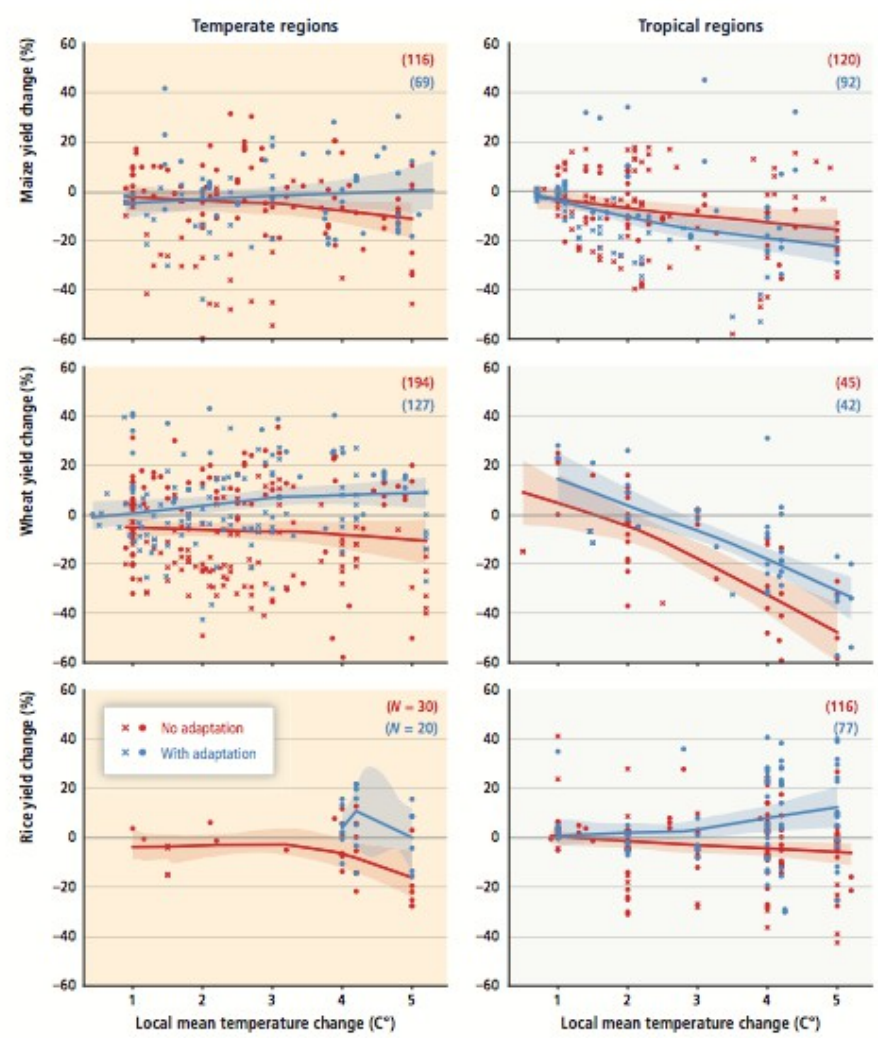

Figure 2. Percentage simulated yield change as a function of local temperature change (from IPCC(2014))

We first express the central values (without adaptation) of Figure 2 as percentage variations in the following table:

Table 2. Central values of the percentage simulated yield change as a function of local temperature change

\begin{tabular}{|l|c|c|c|c|c|c|c|c|c|c|}
\multicolumn{9}{|c}{ Temperate } \\
\hline & $+\mathbf{1}^{\circ} \mathbf{C}$ & $+\mathbf{2}^{\circ} \mathbf{C}$ & $+\mathbf{3}^{\circ} \mathbf{C}$ & $+4^{\circ} \mathbf{C}$ & $+\mathbf{5}^{\circ} \mathbf{C}$ & $+\mathbf{1}^{\circ} \mathbf{C}$ & $+2^{\circ} \mathbf{C}$ & $+3^{\circ} \mathbf{C}$ & $+4^{\circ} \mathbf{C}$ & $+5^{\circ} \mathbf{C}$ \\
\hline Maize & $-1 \%$ & $-3 \%$ & $-4 \%$ & $-7 \%$ & $-11 \%$ & $-4 \%$ & $-8 \%$ & $-10 \%$ & $-12 \%$ & $-14 \%$ \\
\hline Wheat & $-5 \%$ & $-6 \%$ & $-7 \%$ & $-8 \%$ & $-9 \%$ & $4 \%$ & $-4 \%$ & $-20 \%$ & $-34 \%$ & $-44 \%$ \\
\hline Rice & $-4 \%$ & $-3 \%$ & $-2 \%$ & $-7 \%$ & $-16 \%$ & $0 \%$ & $-2 \%$ & $-4 \%$ & $-6 \%$ & $-8 \%$ \\
\hline
\end{tabular}


We then associate the type of region (temperate or tropical) to its latitude, assuming that the reference tropical region has a central latitude of $0^{\circ}$ (the equator) and the reference temperate region has a central latitude of $40^{\circ}$ (North or South). We compute the percentage variation $V Y$ in the yield of crop $C$ in a region with latitude $L$ as:

$$
V Y(C, L)=V Y(C, 0)+(V Y(C, 40)-V Y(C, 0)) * L / 40
$$

Therefore, we assume that the variation in the crop yield ranges linearly from its baseline value at the equator up (or down) to its value at $40^{\circ}$ latitude and beyond. Considering the central latitude of all countries and regions in the GTAP9 dataset, we get the parameters shown in Table A3 of the Appendix.

A second and different methodology is based on the Mendelsohn and Schlesinger (1999) reduced form Agricultural Response Functions in the formulation proposed by Cline (2007), where the variation (DY) in output per hectare is expressed as a function of temperature $T$, precipitation $P$ and $\mathrm{CO} 2$ concentration $K$ :

$$
D Y=115.992 D T-9.936 D T^{2}+0.4752 D P+7.884 D K / K
$$

We need to link changes in yield to variations in average temperature only. To this purpose, we rely on temperature and precipitation data from the USGS Coupled Model Intercomparison Project Phase 5 (CMIP5) Global Climate Change Viewer (GCCV), averaging results from many Global Circulation Models ${ }^{1}$. We collected information on baseline levels and variation in average annual temperature and annual precipitation, by country, comparing the period 1980-2004 (central year 1992) with the period 2050-2074 (central year 2062) under the RCP 8.5 scenario. We also assume that from 1992 to 2062 (70 years) the concentration of CO2 rises (from a baseline level of $365 \mathrm{ppm}$ ) at an annual rate of 2.11 ppm.

We use the variation in temperature as an indicator, expressing how much the climate has changed. By dividing the country-specific variation in precipitation with the one of temperature we get a precipitation to temperature coefficient $p$. In the same way, we get a $\mathrm{CO}_{2}$ concentration to temperature coefficient $k$, so that we can write:

$$
D Y=(115.992+0.4752 p+7.884 k / 365) D T-9.936 D T^{2}
$$

Finally, we need to transform $D Y$ to percentage changes $D Y / Y$, which can be done by dividing $D Y$ by the output per hectare $Y$, in millions of dollars. Cline (2007) uses estimated values for the year 2003 which, unfortunately, vary widely (for example, from 29 in Australia to 8707 in South Korea), ultimately producing unrealistically volatile percentage changes for agricultural productivity.

Here we follow a different strategy, which is based on the "calibration" of the output per hectare $Y$. The latter is chosen so that the percentage change for $+3^{\circ} \mathrm{C}$ is "in line" with a simple mathematical average of estimated variations in the yield of the three crops maize, wheat and rice, for the same temperature change. "In line" means in the range $+/-1 \%$, but conditional on a minimum level for $Y$ of 500 and a maximum level of 10,000 .

1 http://regclim.coas.oregonstate.edu/visualization/gccv/cmip5-global-climate-change-viewer/ 
After calibrating the output per hectare, the percentage variation of agricultural output for 1, 2, 3, 4 and $5^{\circ} \mathrm{C}$ increases in temperature can be computed for each of the 140 GTAP9 countries and regions. The results are shown in Table A4 of the Appendix.

The variation in temperature refers to the average annual temperature specific to each country or region, which may differ from the variation in the global average temperature. On the basis of actual global and regional temperature variations, we estimated for each region a correction factor, which can be used to get an approximated regional variation in temperature through multiplication from the global change. These correction factors are displayed in Table A5 of the Appendix. When only information on the change in global temperature is available, one could therefore estimate the corresponding change in regional temperature using the correction factors.

A quick inspection of the table reveals that variations in regional temperature are typically wider at a higher latitude and whenever the region has limited or no access to the sea or ocean.

\section{Climate change impact \#3: Heat and labor productivity}

Labor productivity is affected by working conditions. Heat stress, determined by high temperature and humidity, implies more frequent pauses, interruptions, lower speed and higher probability of injury (Tawasupa et. al., 2013). Even if acclimatization, on one hand, and protective measures like air conditioning, on the other hand, can help curbing the negative effects of heat stress, the effectiveness and applicability of any adaptation mean is limited and dependent on the context.

Previous work with the ENVISAGE model (Roson and van der Mensbrugghe, 2012), has shown that the impact of increased heat on average labor productivity can be substantial and, furthermore, very much differentiated between developing and developed countries.

To our knowledge, Kjellström et al. (2009) is the only paper investigating the relationship between climate change, heat stress and labor productivity at a global scale. Other works have considered local impacts, or produced regional maps of occupational heat exposure (Hyatt et al., 2010).

In this section we estimate heat damage functions, which are relationships between average temperature and labor productivity. The functions are estimated for three sectors: Agriculture (A), Manufacturing (M) and Services (S) and for 1, 2, 3, 4 and $5{ }^{\circ} \mathrm{C}$ increases in average temperature, bringing about a total of $140 \times 3 \times 5=2100$ estimated parameter values.

\subsection{Methodology}

Most quantitative standards to protect workers from heat injury use the "wet bulb globe temperature" (WBGT) to define the percentage of a typical working hour that a person can work assuming the remaining time is rest. The heat exposure index WBGT (unit $={ }^{\circ} \mathrm{C}$ ) is a combination of the natural wet bulb temperature (measured with a wetted thermometer exposed to the wind and heat radiation at the site), the black globe temperature (measured inside a $150 \mathrm{~mm}$ diameter black globe), and the air temperature (measured with a "normal" thermometer shaded from direct heat radiation). Lemke and Kjellström (2012) propose a methodology to estimate the WBGT from meteorological data.

In this study, following Kjellström et al. (2009), we compute average monthly WBGT using average temperature and relative humidity, on the basis of the Australian Bureau of Meteorology equations:

$$
W B G T=0.567 T+3.94+0.393 E
$$

$$
E=(R H / 100) \times 6.105 \times \exp (17.27 T /(237.7+T))
$$


where $T$ is the average air temperature in ${ }^{\circ} \mathrm{C} ; E$ is the average absolute humidity (water vapour pressure) in $\mathrm{hPa}$; and $\mathrm{RH}$ is the average relative humidity in $\%$.

Monthly average temperature (and precipitation) by country has been obtained from the Weatherbase website (http://www.weatherbase.com/weather/countryall.php3). Unfortunately, data on average relative humidity is not generally available for all countries in our set, but only for specific locations (from http://www.weather-and-climate.com), for example New Delhi (Figure 3).

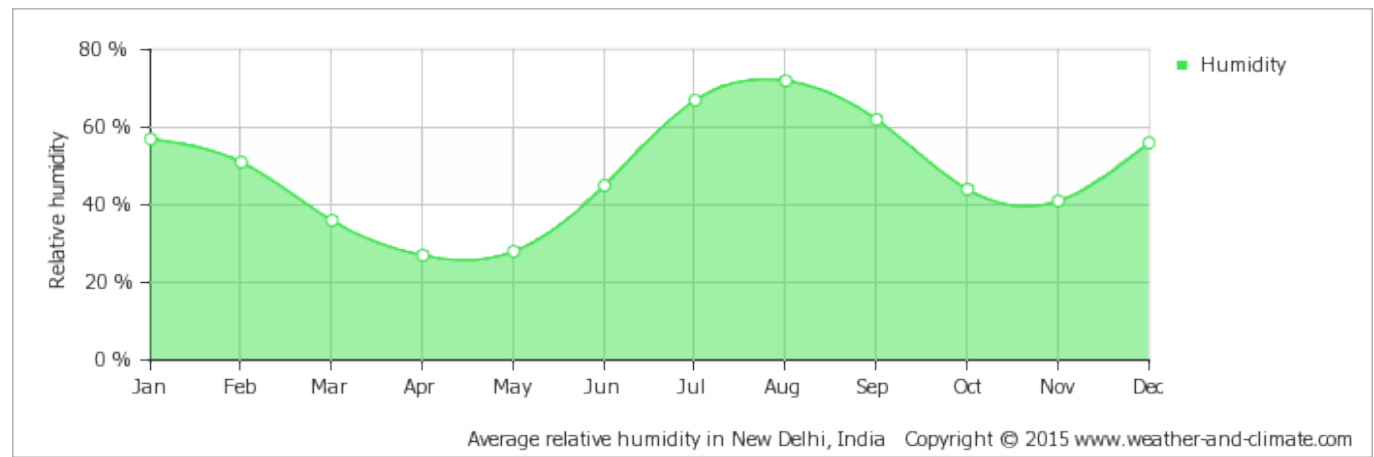

Figure 3. Average relative humidity in New Dehli

In order to approximate the relative humidity from temperature and precipitation data, we ran a series of regressions, finding that the following equation provides a satisfactory estimation:

$$
R H=67.1082-0.8438 T+0.2305 P-0.0005 P^{2}
$$

where $P$ is precipitation in $\mathrm{mm}$.

Therefore, we have computed monthly WBGT for all countries, using temperature and precipitation, in order to assess labor productivity in the three sectors. Kjellström et al. (2009) produced a graph of "work ability" as the maximum percentage of an hour that a worker should be engaged working (Figure 4). The four curves represent four different work intensities. We assume that $200 \mathrm{~W}$ corresponds to office desk work and service industries; $300 \mathrm{~W}$ to average manufacturing industry work and $500 \mathrm{~W}$ to agricultural work.
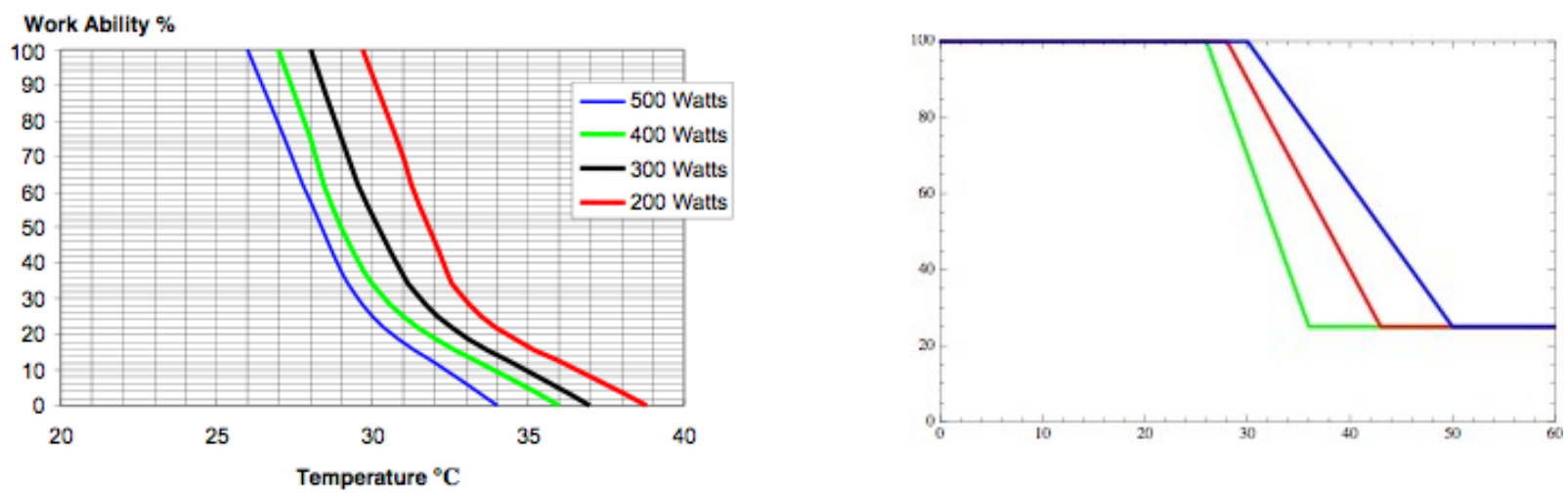

Figure 4. Work ability as a function of WBTG $\left({ }^{\circ} \mathrm{C}\right)$ at four work intensities (Watts), acclimatized (left panel); rescaled (right panel)

We found that curves in Figure 4 (left panel) would give rise to a too rapid and unrealistic decline in productivity at high temperature, especially because we are considering here aggregate averages. We 
have therefore replaced the relationships depicted in the left panel of Figure 4 with the ones shown in the right panel of Figure 4. These are characterized by: (a) a minimum threshold, below which no heat effects are felt $\left(26^{\circ} \mathrm{C}\right.$ for Agriculture, $28^{\circ} \mathrm{C}$ for Manufacturing, $30^{\circ} \mathrm{C}$ for Services), (b) a minimum level of $25 \%$ for productivity, reached at $36^{\circ} \mathrm{C}$ for Agriculture, $43^{\circ} \mathrm{C}$ for Manufacturing and $50^{\circ} \mathrm{C}$ for Services.

We computed the percentage level of productivity for all months, sectors and countries. Monthly values have subsequently been aggregated in a yearly average, since economic flows in many CGE and other numerical models are expressed on an annual basis.

We scaled up temperature levels from 1 to 5 Celsius degrees, assuming that the monthly distribution of temperature will be unaffected and relative humidity stays the same. Finally, we computed the relative percentage change in (annual) productivity with respect to the baseline, for all countries and sectors.

\subsection{Results Overview}

Table A6 in the Appendix presents our estimates for the 140 countries and regions in the GTAP data base. Column headers refer to the sectors $(\mathrm{S}, \mathrm{M}, \mathrm{A})$ and to the increment in temperature $(1,2,3,4$ and $\left.5{ }^{\circ} \mathrm{C}\right)$.

The boxplots in Figure 5 display the distribution of impacts on labor productivity for the three sectors, for the various changes in temperature. In the services, impacts are minimal for a $+1{ }^{\circ} \mathrm{C}$ increase, with a mean of $-0.17 \%$ (maximum impact $-1.67 \%$ in Thailand), but no impacts for 108 out of 140 regions. At five degrees, some effects are felt in about half of the regions (73), with a mean of $-3.71 \%$ and maximum impact $-18.16 \%$ in Singapore. For the manufacturing industries, the effects are more significant, but the distributions are still very much skewed, with 88 regions with no impacts for $+1^{\circ} \mathrm{C}$, 47 for $+5 \%$. The mean percentage variation in labor productivity ranges from $-0.90 \%$ to $-8.12 \%$. The most significant effects are perceived in Singapore, from $-5.96 \%$ to $-31.46 \%$. Agriculture is the sector most significantly affected by higher heat stress. Some effects are felt by about half of the countries (73) already at $+1^{\circ} \mathrm{C}$, but at $+5^{\circ} \mathrm{C}$ only those countries located at sufficiently high latitudes (32) do not experience reductions in labor productivity. The mean percentage variation ranges from $-2.52 \%$ to $-17.48 \%$.
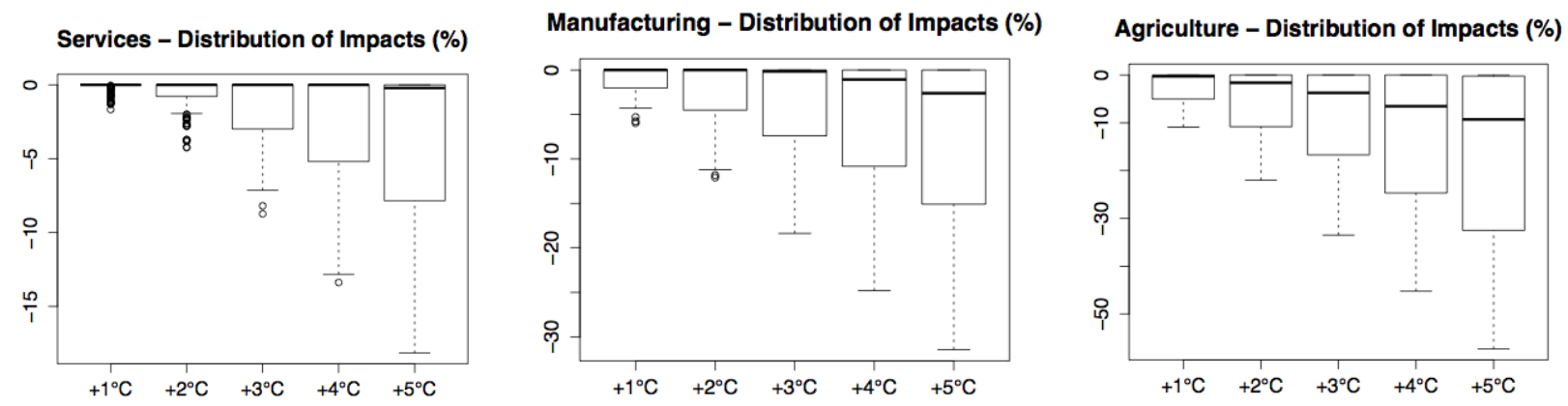

Figure 5. Distribution of impacts on labor productivity in the three sectors, for the various changes in temperature 


\section{Climate change impact \#4: Human Health}

This section describes the methodology and presents some estimates of the effects of increases in temperature on labor productivity, due to changes in mortality and morbidity incidence of some diseases.

Like in Roson and Sartori (2010), the approach follows the one in Bosello, Roson and Tol (2006) by considering some vector-borne diseases (malaria, dengue, schistomiasis), heat and cold related diseases, and diarrhea. It does not consider other diseases and impacts mentioned in the IPCC AR5 (2014), like effects of extreme events, heat exposure effects on labor productivity (separately considered), hemorrhagic fever with renal syndrome, plague, chikungunya fever, japanese and tick-borne encephalitis, cholera and other (non-diarrhea) enteric infections, air quality and nutrition related diseases, allergic diseases, mental health.

Because of lack of data, it is not possible to ascertain possible non-linear impacts of temperature, so the results are expressed as changes in average labor productivity for a $+1^{\circ} \mathrm{C}$ increase in temperature (implicitly assuming that the relationship is approximately linear). Also, the focus is on impacts on labor productivity, whereas other impacts, like those on private and public expenditure for health services, or non-market impacts (e.g., value of life for retired persons) are not taken into account.

We consider only the direct effect of temperature on the incidence of the various diseases, despite the fact that other variables (most notably economic development expressed through income levels) are very important (especially for vector-borne and diarrhea illnesses). To this end, the projected income levels at the year 2050 are taken as reference values for determining the degree of vulnerability in each region. This method implies that indirect effects on human health are not taken into account. For instance, climate change could bring about a reduction of income and a worsening of living conditions, making a society more vulnerable to the direct effects on health.

\subsection{Methodology}

The starting point of the analysis presented in Bosello, Roson and Tol (2006), which is in turn based on Tol (2002), is a survey of the epidemiological, medical and interdisciplinary literature, with the aim of obtaining best estimates for the number of extra cases of mortality and morbidity (for a set of diseases) associated with a given increase in average temperature. These estimates often specify the distribution of cases in the age/sex structure of a population, as well as the length of the illness period (if applicable).

This information can therefore be combined with data on the structure of the working population, to infer the number of lost working days or other variables. For example, Bosello, Roson and Tol (2006), present the following Table 3, expressing the "additional years of life diseased in 2050 by region and disease".

Table 3. Additional years of life diseased in 2050 by region and disease

\begin{tabular}{|l|r|r|r|r|r|r|r|}
\hline & Malaria & \multicolumn{1}{|l}{ Schistom. } & \multicolumn{1}{c|}{ Dengue } & \multicolumn{1}{l}{ Cardio } & Respiratory & Diarrhea & TOT \\
\hline USA & 0 & 0 & 0 & $-167,357$ & 22,257 & 83,070 & $-62,030$ \\
\hline Europe Un. & 0 & 0 & 0 & $-171,908$ & 20,936 & 25,608 & $-125,364$ \\
\hline E.E.F.S.U. & 0 & 0 & 0 & $-259,884$ & 46,884 & 57,717 & $-155,283$ \\
\hline Japan & 0 & 0 & 0 & $-65,353$ & 33,161 & 912 & $-31,280$ \\
\hline Rest Ann.I & 0 & 0 & 0 & $-45,232$ & 11,108 & 1,361 & $-32,763$ \\
\hline Energy Exp. & 7,219 & $-1,088$ & 29 & $-66,363$ & $1,706,267$ & 112,633 & $1,758,697$ \\
\hline China India & 632 & 0 & 0 & $-1,119,902$ & 770,340 & 156,271 & $-192,659$ \\
\hline Rest World & 232,737 & $-154,375$ & 203 & $-194,383$ & $3,683,042$ & 834,294 & $44,01,518$ \\
\hline
\end{tabular}


In this study, we review the most recent literature on health impacts, and in particular some studies mentioned in IPCC (2014), to modify the figures contained in Table 3 above, with the aim of scaling up or down the variation in labor productivity calculated by Roson and Sartori (2010). For example, the change in labor productivity assumed for Japan, for $+1{ }^{\circ} \mathrm{C}$, was $+0.034 \%$, which corresponds to the -31280 decrease in diseased years in Table 3. Our updated estimates for the number of diseased years in Japan point to an increase in the number of years (+57894), corresponding to a change in labor productivity of $-0.063 \%$.

The procedure is slightly more complicated if several countries are included in the same macro-region, especially if those estimates of changes in productivity showed in Roson and Sartori (2010) have different sign. In this case, the original estimates are still multiplied by a correction factor, but the magnitude of the factor is determined by a mathematical optimization software, ensuring that the average variation in productivity for the whole group is consistent with the updated figures of diseased years.

For malaria, our primary source is Béguin et al. (2011), who suggest that extra cases of malaria, net of the effect due to income growth, should only be found in Africa and China/India. Correspondingly, we set to zero the impact for Energy Exporting Countries, while increasing by $1 / 3$ the number of cases (diseased years) in Africa and China/India.

For schistomiasis, it is unclear why in the original estimates by Tol (2002) an increase in temperature should produce a decrease in the number of cases, if the effect of temperature is considered net of the impact of higher income levels. Actually, some studies highlight that climate change is expected to create the conditions for a potential spreading of the disease in some regions, for example in China (Zhou et al., 2008). Therefore, we decide to disregard any impact for schistomiasis, by putting zeros in the corresponding column.

Dengue is the most rapidly spreading mosquito-borne viral disease, showing a 30-fold increase in global incidence over the past 50 years (WHO, 2013). However, according to Åström et al. (2012) the geographic distribution of dengue is strongly dependent on both climatic and socioeconomic variables. They present a model showing that, under a scenario of constant per capita GDP, global climate change results in a modest but important increase in the global population at risk of dengue. Under scenarios of high GDP growth, this adverse effect of climate change is counteracted by the beneficial effect of socioeconomic development. With higher income sets at projected 2050 levels, the vulnerability to dengue fever is rather low. We accommodate for this information by concentrating all extra cases of dengue in Africa, and by setting the figures of diseased years at $10 \%$ of their original levels in the benchmark Table 3.

Among heat-related illnesses we consider, in line with Tol (2002), respiratory and a share of cardiovascular diseases. As the recent literature on heat risks for health (e.g., Honda et al. 2013) does not present very significant changes from earlier estimates, the contribution of heat-related diseases to the overall variation in labor productivity has been kept unchanged. The same reasoning applies to health impacts of changes in diarrhea cases (Kolstad and Johansson, 2011).

On the contrary, our assumptions about cold-related diseases are dramatically different. In Bosello, Roson and Tol (2006), consistently with Table 3, a reduction of cold-related cases brings about a reduction of mortality/morbidity in most countries, and an increase in labor productivity. However, the recent epidemiological literature has questioned the finding of a positive effect of higher temperature levels on winter mortality and morbidity. For example, Ebi and Mills (2013) argue that although there is a physiological basis for increased cardiovascular and respiratory disease mortality during winter months, the limited evidence suggests cardiovascular disease mortality is only weakly associated with temperature. This is because several illnesses have a strong seasonal component, in which relative temperature, not absolute temperature, actually matters. Correspondingly, we disregard any effect of climate change on cold-related diseases. This has very important implications for our estimates, because now all health impacts become negative in all countries. 


\subsection{Results Overview}

The estimated percentage variation of labor productivity for 140 regions and for a $+1^{\circ} \mathrm{C}$ increase in temperature is presented in Table A7 of the Appendix. The unweighed average is $-0.27 \%$, and the range is from $-0.75 \%$ (India, Nepal and Sri Lanka) to 0\% (Canada).

The variations can be grouped in 32 classes. Figure 6 displays the number of countries in each class. The three most numerous classes are: $-0.631 \%$ (African countries), $-0.034 \%$ (Western Europe), $-0.135 \%$ (Central America).

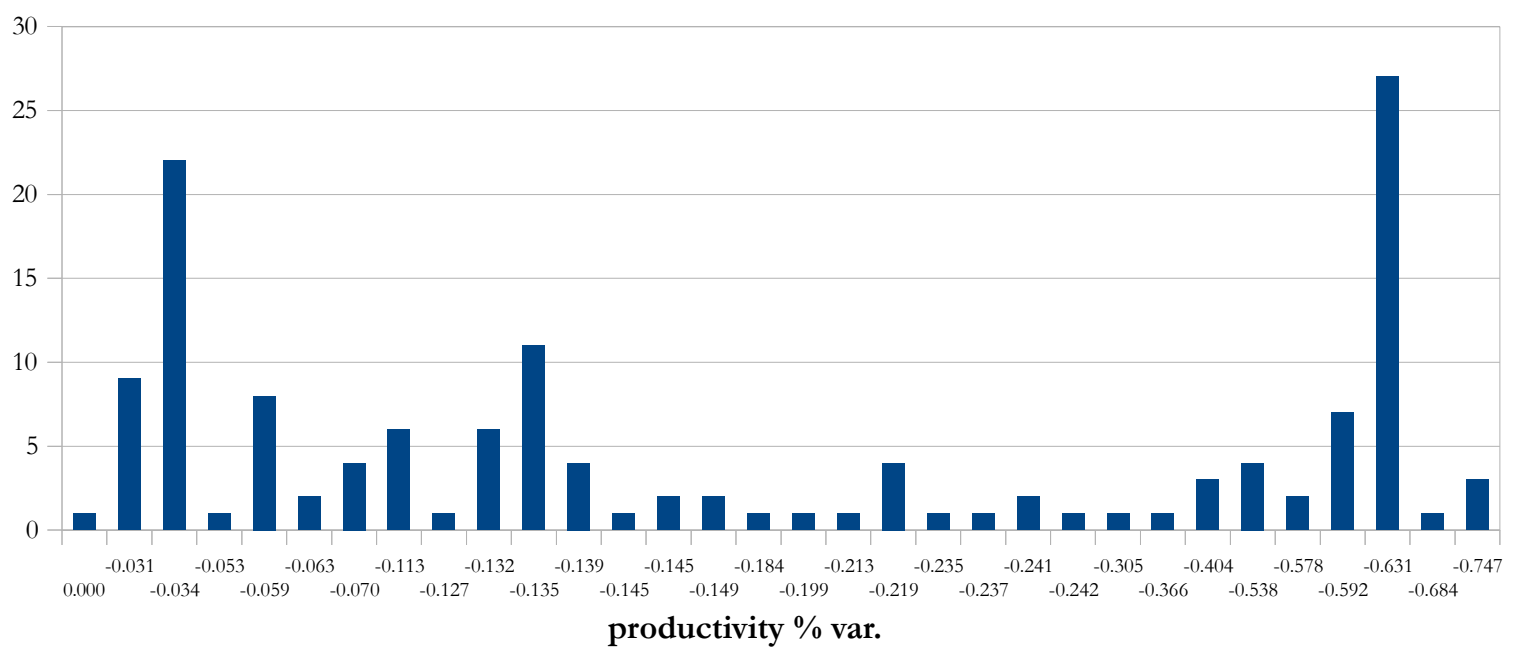

Figure 6. Number of countries in each class

\section{Climate change impact \#5: Tourism}

Climate is one of the main drivers of international tourism, and tourism revenue is a fundamental pillar of the economy in many countries. It is surprising that the tourism literature pays little attention to climate and climatic change and, when it does so, the analysis is typically based on local case studies.

It is equally surprising that the climate change impact literature pays little attention to tourism. Previous work with the ENVISAGE model (Roson and van der Mensbrugghe, 2012) has shown that the impact of changing tourism attractiveness can be substantial, bringing about a sizable redistribution of income among various countries.

Perhaps the only study conducting a quantitative assessment of climate impacts on international tourism flows, at a global scale, is Hamilton, Maddison and Tol (2005). We start from some functions and parameters computed in this study to elaborate data on arrivals, departures, temperature and expenditure. The ultimate goal is estimating a relationship between average temperature changes and net inflow of foreign currency and expenditure of foreign tourists in the hosting country.

\subsection{Methodology}

Hamilton, Maddison and Tol (2005) have built an econometric model for the estimation of international tourism flows. They used econometric techniques to estimate parameters of two functions. In the first function, the logarithm of yearly arrivals of tourists in a country is expressed as a function of land area, average temperature, length of coastline and per capita income. In a second function, the logarithm of the ratio of departures over population is expressed as a function of temperature, income, land area and number of countries with shared land borders. 
We take these two functional relationships to get equations linking arrivals $(A)$ and departures $(D)$ in a region solely to its average temperature $(T)$, in Celsius degrees:

$$
\begin{aligned}
& A=K_{A} \times \exp \left(0.22 T-0.00791 T^{2}\right) \\
& D=K_{D} \times \exp \left(-0.18 T+0.00438 T^{2}\right)
\end{aligned}
$$

where $K_{A}$ and $K_{D}$ are country-specific constants, accounting for all other factors different from temperature. We calibrate these parameters on the basis of regional data on yearly arrivals, departures and average temperature.

We can see that both relationships are non-linear. The maximum number of arrivals is obtained at the optimal average temperature of $13.9^{\circ} \mathrm{C}$. The minimum number of departures is obtained at $18.6^{\circ} \mathrm{C}$. For increases in temperature below the $13.9^{\circ} \mathrm{C}$ threshold, arrivals increase and departures decrease, therefore a country gets a beneficial net inflow of foreign currency. The opposite is found for increases in temperature above the $18.6^{\circ} \mathrm{C}$ threshold. For variations between $13.9^{\circ} \mathrm{C}$ and $18.6^{\circ} \mathrm{C}$, effects are ambiguous, not only because arrivals and departures push to different directions, but also because the average expenditure level of an incoming tourist may be different from the expenditure level of an outgoing tourist ${ }^{2}$.

We estimated changes in arrivals and departures for $1,2,3,4$ and $5{ }^{\circ} \mathrm{C}$ increases in average temperature from its baseline level, for all 140 countries and regions. Variations in arrivals multiplied by per capita expenditure minus variations in departures multiplied by per capita expenditure give a first estimate of changes in net foreign currency inflow.

Of course, changes can be be both positive and negative. Furthermore, summing up all changes does not typically gives a zero result. However, as it will be made clearer in Sub-section 6.3, if foreign currency flows are interpreted as international income transfers, we would actually need to impose that all variations sum up to one.

To this end, we scaled up or down all our estimates, by subtracting the average net inflow if positive, or adding it if it turns out to be negative. One possible interpretation of this ex-post rescaling is in terms of relative competitiveness, since flows are not only affected by local conditions, but also by conditions in competing destinations.

\subsection{Results overview}

Our rescaled estimates of changes in net foreign currency inflows, relative to the 2011 GDP level, are displayed in Table A8 of the Appendix. These variations follows a rather non-linear path. Limited increases of temperature are beneficial but higher levels are detrimental in China, South Korea, Italy and Turkey. Vice versa, initial negative impacts turn positive at $+5^{\circ} \mathrm{C}$ in Mongolia, Estonia, Lithuania, Slovakia, Slovenia, Bulgaria, Belarus, Romania and Kazakhstan.

Benefits are concentrated in a few countries. For example, at $+3{ }^{\circ} \mathrm{C}$ only 26 countries get an increase in tourism revenue, whereas as many as 97 countries experience a relative loss. Benefitted countries include North European and North American countries, Japan and Russia, which are all rich nations: tourism impacts have adverse distributional consequences.

Furthermore, the dispersion of income flows gets larger as temperature rises. The standard deviation of the distribution of net revenue inflows increases progressively from about 1.48 billions US $\$$ at $+1{ }^{\circ} \mathrm{C}$ up to around 5.36 billions US\$ at $+5^{\circ} \mathrm{C}$.

2 We estimated per capita expenditure data on the basis of IMF data on tourism revenue (IMF, 2014). 


\subsection{Inclusion of Tourism Impacts in a CGE Model}

Our estimates of net currency inflows are meant to be used as inputs in a CGE model, assessing economic impacts of climate change. The exogenous shock can be inserted as a variation in international income transfers and, possibly, as a shift in the pattern of final consumption.

Most CGE models are based on a "territorial" definition of income. In other words, GDP rather than GNP is taken as the reference value for income and other macroeconomic variables. This implies that there is no distinction between nationals and foreigners when income is spent inside a country boundaries. However, the purchasing power of foreigners comes from income generated abroad. In order to consider this important aspect, Berrittella et al. (2006) and Bigano et al. (2008) simulate the occurrence of some international income transfers, whose magnitude corresponds to the estimated change in net currency inflows.

Since foreign tourists are unlikely to have a structure of consumption similar to that of the representative household in a country, a further step is simulating an exogenous increase (or decrease) in the consumption of tourism (hotels, restaurants, recreation facilities) and domestic transport services, which can be implemented by inserting some shifting parameters in the final demand for these items.

\section{Climate change impact \#6: Household Energy Demand}

Household energy demand is directly affected by variations in temperature. This relationship is rather complex, as the impact on energy consumption depends on the season, the source of energy and the climatic condition of the country.

For instance, an increase in winter temperatures would cause a decrease in energy used for heating purposes, whereas an increase in summer temperatures is likely to cause an increase of energy consumed for cooling purposes, depending on the latitude of the country (i.e., tropical, temperate, cold).

In what follows, the impact of increasing average temperature on energy demand is computed, taking into account all these factors.

\subsection{Methodology}

Our estimates are based on De Cian, Lanzi and Roson (2013), who computed parameters of a model for household energy demand, by energy source and season, using econometric techniques and a global panel database. Energy demand is expressed as dependent, among other factors, on the (natural logarithm of) seasonal average temperature, expressed in ${ }^{\circ} \mathrm{F}$.

Seasonal long run temperature elasticities by energy source and by climate region (Table 4) are those estimated by De Cian, Lanzi and Roson (2013.). Since we are interested in the variation of total energy demand, elasticities in Table 4 have been scaled down by considering the share of energy used for heating and cooling purposes (Table 5). The adjusted elasticities are shown in Table 6.

Data on average seasonal temperature by country are obtained from the Weather Database (www.weatherbase.com), whereas each country has been classified as Cold, Mild or Hot, according to its latitude. ${ }^{3}$ Applying the model estimated by De Cian, Lanzi and Roson (2013), to the percentage variation in temperature corresponding to $1^{\circ} \mathrm{C}$ (and $2,3,4,5^{\circ} \mathrm{C}$ ) increase in seasonal average temperature has been multiplied by the elasticities reported in Table 6 .

\subsection{Result overview}

3 Hot countries: latitude $<27^{\circ}$; mild countries: $27^{\circ}<$ latitude $<63^{\circ}$; cold countries: latitude $>63^{\circ}$. For aggregated regions the latitude has been computed as a weighted sum of the latitude of each single country. 
Table A9 in the Appendix shows our estimates of the percentage variations in household energy demand corresponding to a $+1,+2,+3,+4$ and $+5^{\circ} \mathrm{C}$ increase in the average seasonal temperature. Estimates are provided for the 140 GTAP9 regions, but they are available for more countries.

A quick inspection of the table reveals that: (i) household demand for electricity rises, especially in the hot countries, as this source of energy is mainly used for air conditioning. The highest relative growth is expected in the African countries; (ii) household demand for energy from oil products dramatically decreases in all countries, especially in cold countries; (iii) the effect on household demand for energy from gas is positive (negative) in mild and cold (hot) countries.

Table 4. Long run temperature elasticities from De Cian et al. (2013)

\begin{tabular}{|c|l|c|c|c|}
\hline Season & Climate & Electr. & Gas & Oil.P. \\
\hline Winter & Cold & -0.085 & -0.422 & -0.406 \\
& Mild & -0.085 & -0.422 & -0.406 \\
& Hot & -0.085 & -0.422 & -0.406 \\
\hline Spring & Cold & 0.522 & 0.686 & -0.395 \\
& Mild & -0.077 & 0.686 & -0.395 \\
& Hot & 0.263 & 0.686 & -0.395 \\
\hline Summer & Cold & -0.321 & -1.008 & -0.912 \\
& Mild & 0.2 & -1.008 & -0.912 \\
& Hot & 0.174 & -1.008 & -0.912 \\
\hline \multirow{2}{*}{ Fall Cold } & - & 0.685 & 0.0002 \\
& Mild & - & 0.685 & 0.0002 \\
& Hot & - & 0.685 & 0.0002 \\
\hline
\end{tabular}

Table 5. Share of energy demanded for heating and cooling purposes, by energy source and climate region.

Source: U.S. Residential Energy Demand Database (www.eia.gov)

\begin{tabular}{|r|c|c|c|c|}
\cline { 2 - 5 } \multicolumn{1}{c|}{} & \multicolumn{2}{|c|}{ Electricity } & Gas & Oil P. \\
\hline Climate & Heating & Cooling & Heating & Heating \\
\hline Cold & $8 \%$ & $5 \%$ & $72 \%$ & $88 \%$ \\
Mild & $9 \%$ & $17 \%$ & $56 \%$ & $86 \%$ \\
Hot & $7 \%$ & $28 \%$ & $48 \%$ & $86 \%$ \\
\hline
\end{tabular}


Table 6. Adjusted long run temperature elasticities

\begin{tabular}{|c|l|c|c|c|}
\hline Season & Climate & Electr. & Gas & Oil.P. \\
\hline Winter & Cold & -0.0111 & -0.3053 & -0.3558 \\
& Mild & -0.0221 & -0.2345 & -0.3496 \\
& Hot & -0.0300 & -0.2008 & -0.3496 \\
\hline Spring & Cold & 0.0682 & 0.4962 & -0.3462 \\
& Mild & -0.0200 & 0.3812 & -0.3401 \\
& Hot & 0.0929 & 0.3264 & -0.3401 \\
\hline Summer & Cold & -0.0419 & -0.7292 & -0.7993 \\
& Mild & 0.0519 & -0.5602 & -0.7853 \\
& Hot & 0.0614 & -0.4797 & -0.7853 \\
\hline \multicolumn{2}{|c|}{ Fall Cold } & - & 0.4955 & 0.0002 \\
& Mild & - & 0.3807 & 0.0002 \\
& Hot & - & 0.3260 & 0.0002 \\
\hline
\end{tabular}

\section{Aggregation of impacts and first-order effects on GDP}

The illustration of our estimates for the different impacts of the climate change has made clear that the impacts are different in sign, magnitude and relevance for the various countries and regions. Therefore, it would be interesting to see what is the net aggregate effect, for example in terms of real income or GDP, of the combined impacts.

A full fledged analysis of this kind would require a global, disaggregated macroeconomic model, in which our estimates would be employed to shock exogenous parameters. For instance, an exogenous reduction in agricultural productivity would reduce the relative competitiveness for the domestic agricultural sector, increasing imports from abroad, inducing a real devaluation, expanding production and exports in manufacturing and services.

Such kind of analysis is beyond the scope of this paper. Nonetheless, we can provide here a first-order approximation of the impact on the real GDP, because most of the impacts affect variables which are components of the Gross Domestic Product, with the exception of the variation in energy demand. Because of that, an approximated impact on the GDP can be readily obtained by multiplying the variation of one GDP component by its share, and in particular:

- impacts of sea level rise on GDP can be gauged by multiplying the estimated changes in available land resources by the share of land rents income on total GDP;

- agricultural productivity variations can be evaluated by multiplying the changes by the share of agricultural value added on total GDP;

- the reduction in labor productivity due to heat stress has an effect on the GDP that can be estimated as the sum of variations in labor productivity in the three sectors (agriculture, manufacturing, services) multiplied by the shares of (sectoral) labor income on total GDP;

- human health effects can be obtained by multiplying the estimated changes by the share of labor income on total GDP;

- the net inflow of foreign currency due to tourism flows can be directly expressed as relative to a baseline GDP level.

Even if the sum of the different impacts on GDP is only limited to first-order effects and does not consider general equilibrium feedbacks, we believe that such an approximation of the composite GDP footprint could reveal important insights about the order of magnitude, relevance, and distribution of 
the various impacts. Tables 7-1 and 7-2 present our estimates, corresponding to an increase in average temperature of $+3^{\circ} \mathrm{C}^{4}$ for the five categories above and their total algebraic sum. We highlight with a green background color the positive net variations in GDP, with a yellow background moderate reductions (from $-1 \%$ to $-5 \%$ ) and with a red background the large reductions (below $-5 \%$ ). In addition, we identify, for each country, which among the three types of impact is the one which contributes the most to the overall effect on GDP. ${ }^{5}$

A quick inspection of Tables 7-1 and 7-2 reveals a number of thought-provoking facts. Only a few countries (Mongolia, Canada, and central-northern European countries, including Russia) are expected to get moderate gains from a $+3^{\circ} \mathrm{C}$ increase in temperature, and these gains are typically due to an increase in tourists' arrivals (and diminished outgoing domestic tourists). Many countries (whose estimates are highlighted in red) are expected to suffer from dramatic reductions in GDP. The most negatively affected countries are Togo in Africa (-18.29\%) and Cambodia in South-East Asia (-18.25\%), where again Tourism is the most important factor.

In addition to tourism income, variations in agricultural and labor productivity are also very relevant in many countries. Sea level rise, on the other hand, never appears as the primary factor, because of its limited incidence on total land and the relative small share of land income on GDP. Remarkably, Tourism is (possibly with Heat) the least studied effect of climate change, maybe because it causes a redistribution of income and wealth, but it has negligible consequences at the global level.

4 This refers to changes in the global average temperature. For agricultural productivity, we consider regional variations, which could be larger or smaller than the global one. Furthermore, sea level rise does not depend only on temperature levels, but on time. For this estimation, we set the year 2100 as the one corresponding to the $+3^{\circ} \mathrm{C}$ temperature increment. 5 Therefore, it has the same sign of the total variation. 
Table 7-1. Impact on GDP of $+3^{\circ} \mathrm{C}$ by country

\begin{tabular}{|c|c|c|c|c|c|c|c|}
\hline Code & SLR & AGR & HEAT & HEALTH & TOURISM & $\begin{array}{l}\text { Incidence on } \\
\text { GDP of }+3^{\circ} \mathrm{C}\end{array}$ & $\begin{array}{c}\text { Dominant } \\
\text { impact }\end{array}$ \\
\hline \begin{tabular}{l|l}
1 & AUS
\end{tabular} & $0.0000 \%$ & $-0.1686 \%$ & $-0.0162 \%$ & $-0.2370 \%$ & $-0.5029 \%$ & $-0.92 \%$ & TOURISM \\
\hline $2 \mathrm{NZL}$ & $-0.0005 \%$ & $-0.0975 \%$ & $0.0000 \%$ & $-0.2073 \%$ & $0.1806 \%$ & $-0.12 \%$ & HEALTH \\
\hline $3 \times O C$ & $-0.0095 \%$ & $-0.3135 \%$ & $-1.3971 \%$ & $-0.3030 \%$ & $0.0000 \%$ & $-2.02 \%$ & HEAT \\
\hline $4 \mathrm{CHN}$ & $0.0000 \%$ & $0.1975 \%$ & $-0.5449 \%$ & $-0.8164 \%$ & $0.0890 \%$ & $-1.07 \%$ & HEALTH \\
\hline 5 HKG & $-0.0118 \%$ & $-0.0480 \%$ & $-1.6329 \%$ & $-0.7237 \%$ & $-5.2541 \%$ & $-7.67 \%$ & TOURISM \\
\hline 6 JPN & $-0.0005 \%$ & $-0.0765 \%$ & $-0.2334 \%$ & $-0.0967 \%$ & $0.0205 \%$ & $-0.39 \%$ & HEAT \\
\hline 7 KOR & $-0.0006 \%$ & $-0.1113 \%$ & $-0.2600 \%$ & $-0.0843 \%$ & $0.2123 \%$ & $-0.24 \%$ & HEAT \\
\hline 8 MNG & $0.0000 \%$ & $0.5520 \%$ & $0.0000 \%$ & $-0.4409 \%$ & $0.9466 \%$ & $1.06 \%$ & TOURISM \\
\hline 9TWN & $-0.0004 \%$ & $-0.1019 \%$ & $-2.4258 \%$ & $-0.9099 \%$ & $-2.0929 \%$ & $-5.53 \%$ & HEAT \\
\hline 10 XEA & $-0.0010 \%$ & $-0.3961 \%$ & $-4.2472 \%$ & $-0.1915 \%$ & $0.0000 \%$ & $-4.84 \%$ & HEAT \\
\hline 11 BRN & $-0.0001 \%$ & $-0.0059 \%$ & $-2.0021 \%$ & $-0.1206 \%$ & $-2.6786 \%$ & $-4.81 \%$ & TOURISM \\
\hline $12 \mathrm{KHM}$ & $-0.0002 \%$ & $-2.1774 \%$ & $-5.2924 \%$ & $-0.1315 \%$ & $-10.6492 \%$ & $-18.25 \%$ & TOURISM \\
\hline 13 IDN & $-0.0010 \%$ & $-1.1587 \%$ & $-4.7511 \%$ & $-0.1790 \%$ & $-0.7110 \%$ & $-6.80 \%$ & HEAT \\
\hline 14 LAO & $0.0000 \%$ & $-3.5049 \%$ & $-4.1597 \%$ & $-0.1425 \%$ & $-5.7644 \%$ & $-13.57 \%$ & TOURISM \\
\hline 15 MYS & $-0.0005 \%$ & $-0.7494 \%$ & $-4.8378 \%$ & $-0.1816 \%$ & $-4.4406 \%$ & $-10.21 \%$ & HEAT \\
\hline 16 PHL & $-0.0028 \%$ & $-0.9965 \%$ & $-4.6830 \%$ & $-0.1445 \%$ & $-1.5898 \%$ & $-7.42 \%$ & HEAT \\
\hline 17 SGP & $-0.0020 \%$ & $-0.0200 \%$ & $-4.4945 \%$ & $-0.2987 \%$ & $-5.9202 \%$ & $-10.74 \%$ & TOURISM \\
\hline 18 THA & $-0.0001 \%$ & $-0.7803 \%$ & $-3.7029 \%$ & $-0.1419 \%$ & $-4.5046 \%$ & $-9.13 \%$ & TOURISM \\
\hline 19VNM & $-0.0006 \%$ & $-1.3580 \%$ & $-3.3932 \%$ & $-0.1501 \%$ & $-2.1889 \%$ & $-7.09 \%$ & HEAT \\
\hline 20 XSE & $-0.0010 \%$ & $-3.2015 \%$ & $-6.4740 \%$ & $-0.1549 \%$ & $0.0000 \%$ & $-9.83 \%$ & HEAT \\
\hline $21 \mathrm{BGD}$ & $-0.0001 \%$ & $-1.2004 \%$ & $-3.2480 \%$ & $-0.2020 \%$ & $-0.3383 \%$ & $-4.99 \%$ & HEAT \\
\hline 22 IND & $-0.0001 \%$ & $-1.3077 \%$ & $-3.3046 \%$ & $-1.0484 \%$ & $-0.5829 \%$ & $-6.24 \%$ & HEAT \\
\hline $23 \mathrm{NPL}$ & $0.0000 \%$ & $-0.0773 \%$ & $-1.1111 \%$ & $-0.9108 \%$ & $-1.8753 \%$ & $-3.97 \%$ & TOURISM \\
\hline 24 PAK & $0.0000 \%$ & $-1.7497 \%$ & $-1.2167 \%$ & $-0.0985 \%$ & $-0.2498 \%$ & $-3.31 \%$ & AGR \\
\hline 25 LKA & $-0.0008 \%$ & $-1.3164 \%$ & $-2.9340 \%$ & $-0.8583 \%$ & $-1.2886 \%$ & $-6.40 \%$ & HEAT \\
\hline $26 \times S A$ & $0.0000 \%$ & $-1.9427 \%$ & $-2.8045 \%$ & $-0.1434 \%$ & $0.0000 \%$ & $-4.89 \%$ & HEAT \\
\hline 27 CAN & $-0.0001 \%$ & $0.1723 \%$ & $0.0000 \%$ & $0.0000 \%$ & $1.1003 \%$ & $1.27 \%$ & TOURISM \\
\hline 28 USA & $0.0000 \%$ & $0.0159 \%$ & $-0.0048 \%$ & $-0.2896 \%$ & $0.1152 \%$ & $-0.16 \%$ & HEALTH \\
\hline 29 MEX & $0.0000 \%$ & $-0.3420 \%$ & $-0.1530 \%$ & $-0.2326 \%$ & $-0.4177 \%$ & $-1.15 \%$ & TOURISM \\
\hline 30 XNA & $-0.0033 \%$ & $0.0118 \%$ & $-0.0037 \%$ & $-0.3277 \%$ & $0.0000 \%$ & $-0.32 \%$ & HEALTH \\
\hline 31 ARG & $0.0000 \%$ & $-0.2384 \%$ & $-0.1037 \%$ & $-0.3114 \%$ & $-0.2509 \%$ & $-0.90 \%$ & HEALTH \\
\hline $32 \mathrm{BOL}$ & $0.0000 \%$ & $-1.3641 \%$ & $0.0000 \%$ & $-0.1476 \%$ & $-1.3293 \%$ & $-2.84 \%$ & AGR \\
\hline 33 BRA & $0.0000 \%$ & $-0.5921 \%$ & $-0.8644 \%$ & $-0.3432 \%$ & $-0.3293 \%$ & $-2.13 \%$ & HEAT \\
\hline $34 \mathrm{CHL}$ & $-0.0002 \%$ & $0.0103 \%$ & $0.0000 \%$ & $-0.2737 \%$ & $0.0007 \%$ & $-0.26 \%$ & HEALTH \\
\hline $35 \mathrm{COL}$ & $-0.0001 \%$ & $-0.7781 \%$ & $-0.9717 \%$ & $-0.1258 \%$ & $-0.6461 \%$ & $-2.52 \%$ & HEAT \\
\hline 36 ECU & $-0.0004 \%$ & $-1.0763 \%$ & $0.0000 \%$ & $-0.1526 \%$ & $-0.7002 \%$ & $-1.93 \%$ & AGR \\
\hline 37 PRY & $0.0000 \%$ & $-1.9012 \%$ & $-2.2562 \%$ & $-0.1768 \%$ & $-1.4291 \%$ & $-5.76 \%$ & HEAT \\
\hline 38 PER & $-0.0002 \%$ & $-1.4078 \%$ & $0.0000 \%$ & $-0.1868 \%$ & $-0.3127 \%$ & $-1.91 \%$ & AGR \\
\hline 39 URY & $-0.0001 \%$ & $-0.4524 \%$ & $-0.0572 \%$ & $-0.2972 \%$ & $-1.3583 \%$ & $-2.17 \%$ & TOURISM \\
\hline 40 VEN & $-0.0001 \%$ & $-0.6564 \%$ & $-0.9783 \%$ & $-0.1686 \%$ & $-0.3473 \%$ & $-2.15 \%$ & HEAT \\
\hline 41 XSM & $-0.0013 \%$ & $-0.4069 \%$ & $-0.0462 \%$ & $-0.1470 \%$ & $0.0000 \%$ & $-0.60 \%$ & AGR \\
\hline 42 CRI & $-0.0011 \%$ & $-0.8385 \%$ & $-1.9108 \%$ & $-0.2989 \%$ & $-3.1429 \%$ & $-6.19 \%$ & TOURISM \\
\hline 43 GTM & $-0.0002 \%$ & $-1.4468 \%$ & $-0.3188 \%$ & $-0.1860 \%$ & $-1.6208 \%$ & $-3.57 \%$ & TOURISM \\
\hline 44 HND & $-0.0005 \%$ & $-1.3208 \%$ & $-4.0728 \%$ & $-0.1931 \%$ & $-3.5740 \%$ & $-9.16 \%$ & HEAT \\
\hline 45 NIC & $-0.0006 \%$ & $-1.8717 \%$ & $-5.0354 \%$ & $-0.1958 \%$ & $-5.0277 \%$ & $-12.13 \%$ & HEAT \\
\hline 46 SLV & $-0.0002 \%$ & $-0.6504 \%$ & $-2.7781 \%$ & $-0.1926 \%$ & $-1.4962 \%$ & $-5.12 \%$ & HEAT \\
\hline 47 PAN & $-0.0019 \%$ & $-1.2835 \%$ & $-0.9629 \%$ & $-0.1481 \%$ & $-7.1071 \%$ & $-9.50 \%$ & TOURISM \\
\hline 48 XCA & $-0.0044 \%$ & $-1.1027 \%$ & $-3.3145 \%$ & $-0.1863 \%$ & $0.0000 \%$ & $-4.61 \%$ & HEAT \\
\hline 49 DOM & $-0.0006 \%$ & $-0.6860 \%$ & $-1.8276 \%$ & $-0.1301 \%$ & $-4.2142 \%$ & $-6.86 \%$ & TOURISM \\
\hline 50 JAM & $-0.0006 \%$ & $-0.3236 \%$ & $-2.3722 \%$ & $-0.1938 \%$ & $-8.4870 \%$ & $-11.38 \%$ & TOURISM \\
\hline 51 PRI & $-0.0006 \%$ & $-0.1014 \%$ & $-1.6726 \%$ & $-0.1793 \%$ & $-0.7814 \%$ & $-2.74 \%$ & HEAT \\
\hline 52 тто & $-0.0009 \%$ & $-0.1245 \%$ & $-2.4513 \%$ & $-0.1207 \%$ & $-2.1839 \%$ & $-4.88 \%$ & HEAT \\
\hline $53 \times C B$ & $-0.0017 \%$ & $-0.5995 \%$ & $-3.3617 \%$ & $-0.2107 \%$ & $-3.6624 \%$ & $-7.84 \%$ & TOURISM \\
\hline 54 AUT & $0.0000 \%$ & $0.0197 \%$ & $0.0000 \%$ & $-0.0472 \%$ & $1.9809 \%$ & $1.95 \%$ & TOURISM \\
\hline 55 BEL & $0.0000 \%$ & $0.0062 \%$ & $0.0000 \%$ & $-0.0482 \%$ & $1.2519 \%$ & $1.21 \%$ & TOURISM \\
\hline 56 CYP & $-0.0004 \%$ & $-0.4306 \%$ & $-0.1406 \%$ & $-0.0426 \%$ & $-3.9984 \%$ & $-4.61 \%$ & TOURISM \\
\hline 57 CZE & $0.0000 \%$ & $0.0369 \%$ & $0.0000 \%$ & $-0.0383 \%$ & $1.4414 \%$ & $1.44 \%$ & TOURISM \\
\hline 58 DNK & $0.0000 \%$ & $0.0271 \%$ & $0.0000 \%$ & $-0.0506 \%$ & $1.8480 \%$ & $1.82 \%$ & TOURISM \\
\hline 59 EST & $0.0000 \%$ & $0.1165 \%$ & $0.0000 \%$ & $-0.0379 \%$ & $2.1074 \%$ & $2.19 \%$ & TOURISM \\
\hline $60 \mathrm{FIN}$ & $0.0000 \%$ & $0.1317 \%$ & $0.0000 \%$ & $-0.0471 \%$ & $1.3954 \%$ & $1.48 \%$ & TOURISM \\
\hline 61 FRA & $0.0000 \%$ & $0.0002 \%$ & $0.0000 \%$ & $-0.0501 \%$ & $0.3515 \%$ & $0.30 \%$ & TOURISM \\
\hline 62 DEU & $0.0000 \%$ & $0.0115 \%$ & $0.0000 \%$ & $-0.0530 \%$ & $0.7933 \%$ & $0.75 \%$ & TOURISM \\
\hline 63 GRC & $-0.0001 \%$ & $-0.2039 \%$ & $-0.0545 \%$ & $-0.0329 \%$ & $-1.0597 \%$ & $-1.35 \%$ & TOURISM \\
\hline 64 HUN & $0.0000 \%$ & $0.0191 \%$ & $0.0000 \%$ & $-0.0376 \%$ & $0.9476 \%$ & $0.93 \%$ & TOURISM \\
\hline 65 IRL & $0.0000 \%$ & $0.0116 \%$ & $0.0000 \%$ & $-0.0404 \%$ & $0.7150 \%$ & $0.69 \%$ & TOURISM \\
\hline 66 ITA & $0.0000 \%$ & $-0.1355 \%$ & $0.0000 \%$ & $-0.0417 \%$ & $-0.0005 \%$ & $-0.18 \%$ & AGR \\
\hline 67 LVA & $0.0000 \%$ & $0.1817 \%$ & $0.0000 \%$ & $-0.0396 \%$ & $0.8261 \%$ & $0.97 \%$ & TOURISM \\
\hline 68 LTU & $0.0000 \%$ & $0.1642 \%$ & $0.0000 \%$ & $-0.0379 \%$ & $0.9750 \%$ & $1.10 \%$ & TOURISM \\
\hline 69 LUX & $0.0000 \%$ & $0.0057 \%$ & $0.0000 \%$ & $-0.0497 \%$ & $2.8828 \%$ & $2.84 \%$ & TOURISM \\
\hline $70 \mathrm{MLT}$ & $-0.0001 \%$ & $-0.1480 \%$ & $-0.0711 \%$ & $-0.0361 \%$ & $-6.2965 \%$ & $-6.55 \%$ & TOURISM \\
\hline
\end{tabular}


Table 7-2. Impact on GDP of $+3^{\circ} \mathrm{C}$ by country

\begin{tabular}{|c|c|c|c|c|c|c|c|c|}
\hline $\mathbf{N}$ & Code & SLR & AGR & HEAT & HEALTH & TOURISM & $\begin{array}{l}\text { Incidence on } \\
\text { GDP of }+3^{\circ} \mathrm{C}\end{array}$ & $\begin{array}{c}\text { Dominant } \\
\text { impact }\end{array}$ \\
\hline \multicolumn{2}{|c|}{\begin{tabular}{ll|} 
NLD \\
\end{tabular}} & $0.0000 \%$ & $0.0103 \%$ & $0.0000 \%$ & $-0.0506 \%$ & $0.7591 \%$ & $0.72 \%$ & TOURISM \\
\hline \multicolumn{2}{|c|}{72 POL } & $0.0000 \%$ & $0.0511 \%$ & $0.0000 \%$ & $-0.0405 \%$ & $0.9494 \%$ & $0.96 \%$ & TOURISM \\
\hline \multicolumn{2}{|c|}{73 PRT } & $0.0000 \%$ & $-0.1230 \%$ & $0.0000 \%$ & $-0.0486 \%$ & $-0.7612 \%$ & $-0.93 \%$ & TOURISM \\
\hline \multicolumn{2}{|c|}{74 SVK } & $0.0000 \%$ & $0.0359 \%$ & $0.0000 \%$ & $-0.0392 \%$ & $1.2305 \%$ & $1.23 \%$ & TOURISM \\
\hline \multicolumn{2}{|c|}{75 SVN } & $0.0000 \%$ & $0.0273 \%$ & $0.0000 \%$ & $-0.0523 \%$ & $1.3031 \%$ & $1.28 \%$ & TOURISM \\
\hline \multicolumn{2}{|c|}{76 ESP } & $0.0000 \%$ & $-0.1623 \%$ & $0.0000 \%$ & $-0.0521 \%$ & $-0.5523 \%$ & $-0.77 \%$ & TOURISM \\
\hline \multicolumn{2}{|c|}{77 SWE } & $0.0000 \%$ & $0.0566 \%$ & $0.0000 \%$ & $-0.0516 \%$ & $1.7159 \%$ & $1.72 \%$ & TOURISM \\
\hline \multicolumn{2}{|c|}{$78 \mathrm{GBR}$} & $0.0000 \%$ & $0.0099 \%$ & $0.0000 \%$ & $-0.0551 \%$ & $0.6373 \%$ & $0.59 \%$ & TOURISM \\
\hline \multicolumn{2}{|c|}{$79 \mathrm{CHE}$} & $0.0000 \%$ & $0.0151 \%$ & $0.0000 \%$ & $-0.0665 \%$ & $1.4678 \%$ & $1.42 \%$ & TOURISM \\
\hline & NOR & $-0.0001 \%$ & $0.0756 \%$ & $0.0000 \%$ & $-0.0487 \%$ & $1.4445 \%$ & $1.47 \%$ & TOURISM \\
\hline 81 & XEF & $0.0000 \%$ & $0.0364 \%$ & $0.0000 \%$ & $-0.0742 \%$ & $0.0000 \%$ & $-0.04 \%$ & HEALTH \\
\hline 82 & ALB & $-0.0002 \%$ & $-0.5880 \%$ & $-0.0018 \%$ & $-0.0837 \%$ & $-1.8545 \%$ & $-2.53 \%$ & TOURISM \\
\hline & $B G R$ & $0.0000 \%$ & $-0.2314 \%$ & $0.0000 \%$ & $-0.0836 \%$ & $1.0793 \%$ & $0.76 \%$ & TOURISM \\
\hline 84 & BLR & $0.0000 \%$ & $0.1365 \%$ & $0.0000 \%$ & $-0.1016 \%$ & $0.1481 \%$ & $0.18 \%$ & TOURISM \\
\hline 85 & HRV & $-0.0059 \%$ & $-0.1818 \%$ & $0.0000 \%$ & $-0.0475 \%$ & $-0.4174 \%$ & $-0.65 \%$ & TOURISM \\
\hline & ROU & $0.0000 \%$ & $0.0507 \%$ & $0.0000 \%$ & $-0.0406 \%$ & $0.2620 \%$ & $0.27 \%$ & TOURISM \\
\hline 87 & RUS & $-0.0001 \%$ & $0.2438 \%$ & $0.0000 \%$ & $-0.0620 \%$ & $1.2058 \%$ & $1.39 \%$ & TOURISM \\
\hline $8 \varepsilon$ & UKR & $0.0000 \%$ & $0.0614 \%$ & $0.0000 \%$ & $-0.0829 \%$ & $0.9421 \%$ & $0.92 \%$ & TOURISM \\
\hline 85 & XEE & $0.0000 \%$ & $0.0685 \%$ & $0.0000 \%$ & $-0.0887 \%$ & $0.0000 \%$ & $-0.02 \%$ & HEALTH \\
\hline 90 & XER & $0.0000 \%$ & $0.0479 \%$ & $0.0000 \%$ & $-0.0396 \%$ & $0.0000 \%$ & $0.01 \%$ & AGR \\
\hline 91 & KAZ & $0.0000 \%$ & $0.0489 \%$ & $0.0000 \%$ & $-0.0843 \%$ & $0.3404 \%$ & $0.31 \%$ & TOURISM \\
\hline 92 & KGZ & $0.0000 \%$ & $0.7822 \%$ & $0.0000 \%$ & $-0.0638 \%$ & $-1.7649 \%$ & $-1.05 \%$ & TOURISM \\
\hline 93 & XSU & $0.0000 \%$ & $0.1312 \%$ & $0.0000 \%$ & $-0.0568 \%$ & $0.0000 \%$ & $0.07 \%$ & AGR \\
\hline & ARM & $0.0000 \%$ & $0.2216 \%$ & $0.0000 \%$ & $-0.0714 \%$ & $0.0175 \%$ & $0.17 \%$ & AGR \\
\hline 95 & AZE & $0.0000 \%$ & $-0.5908 \%$ & $-0.0988 \%$ & $-0.0414 \%$ & $-0.1307 \%$ & $-0.86 \%$ & AGR \\
\hline & GEO & $-0.0003 \%$ & $0.1385 \%$ & $-0.0522 \%$ & $-0.0843 \%$ & $-1.9215 \%$ & $-1.92 \%$ & TOURISM \\
\hline & BHR & $-0.0005 \%$ & $-0.0683 \%$ & $-1.1748 \%$ & $-0.4204 \%$ & $-3.2314 \%$ & $-4.90 \%$ & TOURISM \\
\hline 98 & & $0.0000 \%$ & $-0.4277 \%$ & $-0.1860 \%$ & $-0.1181 \%$ & $-0.0843 \%$ & $-0.82 \%$ & AGR \\
\hline & ISR & $0.0000 \%$ & $-0.1655 \%$ & $-0.0400 \%$ & $-1.2584 \%$ & $-0.7563 \%$ & $-2.22 \%$ & HEALTH \\
\hline 100 & JOR & $0.0000 \%$ & $-0.3556 \%$ & $-0.1463 \%$ & $-0.5373 \%$ & $-4.0531 \%$ & $-5.09 \%$ & TOURISM \\
\hline 101 & KWT & $0.0000 \%$ & $-0.0182 \%$ & $-0.7005 \%$ & $-0.2407 \%$ & $-1.5365 \%$ & $-2.50 \%$ & TOURISM \\
\hline 102 & OMN & $0.0000 \%$ & $-0.0558 \%$ & $-0.7102 \%$ & $-0.3094 \%$ & $-1.5583 \%$ & $-2.63 \%$ & TOURISM \\
\hline 103 & QAT & $-0.0001 \%$ & $-0.0346 \%$ & $-1.2702 \%$ & $-0.3952 \%$ & $-0.8283 \%$ & $-2.53 \%$ & HEAT \\
\hline 104 & SAU & $0.0000 \%$ & $-0.0700 \%$ & $-1.4904 \%$ & $-0.5016 \%$ & $-1.2991 \%$ & $-3.36 \%$ & HEAT \\
\hline 105 & TUR & $-0.0001 \%$ & $-0.4687 \%$ & $0.0000 \%$ & $-0.3499 \%$ & $-0.0075 \%$ & $-0.83 \%$ & AGR \\
\hline 106 & ARE & $-0.0002 \%$ & $-0.1686 \%$ & $-1.3851 \%$ & $-0.4344 \%$ & $-2.8718 \%$ & $-4.86 \%$ & TOURISM \\
\hline 107 & XWs & $0.0000 \%$ & $-0.7620 \%$ & $-0.2868 \%$ & $-0.1673 \%$ & $0.0000 \%$ & $-1.22 \%$ & AGR \\
\hline 108 & EGY & $-0.0005 \%$ & $-1.1341 \%$ & $-0.6905 \%$ & $-0.4656 \%$ & $-1.5531 \%$ & $-3.84 \%$ & TOURISM \\
\hline 109 & MAR & $-0.0001 \%$ & $-1.1070 \%$ & $-0.0555 \%$ & $-0.7353 \%$ & $-1.8221 \%$ & $-3.72 \%$ & TOURISM \\
\hline 110 & TUN & $-0.0001 \%$ & $-0.7579 \%$ & $-0.2464 \%$ & $-0.5286 \%$ & $-1.5935 \%$ & $-3.13 \%$ & TOURISM \\
\hline 111 & XNF & $0.0000 \%$ & $-0.3463 \%$ & $-0.1242 \%$ & $-0.4551 \%$ & $0.0000 \%$ & $-0.93 \%$ & HEALTH \\
\hline 112 & BEN & $0.0000 \%$ & $-2.2061 \%$ & $-5.8667 \%$ & $-0.7895 \%$ & $-4.7655 \%$ & $-13.63 \%$ & HEAT \\
\hline 113 & BFA & $0.0000 \%$ & $-2.3843 \%$ & $-5.8824 \%$ & $-0.6710 \%$ & $-2.9330 \%$ & $-11.87 \%$ & HEAT \\
\hline 114 & CMR & $0.0000 \%$ & $-2.4157 \%$ & $-2.6122 \%$ & $-1.0351 \%$ & $-2.0672 \%$ & $-8.13 \%$ & HEAT \\
\hline 115 & $\mathrm{CIV}$ & $0.0000 \%$ & $-2.6715 \%$ & $-7.3540 \%$ & $-1.1743 \%$ & $-1.8351 \%$ & $-13.03 \%$ & HEAT \\
\hline 116 & $\mathrm{GHA}$ & $0.0000 \%$ & $-2.5318 \%$ & $-7.6143 \%$ & $-1.2015 \%$ & $-1.8443 \%$ & $-13.19 \%$ & HEAT \\
\hline 117 & GIN & $0.0000 \%$ & $-2.2525 \%$ & $-2.4491 \%$ & $-0.5128 \%$ & $-4.9416 \%$ & $-10.16 \%$ & TOURISM \\
\hline 118 & NGA & $0.0000 \%$ & $-4.0968 \%$ & $-8.2096 \%$ & $-0.9791 \%$ & $-0.6444 \%$ & $-13.93 \%$ & HEAT \\
\hline 119 & SEN & $0.0000 \%$ & $-1.5615 \%$ & $-3.6766 \%$ & $-0.6634 \%$ & $-3.6789 \%$ & $-9.58 \%$ & TOURISM \\
\hline 120 & TGO & $0.0000 \%$ & $-2.9926 \%$ & $-6.7908 \%$ & $-0.8777 \%$ & $-7.6318 \%$ & $-18.29 \%$ & TOURISM \\
\hline 121 & $X W F$ & $0.0000 \%$ & $-1.5088 \%$ & $-3.9966 \%$ & $-0.5685 \%$ & $0.0000 \%$ & $-6.07 \%$ & HEAT \\
\hline 122 & XCF & $0.0000 \%$ & $-0.4709 \%$ & $-0.8100 \%$ & $-0.6294 \%$ & $-0.4675 \%$ & $-2.38 \%$ & HEAT \\
\hline 123 & XAC & $0.0000 \%$ & $-0.9863 \%$ & $-0.0461 \%$ & $-0.7702 \%$ & $0.0000 \%$ & $-1.80 \%$ & AGR \\
\hline 124 & ETH & $0.0000 \%$ & $-3.4512 \%$ & $0.0000 \%$ & $-0.8943 \%$ & $-1.4763 \%$ & $-5.82 \%$ & AGR \\
\hline 125 & KEN & $0.0000 \%$ & $-2.8648 \%$ & $-0.1698 \%$ & $-0.9299 \%$ & $-1.6563 \%$ & $-5.62 \%$ & AGR \\
\hline 126 & MDG & $-0.0002 \%$ & $-2.9062 \%$ & $-2.5131 \%$ & $-0.9861 \%$ & $-3.5947 \%$ & $-10.00 \%$ & TOURISM \\
\hline 127 & MWI & $0.0000 \%$ & $-2.6408 \%$ & $-1.5485 \%$ & $-0.9422 \%$ & $-4.6332 \%$ & $-9.76 \%$ & TOURISM \\
\hline 128 & MUS & $-0.0009 \%$ & $-0.7158 \%$ & $-2.1495 \%$ & $-0.8996 \%$ & $-8.3783 \%$ & $-12.14 \%$ & TOURISM \\
\hline 129 & $\mathrm{MOZ}$ & $-0.0001 \%$ & $-1.1773 \%$ & $-2.7128 \%$ & $-0.9728 \%$ & $-3.0178 \%$ & $-7.88 \%$ & TOURISM \\
\hline 130 & RWA & $0.0000 \%$ & $-3.7427 \%$ & $0.0000 \%$ & $-1.0299 \%$ & $-4.8945 \%$ & $-9.67 \%$ & TOURISM \\
\hline 131 & TZA & $-0.0001 \%$ & $-2.5945 \%$ & $-1.4315 \%$ & $-1.0207 \%$ & $-3.7480 \%$ & $-8.79 \%$ & TOURISM \\
\hline 132 & UGA & $0.0000 \%$ & $-2.3230 \%$ & $-0.3320 \%$ & $-0.8564 \%$ & $-4.0730 \%$ & $-7.58 \%$ & TOURISM \\
\hline 133 & ZMB & $0.0000 \%$ & $-1.1479 \%$ & $-0.4776 \%$ & $-1.1182 \%$ & $-1.5571 \%$ & $-4.30 \%$ & TOURISM \\
\hline 134 & ZWE & $0.0000 \%$ & $-0.9073 \%$ & $-0.1290 \%$ & $-0.7963 \%$ & $-2.9613 \%$ & $-4.79 \%$ & TOURISM \\
\hline 135 & XEC & $0.0000 \%$ & $-1.2136 \%$ & $-2.4070 \%$ & $-0.9955 \%$ & $0.0000 \%$ & $-4.62 \%$ & HEAT \\
\hline 136 & BWA & $0.0000 \%$ & $-0.5257 \%$ & $-0.3916 \%$ & $-0.7531 \%$ & $-1.7062 \%$ & $-3.38 \%$ & TOURISM \\
\hline 137 & NAM & $0.0000 \%$ & $-0.9395 \%$ & $-0.0747 \%$ & $-0.7110 \%$ & $-3.3564 \%$ & $-5.08 \%$ & TOURISM \\
\hline 138 & ZAF & $0.0000 \%$ & $-0.2159 \%$ & $-0.0003 \%$ & $-0.8577 \%$ & $-0.5198 \%$ & $-1.59 \%$ & HEALTH \\
\hline 139 & XSC & $0.0000 \%$ & $-1.0459 \%$ & $-0.0015 \%$ & $-0.7656 \%$ & $0.0000 \%$ & $-1.81 \%$ & AGR \\
\hline 140 & XTW & $-0.0013 \%$ & $-0.1252 \%$ & $0.0000 \%$ & $-0.7543 \%$ & $0.0000 \%$ & $-0.88 \%$ & HEALTH \\
\hline
\end{tabular}


It is also evident that effects are similar among similar countries, that is when they belong to the same region or are characterized by comparable socio-economic conditions. Figure 7 presents a scatter plot of total percentage variations of GDP against per capita income levels. The correlation between these two variables is positive and as large as 0.445 , confirming a robust finding from previous studies (e.g. Eboli, Parrado and Roson, 2010, Roson and van der Mensbrugghe, 2012) that climate change impacts act like a highly regressive tax, making poor countries poorer, and sometimes rich countries richer.

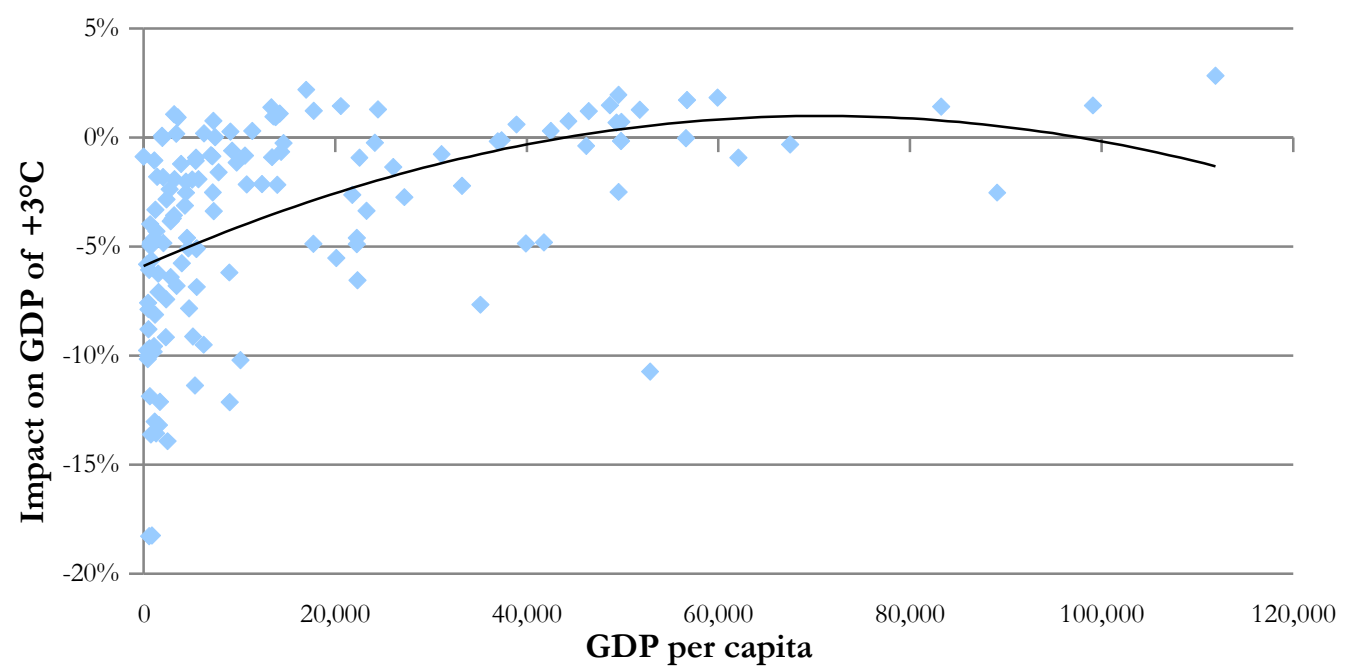

Figure 7. Percentage variation of GDP against per capita income level

It is known that economic development is itself correlated with geographical location and temperature: in contemporary data, national income falls $8.5 \%$ per degree Celsius in the world cross-section (Dell, Jones and Olken, 2009). We do not discuss here any causality or interpretation for this correlation. Rather, we show in Figures 8 and 9 another two scatter plots, this time contrasting GDP variations with average temperature and latitude. The corresponding correlation factors are, respectively, -0.785 and 0.732 .

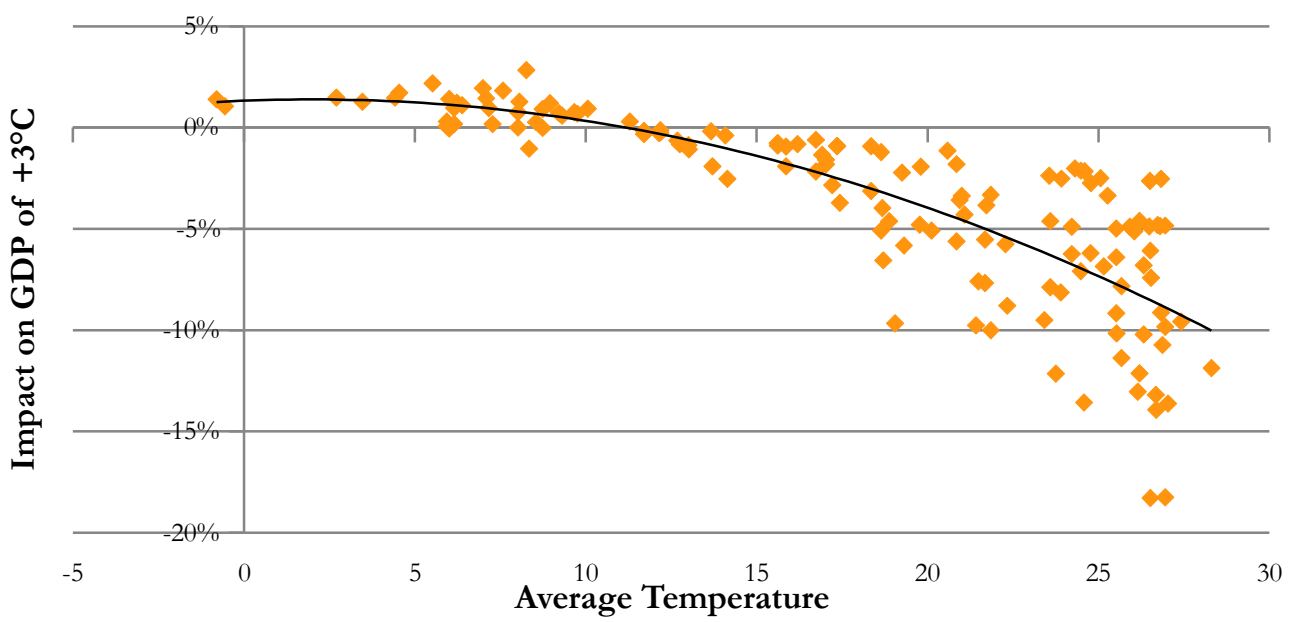

Figure 8. Percentage variation of GDP against average temperature 


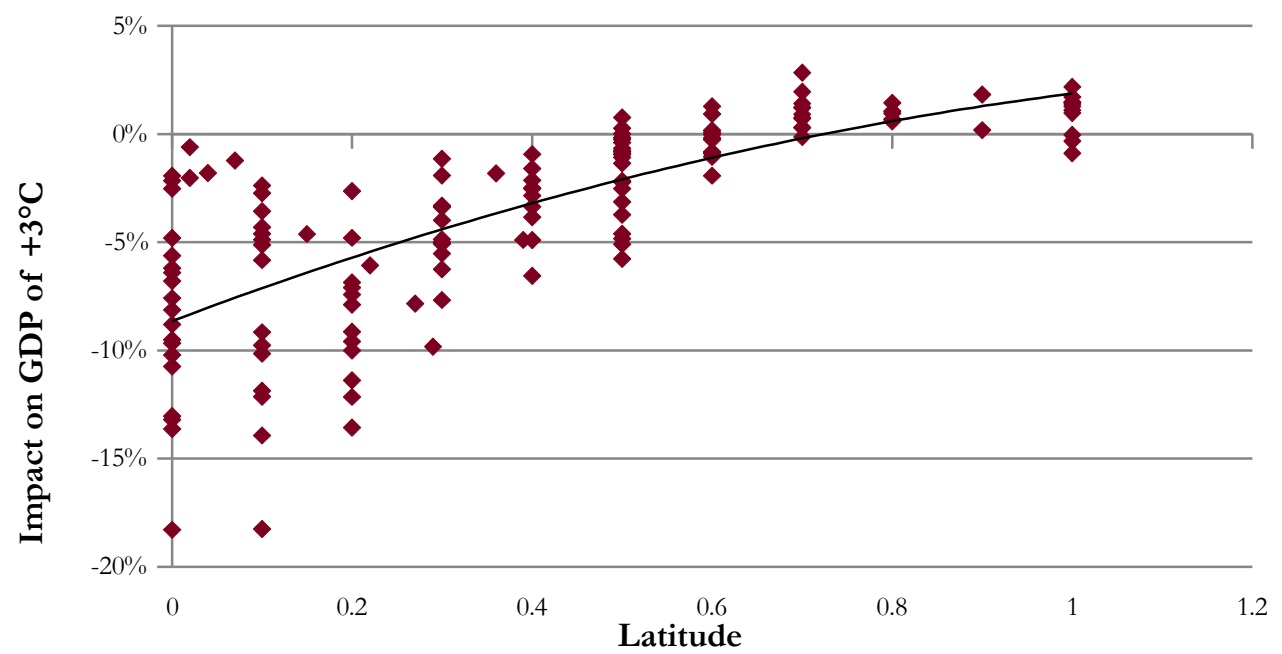

Figure 9. Percentage variation of GDP against latitude

\section{Conclusion}

In this paper, a new set of climate change damage functions has been presented, improving earlier estimates in several ways. First, functions and parameters are provided with a large regional disaggregation (140 countries) and in a format which, by referring to the latest GTAP social accounting matrix, makes them easily employable in many CGE and CGE-based models. Information from new, recently available studies, typically from the non economic literature, has been processed in such a way that parameter values for economic variables, like labor productivity, can be estimated. Because of the wealth of primary data utilized in this exercise, it has also been possible to detect non-linearities in many impacts of climate change.

Although our estimates are mostly intended for use in multi-sectoral macroeconomic models, we undertook a simple aggregation procedure to verify the order of magnitude of the various impacts, as well as their distribution. Our findings confirm that the negative effects of climate change will be mainly borne by developing countries, located in tropical regions. 


\section{References}

AgMIP (2014). Guide for Regional Integrated Assessments: Handbook of Methods and Procedures Version 5.1.

Åström, C., Rocklöv, J., Hales, S., Béguin, A., Louis V., and Sauerborn, R. (2012). "Potential Distribution of Dengue Fever Under Scenarios of Climate Change and Economic Development", EcoHealth, 9: 448-454.

Béguin, A., Hales, S., Rocklöv, J., Åström, C., Louis, V., and Sauerborn, R. (2011). "The opposing effects of climate change and socio-economic development on the global distribution of malaria", Global Environmental Change, 21: 1209-1214.

Berrittella, M., Bigano, A., Roson, R. and Tol, R.S.J (2006). "A General Equilibrium Analysis of Climate Change Impacts on Tourism", Tourism Management, 25(5): 913-924.

Bigano, A., Bosello, F., Roson, R. and Tol, R.S.J. (2008). "Economy-wide Impacts of Climate Change: a Joint Analysis for Sea Level Rise and Tourism", Mitigation and Adaptation Strategies for Climate Change, 13(8): 765-791.

Bosello, F., Roson, R., and Tol, R.S.J. (2006). "Economy-wide estimates of the implications of climate change: Human health", Ecological Economics, vol. 58, pp. 579-591.

Cline, W.R. (2007). Global Warming and Agriculture - Impact Estimates by Country, Center for Global Development, Peterson Institute for International Economics, Washington D.C.

De Cian, E., Lanzi, E. and Roson, R. (2013). "Seasonal temperature variations and energy demand A panel cointegration analysis for climate change impact assessment", Climatic Change, 116: 805-825.

Dell, M., B.F. Jones and B.A. Olken (2009) "Temperature and Income: Reconciling New CrossSectional and Panel Estimates," American Economic Review Papers and Proceedings, 99:198-204.

Deke, O., Hooss, K.G., Kasten, C., Klepper, G. and Springer, K. (2001), Economic impact of climate change: simulations with a regionalized climate-economy model, Kiel Working Papers 1065, Kiel Institute for the World Economy.

Dell, M., Jones, B.F. and Olken, B.A. (2014) "What Do We Learn from the Weather? The New Climate-Economy Literature", Journal of Economic Literature, 52(3): 740-798.

Ebi, K.L., and Mills, D. (2013). "Winter mortality in a warming climate", WIREs Climate Change, doi: 10.1002/wcc.211.

Eboli, F., Parrado, R. and Roson, R. (2010), "Climate change feedback on economic growth: explorations with a dynamic general equilibrium model", Environment and Development Economics, 15(5):515-533.

Hamilton, J.M., Maddison, D.J., and Tol, R.SJ. (2005). "Climate change and international tourism: A simulation study”, Global Environmental Change, 15: 253-266.

Honda, Y., Kondo, M., McGregor, G., Kim, H., Guo, Y., Hijioka, Y., Yoshikawa, M., Oka, K., Takano, S., Hales, S., and Kovats, R.S. (2013). "Heat-related mortality risk model for climate change impact projection", Environmental Health and Preventive Medicine, doi:10.1007/s12199-013-0354-6.

Hyatt, O.M., Lemke, B. and Kjellström, T. (2010). "Regional maps of occupational heat exposure: past, present, and potential future", Global Health Action, 3: 5715 - DOI: 10.3402/gha.v3i0.5715.

International Monetary Fund (2014). Balance of Payment Statistics Yearbook 2014, IMF.

IPCC (2014), Climate Change 2014: Impacts, Adaptation, and Vulnerability. Part A: Global and Sectoral Aspects. Contribution of Working Group II to the Fifth Assessment Report of the Inter-governmental Panel on Climate Change, Cambridge University Press.

Kjellström, T., Kovats, R.S., Lloyd, S.J., Holt, T., and Tol, R.S.J. (2009). "The direct impact of climate change on regional labor productivity", Archives of Environ. Occup. Health, 64: 217-27. 
Kolstad, E., and Johansson, K.A. (2011). "Uncertainties associated with quantifying climate change impacts on human health: a case study for diarrhea", Environmental Health Perspectives, 119(3): 299-305.

Lemke, B., and Kjellström, T. (2012). "Calculating Workplace WBGT from Meteorological Data: A Tool for Climate Change Assessment", Industrial Health, vol.50, pp.267-278.

Manne, A., Mendelsohn, R. and R.G. Richels (1995), "MERGE: A Model for Evaluating Regional and Global Effects of GHG Reduction Policies.", Energy Policy, 23(1):17-34.

Martens, W.J.M. (1998). “Climate Change, Thermal Stress and Mortality Changes”, Social Science and Medicine, 46(3): 331-344.

Martens, W.J.M., Jetten, T.H., and Focks, D.A. (1997). "Sensitivity of Malaria, Schistomiasis and Dengue to Global Warming", Climatic Change, 35: 145-156.

Mendelsohn R., and Schlesinger M.E. (1999), "Climate Response Functions”, Ambio, 28(4): 362-366.

Nordhaus, W.D. (1994), "Expert opinion on climate change”, American Science, 82(1):45-51.

Nordhaus, W.D. and J. Boyer (1999), Roll the Dice again: Economic Models of Global Warming, MIT Press.

Nordhaus, W.D. and Z. Yang (1996), "A Regional Dynamic General-Equilibrium Model of Alternative Climate Change Strategies”, American Economic Review, 86(4):741-765.

Pant, H. (2002), Global Trade and Environment Model (GTEM): A computable general equilibrium model of the global economy and environment, mimeo, Australian Bureau of Agricultural and Resource Economics, Canberra.

Peck, S. and T. Teisberg (1992), “CETA a model for carbon emission trajectory assessment”, Energy Journal, 13(1): 55-78.

Roson, R., and Sartori, M. (2010). The ENVironmental Impact and Sustainability Applied General Equilibrium (ENVISAGE) Model - Introducing Climate Change Impacts and Adaptation, The World Bank, Washington D.C.

Roson, R., and van der Mensbrugghe, D. (2012). "Climate Change and Economic Growth: Impacts and Interactions”, International Journal of Sustainable Economy, 4(3): 270-285

Tawasupa, B., Yiengprugsawan, V., Kjellström, T., Berecki-Gisolf, J., Seubsman S., and Sleigh, A. (2013). "Association between Heat Stress and Occupational Injury among Thai Workers: Findings of the Thai Cohort Study", Industrial Health, 51: 34-46.

Tol, R.S.J. (2002), "Estimates of the Damage Costs of Climate Change - Part 1: Benchmark Estimates", Environmental and Resource Economics, 21: 47-73.

Tol, R.S.J. (2015), "Who Benefits and Who Loses from Climate Change?”, Handbook of Climate Change Mitigation and Adaptation, Springer Science Business Media, New York. Forthcoming.

Weitzman, M. (2010), "What is the "Damages Function" for Global Warming - and What Difference Might It Make?", Climate Change Economics, 1(1): 57-69.

WHO (2013), Impact of Dengue, World Health Organization (WHO), Geneva, Switzerland, www.who.int/csr/disease/dengue/impact/en/.

Zhou, X., Yang, G., Yang, K., Wang, X., Hong, Q., Sun, L., Malone, J.B., Kristensen, T.K., Bergquist, N.R., and Utzinger, J. (2008). "Potential impact of climate change on schistosomiasis transmission in China", American Journal of Tropical Medicine and Hygiene, 78(2): 188-194. 


\section{Appendix}

Table A0. Country codes.

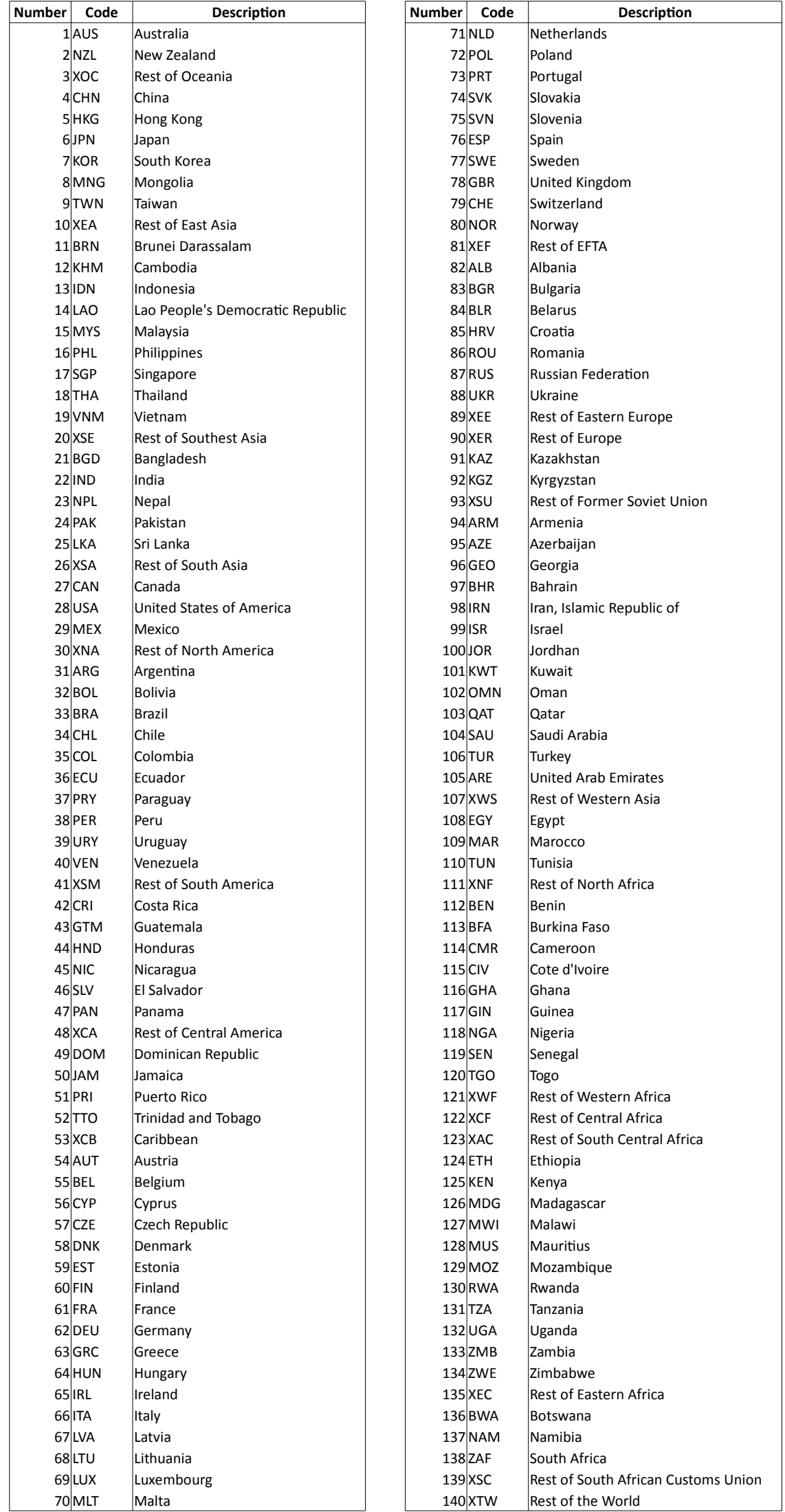


Table A1. Sea level rise: percentage change of land stock by meter of SLR and VLM. Countries with asterisk do not have coastline.

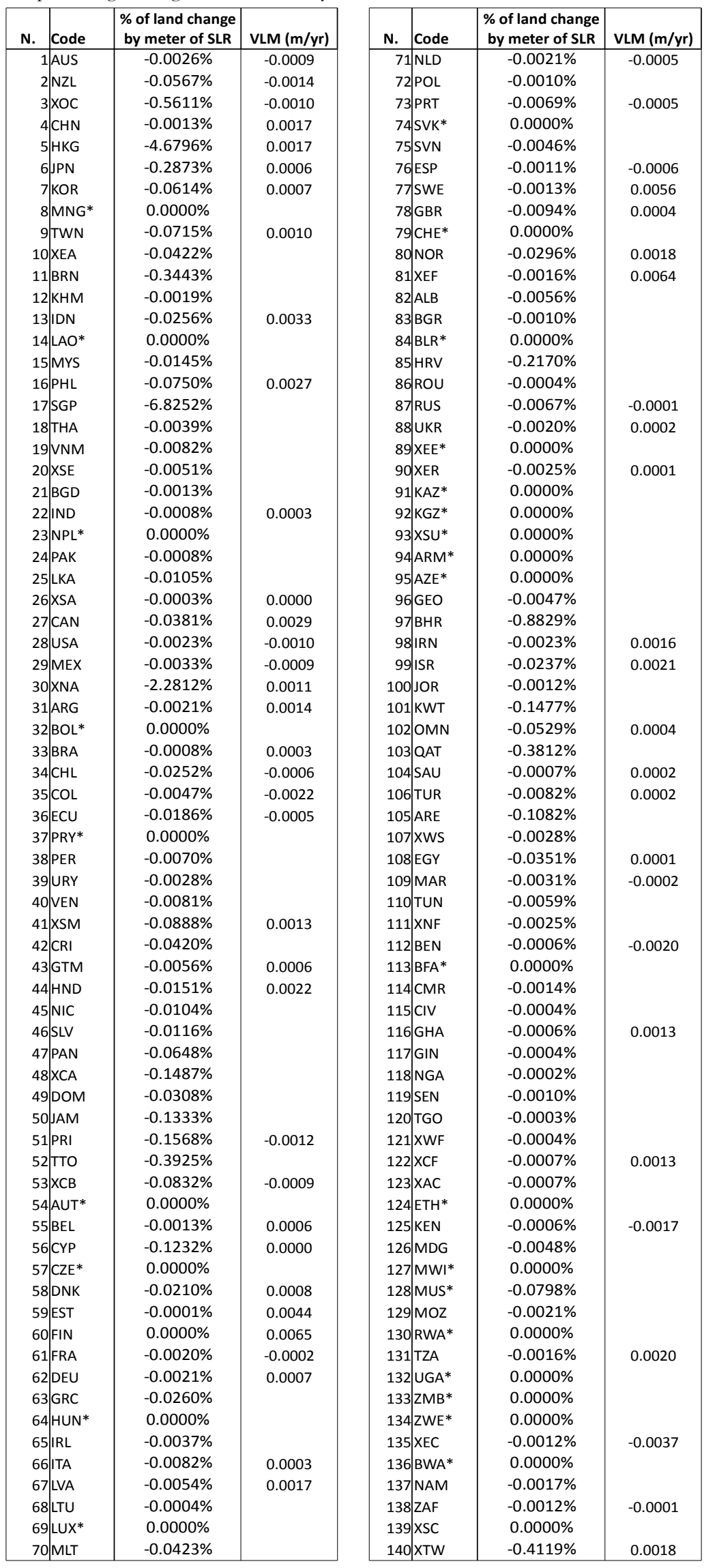


Table A2-1. Sea level rise: percentage losses of land for $+1,+2,+3,+4$ and $+5{ }^{\circ} \mathrm{C}$, years 2050 and 2100 . Values lower than $-0.1 \%$ in red.

\begin{tabular}{|c|c|c|c|c|c|c|c|c|c|c|}
\hline & \multicolumn{5}{|c|}{50} & \multicolumn{5}{|c|}{2100} \\
\hline Code & $+1^{\circ} \mathrm{C}$ & $+2^{\circ} \mathrm{C}$ & $+3^{\circ} \mathrm{C}$ & $+4^{\circ} \mathrm{C}$ & $55^{\circ} \mathrm{C}$ & $+1^{\circ} \mathrm{C}$ & $+2^{\circ} \mathrm{C}$ & $+3^{\circ} \mathrm{C}$ & $+4^{\circ} \mathrm{C}$ & - \\
\hline 1 AUS & $0.0007 \%$ & $-0.0011 \%$ & $-0.0016 \%$ & $.0020 \%$ & $-0.0025 \%$ & $-0.0014 \%$ & $-0.0023 \%$ & $0.0032 \%$ & $-0.0041 \%$ & 0.0050 \\
\hline 2 NZL & $-0.0163 \%$ & $-0.0260 \%$ & $-0.0357 \%$ & $-0.0454 \%$ & $-0.0551 \%$ & $-0.0326 \%$ & $-0.0520 \%$ & $-0.0714 \%$ & $-0.0908 \%$ & 0110 \\
\hline $3 \times 0 C$ & $505 \%$ & $-0.2465 \%$ & $.3424 \%$ & 438 & .534 & $-0.3009 \%$ & $-0.4929 \%$ & -0.68 & $-0.8769 \%$ & $1.0688 \%$ \\
\hline $4 \mathrm{CHN}$ & $-0.0002 \%$ & $-0.0004 \%$ & $-0.0006 \%$ & $-0.0008 \%$ & $-0.0010 \%$ & $-0.0003 \%$ & $-0.0008 \%$ & $-0.0012 \%$ & $-0.0016 \%$ & $-0.0021 \%$ \\
\hline 5 HKG & $-0.6151 \%$ & $-1.4156 \%$ & $-2.2161 \%$ & $-3.0166 \%$ & $-3.8171 \%$ & $-1.2302 \%$ & $-2.8312 \%$ & $-4.4322 \%$ & $-6.0333 \%$ & $-7.6343 \%$ \\
\hline 6 JPN & $-0.0549 \%$ & $-0.1041 \%$ & $-0.1532 \%$ & $-0.2023 \%$ & $-0.2515 \%$ & $-0.1098 \%$ & $-0.2081 \%$ & $-0.3064 \%$ & $-0.4047 \%$ & $-0.5030 \%$ \\
\hline 7) KOR & $-0.0112 \%$ & $-0.0217 \%$ & $-0.0322 \%$ & $-0.0427 \%$ & $-0.0532 \%$ & $-0.0224 \%$ & $-0.0433 \%$ & $-0.0643 \%$ & $-0.0853 \%$ & $-0.1063 \%$ \\
\hline 8 MNG* & $0.0000 \%$ & $0.0000 \%$ & $0.0000 \%$ & $0.0000 \%$ & $0.0000 \%$ & $0.0000 \%$ & $0.0000 \%$ & $0.0000 \%$ & $0.0000 \%$ & $0.0000 \%$ \\
\hline 9 TWN & $-0.0121 \%$ & $-0.0243 \%$ & $-0.0366 \%$ & $-0.0488 \%$ & $-0.0610 \%$ & $-0.0242 \%$ & $-0.0487 \%$ & $-0.0731 \%$ & $-0.0976 \%$ & $1221 \%$ \\
\hline 10XEA & $-0.0092 \%$ & $-0.0164 \%$ & $-0.0237 \%$ & $-0.0309 \%$ & $-0.0381 \%$ & $-0.0185 \%$ & $-0.0329 \%$ & $-0.0473 \%$ & $-0.0617 \%$ & $-0.0762 \%$ \\
\hline 11 BRN & $-0.0753 \%$ & $-0.1342 \%$ & $-0.1931 \%$ & $-0.2520 \%$ & $-0.3109 \%$ & $-0.1506 \%$ & $-0.2684 \%$ & $-0.3862 \%$ & $-0.5040 \%$ & $-0.6217 \%$ \\
\hline 12 KHM & $-0.0004 \%$ & $-0.0008 \%$ & $-0.0011 \%$ & $-0.0014 \%$ & $-0.0018 \%$ & $-0.0009 \%$ & $-0.0015 \%$ & $-0.0022 \%$ & $-0.0028 \%$ & $-0.0035 \%$ \\
\hline 13 |IDN & -0.00 & $-0.0058 \%$ & & -0.01 & -0.018 & & & & & \\
\hline 14 LAO* & $0.0000 \%$ & $0.0000 \%$ & $0.0000 \%$ & $0.0000 \%$ & $0.0000 \%$ & $0.0000 \%$ & $0.0000 \%$ & $0.0000 \%$ & $0.0000 \%$ & $0.0000 \%$ \\
\hline 15 MYS & $-0.0032 \%$ & $-0.0056 \%$ & $-0.0081 \%$ & $-0.0106 \%$ & $-0.0131 \%$ & $-0.0063 \%$ & $-0.0113 \%$ & $-0.0162 \%$ & $-0.0212 \%$ & $-0.0262 \%$ \\
\hline 16 PHL & -0.00 & $-0.0192 \%$ & $-0.0320 \%$ & $-0.0449 \%$ & $-0.0577 \%$ & $-0.0128 \%$ & $-0.0384 \%$ & & $-0.0897 \%$ & -0.115 \\
\hline 17 SGP & $-1.4932 \%$ & $-2.6608 \%$ & $-3.8283 \%$ & $-4.9959 \%$ & $-6.1634 \%$ & & & & $-9.9917 \%$ & $12.3268 \%$ \\
\hline THA & -0.00 & $-0.0015 \%$ & -0.00 & $-0.0029 \%$ & $-0.0035 \%$ & $-0.0017 \%$ & -0.0 & $-0.0044 \%$ & $-0.0057 \%$ & $-0.0071 \%$ \\
\hline 19 VNM & -0.00 & -0.00 & $-0.0046 \%$ & $-0.0060 \%$ & $0.0074 \%$ & -0.0 & & -0.0 & $-0.0120 \%$ & $.0148 \%$ \\
\hline 20|XSE & -0.00 & & -0.00 & $-0.0037 \%$ & $-0.0046 \%$ & & & & & $0.0092 \%$ \\
\hline $21 \mid B G D$ & -0.0 & & -0 . & $-0 . c$ & -0.0 & & & -0.0 & -0.00 & $-0.0023 \%$ \\
\hline $22 \mid$ IND & $-0.0002 \%$ & $-0.0003 \%$ & $-0.0004 \%$ & $-0.0006 \%$ & $-0.0007 \%$ & $-0.0003 \%$ & $-0.0006 \%$ & $-0.0009 \%$ & $-0.0011 \%$ & $-0.0014 \%$ \\
\hline 23 NPL* & $0.0000 \%$ & $0.0000 \%$ & $0.0000 \%$ & $0.0000 \%$ & $0.0000 \%$ & $0.0000 \%$ & $00 \%$ & 0.0 & $0.0000 \%$ & $0000 \%$ \\
\hline 24|PAK & $-0.0002 \%$ & -0.0 & $-0.0005 \%$ & $-0.0006 \%$ & $-0.0007 \%$ & $4 \%$ & & & $\%$ & $5 \%$ \\
\hline 25 LKA & -0.0 & -0.0 & -0.00 & $-0.0077 \%$ & $-0.0095 \%$ & -0.00 & & & $-0.0154 \%$ & $-0.0189 \%$ \\
\hline 26 XSA & 0.0001\% & $-0.0001 \%$ & $-0.0002 \%$ & $-0.0002 \%$ & $-0.0003 \%$ & $-0.0001 \%$ & $-0.0003 \%$ & -0.0 & $-0.0005 \%$ & $-0.0006 \%$ \\
\hline 27 CAN & -0.00 & & -0.01 & $-0.0223 \%$ & $-0.0288 \%$ & & & & $-0.0445 \%$ & $0.0576 \%$ \\
\hline 28 USA & -0.0 & & & -0.0 & $-0.0022 \%$ & & & & $-0.0037 \%$ & $-0.0045 \%$ \\
\hline MEX & .00 & -0.0 & -0.00 & $-0.0026 \%$ & $0.0031 \%$ & -0.00 & -0.6 & -0.0 & -0.00 & -0.0063 \\
\hline 30|XNA & & & & & & & & & -3.0 & $3 \%$ \\
\hline ARG & -0.00 & -0.0 & -0.0 & -0.00 & $-0.0018 \%$ & & & & -0.00 & -0.0036 \\
\hline 32 BOL* & $0.0000 \%$ & $0.0000 \%$ & $0.0000 \%$ & $0.0000 \%$ & $0.0000 \%$ & $0 \%$ & $0 \%$ & $0 . c$ & $0.0000 \%$ & $.0000 \%$ \\
\hline 33 BRA & $-0.0002 \%$ & $-0.0003 \%$ & $-0.0004 \%$ & $-0.0006 \%$ & 0.000 & -0.0 & -0. & -0.0 & -0.001 & $0014 \%$ \\
\hline $34 \mathrm{CHL}$ & -0.006 & -0.0 & $\%$ & & -0.0 & & & & 0 & $-0.0470 \%$ \\
\hline $35 \mid \mathrm{COL}$ & -0.0 & & -0.00 & -0.00 & -0.00 & & & & -0.00 & -0.0 \\
\hline 36 ECU & -0.00 & -0.0 & -0.01 & $-0.0141 \%$ & -0.017 & -0.00 & -0.0 & -0.0 & -0.02 & -0.03 \\
\hline 37 PRY* & & & & & & & & & & \\
\hline$R$ & & & & & & & & & & \\
\hline 39 URY & -0.00 & -0.00 & -0.00 & $-0.0020 \%$ & -0.002 & -0.00 & $-0.0022 \%$ & -0.0 & -0.00 & $-0.0050^{\circ}$ \\
\hline 40|VEN & -0.00 & $-0.0032 \%$ & $-0.0045 \%$ & $-0.0059 \%$ & -0.007 & -0.0 & -0. & -0.0 & -0.01 & $-0.0146 \%$ \\
\hline 41 XSM & -0.0 & & & -0.0 & & & & & & \\
\hline CRI & -0.0 & -0.0 & -0.0 & -0.03 & 0.03 & & & & -0.0 & -0.0758 \\
\hline 43 GTM & $-0.0011 \%$ & -0.0 & $-0 . c$ & -0.0 & 0.6 & -0. & -0 & -0.0 & -0.00 & $0.0097 \%$ \\
\hline 44|HND & -0.0 & & & -0.0 & & & & & -0.0 & $-0.0240 \%$ \\
\hline c & & -0.0 & & & -0.0 & & & & & 0.0187 \\
\hline 46) SLV & $-0.0025 \%$ & -0.0 & -0.00 & -0.00 & -0.010 & -0.0 & -0. & -0.0 & -0.01 & -0.0209 \\
\hline 47 PAN & & & & & & & & & -0 . & $-0.1170 \%$ \\
\hline & & & & & & & & & & \\
\hline DOM & $-0 . c$ & -0. & -0. & -0.02 & -0.0 & -0. & $-0 . c$ & & -0.0 & -0. \\
\hline JAM & & & & -0.0 & -0.1 & & & & & $-0.2407 \%$ \\
\hline & & & & & & & & & & $16 \%$ \\
\hline TTT & & & & & & & & & & 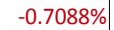 \\
\hline 53 XCB & -0.0 & -0.0 & -0.0 & & -0. & $\%$ & -0.0 & -0.1 & -0.1 & $1580 \%$ \\
\hline AUT* & & $\%$ & & & $\%$ & & & 0.00 & $0 \%$ & $000 \%$ \\
\hline & & & & & & & & & & \\
\hline & & & & & & & & & & \\
\hline 57 CZE* & & & $0.0000 \%$ & $0 \%$ & 0.0 & $0 \%$ & $0 \%$ & $0.0000 \%$ & $0000 \%$ & $0000 \%$ \\
\hline & & -0.007 & & & & & & & & $3 \%$ | \\
\hline & & & & & & & & & & \\
\hline & & & & & & & & & $00 \%$ & $000 \%$ \\
\hline RA & & -0.0 & -0.0 & & & & & & & $36 \%$ \\
\hline & & & & & & & & & & \\
\hline & & -0.0 & & & & & & & & \\
\hline flnovis & & $0.0000 \%$ & $0.0000 \%$ & $0.0000 \%$ & $0.0000 \%$ & $0.0000 \%$ & $0.0000 \%$ & 0.000 & 00 & $0000 \%$ \\
\hline & & -0.0 & & & & $\%$ & & & & \\
\hline & & & & & & & & & & \\
\hline 67|LVA & $-0.0007 \%$ & $-0.0017 \%$ & $-0.0026 \%$ & $-0.0035 \%$ & -0.00 & $-0.0015 \%$ & $-0.0033 \%$ & -0.00 & -0.00 & $-0.0089 \%$ \\
\hline & -0.0 & -0.0002 & -0.0002 & & -0.00 & $-0.0002 \%$ & -0.00 & -0.0 & -0.0 & \\
\hline & & & & & & & & & & \\
\hline & 0.00 & -0.0 & -0.0 & -0.030 & -0.038 & $-0.0185 \%$ & $-0.0350 \%$ & $-0.04 / 4 \%$ & $-0.0619 \%$ & \\
\hline
\end{tabular}


Table A2-2. Sea level rise: percentage losses of land for $+1,+2,+3,+4$ and $+5{ }^{\circ} \mathrm{C}$, years 2050 and 2100 . Values lower than $-0.1 \%$ in red.

\begin{tabular}{|c|c|c|c|c|c|c|c|c|c|c|}
\hline & \multicolumn{5}{|c|}{2050} & \multicolumn{5}{|c|}{2100} \\
\hline Code & $+1^{\circ} \mathrm{C}$ & $+2^{\circ} \mathrm{C}$ & $+3^{\circ} \mathrm{C}$ & $+4^{\circ} \mathrm{C}$ & $+5^{\circ} \mathrm{C}$ & $+1^{\circ} \mathrm{C}$ & $+2^{\circ} \mathrm{C}$ & $+3^{\circ} \mathrm{C}$ & $+4^{\circ} \mathrm{C}$ & TS \\
\hline 71 NLD & $-0.0005 \%$ & $-0.0009 \%$ & $-0.0012 \%$ & $-0.0016 \%$ & $-0.0019 \%$ & $-0.0010 \%$ & $-0.0017 \%$ & $-0.0024 \%$ & $-0.0031 \%$ & -0.00389 \\
\hline $\mathrm{PO}$ & $-0.0002 \%$ & $-0.0004 \%$ & $-0.0006 \%$ & $-0.0007 \%$ & $-0.0009 \%$ & $-0.0004 \%$ & $-0.0008 \%$ & $-0.0011 \%$ & $-0.0014 \%$ & $-0.0018 \%$ \\
\hline 3 PRT & $-0.0017 \%$ & $-0.0029 \%$ & $-0.0041 \%$ & $-0.0053 \%$ & $-0.0064 \%$ & $-0.0034 \%$ & $-0.0058 \%$ & $-0.0081 \%$ & -0.01 & $-0.0129 \%$ \\
\hline $74 \mid \mathrm{SVK}^{*}$ & $0.0000 \%$ & $0.0000 \%$ & $0.0000 \%$ & $0.0000 \%$ & $0.0000 \%$ & $0.0000 \%$ & $0.0000 \%$ & $0.0000 \%$ & $0.0000 \%$ & $0.0000 \%$ \\
\hline 75 SVN & $-0.0010 \%$ & $-0.0018 \%$ & $-0.0026 \%$ & $-0.0034 \%$ & $-0.0041 \%$ & $-0.0020 \%$ & $-0.0036 \%$ & $-0.0051 \%$ & $-0.0067 \%$ & $-0.0083 \%$ \\
\hline $76 \mid$ ESP & $-0.0003 \%$ & $-0.0005 \%$ & $-0.0006 \%$ & $-0.0008 \%$ & $-0.0010 \%$ & $-0.0005 \%$ & $-0.0009 \%$ & $-0.0013 \%$ & $-0.0017 \%$ & $-0.0020 \%$ \\
\hline 77/SWE & $0.0001 \%$ & $-0.0001 \%$ & $-0.0004 \%$ & $-0.0006 \%$ & $-0.0008 \%$ & $0.0001 \%$ & $-0.0003 \%$ & $-0.0007 \%$ & $-0.0011 \%$ & $-0.0016 \%$ \\
\hline 78 GBR & $-0.0019 \%$ & $-0.0035 \%$ & $-0.0051 \%$ & $-0.0067 \%$ & $-0.0083 \%$ & $-0.0037 \%$ & $-0.0070 \%$ & $-0.0102 \%$ & $-0.0134 \%$ & $-0.0166 \%$ \\
\hline 79 CHE* & $0.0000 \%$ & $0.0000 \%$ & $0.0000 \%$ & $0.0000 \%$ & $0.0000 \%$ & $0.0000 \%$ & $0.0000 \%$ & $0.0000 \%$ & $0.0000 \%$ & $0.0000 \%$ \\
\hline ONOR & $-0.0038 \%$ & $-0.0088 \%$ & $-0.0139 \%$ & $-0.0190 \%$ & $-0.0240 \%$ & $-0.0075 \%$ & $-0.0176 \%$ & $-0.0278 \%$ & $-0.0379 \%$ & $-0.0481 \%$ \\
\hline 81 XEF & $0.0002 \%$ & $-0.0001 \%$ & $-0.0004 \%$ & $-0.0007 \%$ & $-0.0009 \%$ & $0.0003 \%$ & $-0.0002 \%$ & $-0.0008 \%$ & $-0.0013 \%$ & $-0.0019 \%$ \\
\hline ALB & $-0.0012 \%$ & $-0.0022 \%$ & $-0.0032 \%$ & $-0.0041 \%$ & $-0.0051 \%$ & $-0.0025 \%$ & $-0.0044 \%$ & $-0.0063 \%$ & $-0.0083 \%$ & $-0.0102 \%$ \\
\hline$B G R$ & -0.00 & $-0.0004 \%$ & $-0.0006 \%$ & $-0.0007 \%$ & $-0.0009 \%$ & -0.0 & -0.0 & $-0.0011 \%$ & & $-0.0018 \%$ \\
\hline $84 \mathrm{BLR}^{*}$ & $0.0000 \%$ & $0.0000 \%$ & $0.0000 \%$ & $0.0000 \%$ & $0.0000 \%$ & $0.0000 \%$ & $0.0000 \%$ & $0.0000 \%$ & $0.0000 \%$ & $0.0000 \%$ \\
\hline 85 HRV & $-0.0475 \%$ & $-0.0846 \%$ & $-0.1217 \%$ & $-0.1588 \%$ & $-0.1960 \%$ & $-0.0950 \%$ & $-0.1692 \%$ & $-0.2435 \%$ & $-0.3177 \%$ & $-0.3919 \%$ \\
\hline $86 \mid \mathrm{ROU}$ & $-0.0001 \%$ & $-0.0001 \%$ & $-0.0002 \%$ & $-0.0003 \%$ & $-0.0003 \%$ & $-0.0002 \%$ & $-0.0003 \%$ & $-0.0004 \%$ & $-0.0006 \%$ & $-0.0007 \%$ \\
\hline 87/RUS & $-0.0015 \%$ & $-0.0027 \%$ & $-0.0038 \%$ & $-0.0050 \%$ & $-0.0061 \%$ & $-0.0030 \%$ & $-0.0053 \%$ & $-0.0076 \%$ & $-0.0099 \%$ & $-0.0123 \%$ \\
\hline UKR & $-0.0004 \%$ & $-0.0007 \%$ & $-0.0011 \%$ & $-0.0014 \%$ & $-0.0017 \%$ & $-0.0008 \%$ & $-0.0015 \%$ & $-0.0022 \%$ & $-0.0028 \%$ & $-0.0035 \%$ \\
\hline 89 XEE* & $0.0000 \%$ & $0.0000 \%$ & $0.0000 \%$ & $0.0000 \%$ & $0.0000 \%$ & $0.0000 \%$ & $0.0000 \%$ & $0.0000 \%$ & $0.0000 \%$ & $0.0000 \%$ \\
\hline 90 XER & $-0.0005 \%$ & $-0.0010 \%$ & $-0.0014 \%$ & $-0.0018 \%$ & $-0.0022 \%$ & $-0.0011 \%$ & $-0.0019 \%$ & $-0.0028 \%$ & $-0.0036 \%$ & $-0.0045 \%$ \\
\hline $91 \mid \mathrm{KAZ}^{*}$ & $0.0000 \%$ & $0.0000 \%$ & $0.0000 \%$ & $0.0000 \%$ & $0.0000 \%$ & $0.0000 \%$ & $0.0000 \%$ & $0.0000 \%$ & $0.0000 \%$ & $0.0000 \%$ \\
\hline 92 KGZ* & $0.0000 \%$ & $0.0000 \%$ & $0.0000 \%$ & $0.0000 \%$ & $0.0000 \%$ & $0.0000 \%$ & 0.0 & $0.0000 \%$ & $0.0000 \%$ & $0.0000 \%$ \\
\hline 93 XSU* & $0.0000 \%$ & $0.0000 \%$ & $0.0000 \%$ & $0.0000 \%$ & $0.0000 \%$ & $0.0000 \%$ & $0 . C$ & $0.0000 \%$ & $0.0000 \%$ & $0.0000 \%$ \\
\hline 94 ARM* & $0.0000 \%$ & $0.0000 \%$ & $0.0000 \%$ & $0.0000 \%$ & $0.0000 \%$ & $0.0000 \%$ & $0.0000 \%$ & $0.0000 \%$ & $0.0000 \%$ & $0.0000 \%$ \\
\hline 95 AZE* & $0.0000 \%$ & $0.0000 \%$ & $0.0000 \%$ & $0.0000 \%$ & $0.0000 \%$ & $0.0000 \%$ & $0.0000 \%$ & $0.0000 \%$ & $0.0000 \%$ & $0.0000 \%$ \\
\hline 96GEO & $-0.0010 \%$ & $-0.0018 \%$ & $-0.0027 \%$ & $-0.0035 \%$ & $-0.0043 \%$ & $-0.0021 \%$ & $-0.0037 \%$ & $-0.0053 \%$ & $-0.0069 \%$ & $-0.0086 \%$ \\
\hline BHR & $-0.1932 \%$ & $-0.3442 \%$ & $-0.4952 \%$ & -0.64 & -0.7 & -0.3 & -0.6 & -0.5 & -1.2 & $-1.5945 \%$ \\
\hline $98 \mid \mathrm{IRN}$ & $-0.0003 \%$ & $-0.0007 \%$ & $-0.0011 \%$ & $-0.0015 \%$ & $-0.0019 \%$ & $-0.0006 \%$ & $-0.0014 \%$ & $-0.0022 \%$ & $-0.0030 \%$ & $-0.0037 \%$ \\
\hline $99 \mid$ ISR & $-0.0027 \%$ & $-0.0067 \%$ & $-0.0108 \%$ & $-0.0148 \%$ & $-0.0189 \%$ & $-0.0053 \%$ & $-0.0134 \%$ & $-0.0215 \%$ & $-0.0296 \%$ & $-0.0377 \%$ \\
\hline 100/JOR & $-0.0003 \%$ & -0.000 & $-0.0007 \%$ & -0.00 & -0.0 & -0.0 & $-0 . C$ & -0. & & $22 \%$ \\
\hline $101 \mid \mathrm{KWT}$ & $-0.0323 \%$ & $-0.0576 \%$ & $-0.0828 \%$ & $-0.1081 \%$ & $-0.1334 \%$ & $-0.0646 \%$ & $-0.1151 \%$ & $-0.1657 \%$ & $-0.2162 \%$ & $-0.2667 \%$ \\
\hline $102 \mathrm{OMN}$ & $-0.0105 \%$ & $-0.0196 \%$ & $-0.0286 \%$ & $-0.0377 \%$ & $-0.0467 \%$ & $-0.0210 \%$ & $-0.0391 \%$ & $-0.0572 \%$ & $-0.0753 \%$ & $-0.0934 \%$ \\
\hline 103 QAT & $-0.0834 \%$ & -0.14 & $-0.2138 \%$ & $-0.2790 \%$ & -0.34 & -0.1 & & & & $-0.6885 \%$ \\
\hline 104 SAU & $-0.0001 \%$ & $-0.0003 \%$ & $-0.0004 \%$ & $-0.0005 \%$ & $-0.0006 \%$ & $-0.0003 \%$ & $-0.0005 \%$ & $-0.0008 \%$ & $-0.0010 \%$ & $-0.0012 \%$ \\
\hline 106/TUR & $-0.0017 \%$ & $-0.0031 \%$ & $-0.0045 \%$ & $-0.0059 \%$ & $-0.0073 \%$ & $-0.0034 \%$ & $-0.0062 \%$ & $-0.0090 \%$ & $-0.0119 \%$ & $-0.0147 \%$ \\
\hline 105 ARE & $-0.0237 \%$ & $-0.0422 \%$ & $-0.0607 \%$ & $-0.0792 \%$ & $-0.0977 \%$ & $-0.0473 \%$ & $-0.0843 \%$ & $-0.1213 \%$ & $-0.1583 \%$ & $-0.1953 \%$ \\
\hline 107 XWS & -0.00 & -0.00 & $-0.0016 \%$ & -0.00 & $-0.0025 \%$ & -0.0 & -0.0 & -0.0 & -0.0 & $-0.0051 \%$ \\
\hline $108 \mathrm{EGY}$ & $-0.0076 \%$ & $-0.0136 \%$ & $-0.0196 \%$ & $-0.0256 \%$ & $-0.0316 \%$ & $-0.0152 \%$ & $-0.0272 \%$ & $-0.0392 \%$ & -0.05129 & $-0.0632 \%$ \\
\hline 109 MAR & $-0.0007 \%$ & $-0.0012 \%$ & $-0.0018 \%$ & $-0.0023 \%$ & $-0.0028 \%$ & $-0.0014 \%$ & $-0.0025 \%$ & $-0.0035 \%$ & -0.0 & $-0.0057 \%$ \\
\hline IDTUN & -0.0 & -0.0 & -0.0 & & -0.0 & & & & & $.06 \%$ \\
\hline XNF & $-0.0005 \%$ & -0.001 & $-0.0014 \%$ & $-0.0018 \%$ & $-0.0022 \%$ & $-0.0011 \%$ & -0.00 & $-0.0028 \%$ & $-0.0 c$ & $-0.0045 \%$ \\
\hline 112 BEN & $-0.0002 \%$ & $-0.0003 \%$ & $-0.0004 \%$ & $-0.0005 \%$ & $-0.0006 \%$ & $-0.0004 \%$ & $-0.0006 \%$ & $-0.0008 \%$ & $-0.0010 \%$ & $-0.0012 \%$ \\
\hline $113 \mathrm{BFA}^{*}$ & & & & & $0 . c$ & & & & & $0.0000 \%$ \\
\hline $114 \mid C M R$ & -0.000 & -0.00 & $-0.0008 \%$ & $-0.0 c$ & -0.0 & -0.00 & -0.0 & -0.0 & -0.0 & $-0.0025 \%$ \\
\hline $115 \mathrm{CIV}$ & $-0.0001 \%$ & $-0.0002 \%$ & $-0.0002 \%$ & $-0.0003 \%$ & $-0.0004 \%$ & $-0.0002 \%$ & $-0.0003 \%$ & $-0.0005 \%$ & $-0.0006 \%$ & $-0.0008 \%$ \\
\hline 116 GHA & -0.0 & -0 & -0.0 & -0.0 & -0.0 & -0.0 & $-0 . C$ & -0.0 & -0. & $-0.0010 \%$ \\
\hline GIN & -0.00 & -0.00 & $-0.0002 \%$ & $-0.0 c$ & -0.0 & -0.0 & -0.0 & -0.0 & -0.0 & $-0.0007 \%$ \\
\hline 118 NGA & $0.0000 \%$ & $-0.0001 \%$ & $-0.0001 \%$ & $-0.0001 \%$ & $-0.0002 \%$ & $-0.0001 \%$ & $-0.0001 \%$ & $-0.0002 \%$ & $-0.0003^{\circ}$ & $-0.0003 \%$ \\
\hline 119 SEN & $-0.0002 \%$ & $-0.0004 \%$ & $-0.0005 \%$ & -0.00 & -0.00 & $-0.0004 \%$ & $-0.0 c$ & $-0.0011 \%$ & -0.0 & $-0.0017 \%$ \\
\hline 0 TGO & & & & & & & & & & $05 \%$ \\
\hline $21 \times W F$ & -0.0 & -0.0 & $-0.0002 \%$ & -0.0 & -0.0 & -0.00 & -0.0 & -0.0 & -0.0 & $-0.0007 \%$ \\
\hline XCF & -0.0 & -0.00 & $-0.0 c$ & -0.0 & -0.0 & -0.0 & -0.0 & -0.0 & -0.0 & $-0.0012 \%$ \\
\hline XAC & & & -0.0 & & -0.0 & & & & & $.0013 \%$ \\
\hline $124 \mathrm{ETH}^{*}$ & $0 . c$ & 0.0 & 0.000 & $\%$ & $0 . c$ & 0 & 0 & 0.0 & 0.0000 & .0000 \\
\hline 125 KEN & $-0.0002 \%$ & $-0.0003 \%$ & $-0.0004 \%$ & $-0.0005 \%$ & $-0.0006 \%$ & $-0.0003 \%$ & $-0.0005 \%$ & $-0.0007 \%$ & $-0.0009 \%$ & $-0.0011 \%$ \\
\hline $126 \mathrm{MDG}$ & -0.0 & -0.0 & $-0.0027 \%$ & & $-0.0 c$ & -0.00 & & -0.0 & -0.0 & $87 \%$ \\
\hline 7 MWI* & & 0. & & & & & & & & 0.00 \\
\hline 128 MUS* & -0.0 & -0.031 & $-0.0448 \%$ & -0.05 & -0.0 & $9 \%$ & -0. & $95 \%$ & $59 \%$ & $1442 \%$ \\
\hline $129 / \mathrm{MOZ}$ & -0.00 & $-0.0008 \%$ & $-0.0012 \%$ & -0.00 & -0.0 & $-0.0009 \%$ & $-0.0016 \%$ & $-0.0023 \%$ & -0.0 & $-0.0037 \%$ \\
\hline $130 \mathrm{RWA}^{*}$ & & & $00 \%$ & & & & & & & $00 \%$ \\
\hline 131 TZA & $-0.0002 \%$ & $-0.0005 \%$ & $-0.0007 \%$ & $-0.0010 \%$ & $-0.0013 \%$ & $-0.0004 \%$ & $-0.0009 \%$ & $-0.0015 \%$ & $-0.0020 \%$ & $-0.0025 \%$ \\
\hline 132 UGA* & $0.0000 \%$ & $0.0000 \%$ & $0.0000 \%$ & $0.0000 \%$ & $0.0000 \%$ & $0.0000 \%$ & $0.0000 \%$ & $0.0000 \%$ & $0.0000 \%$ & $0.0000 \%$ \\
\hline 3 ZMB* & $0.0000 \%$ & $0.0000 \%$ & $0.0000 \%$ & $0.0000 \%$ & $0.0000 \%$ & $00 \%$ & $00 \%$ & 0.4 & & 00 \\
\hline 134|ZWE* & 0. & & $00 \%$ & $\%$ & & & & $0 \%$ & & $0.0000 \%$ \\
\hline $135 \mid$ XEC & $-0.0005 \%$ & $-0.0007 \%$ & $-0.0009 \%$ & $-0.0011 \%$ & $-0.0013 \%$ & $-0.0009 \%$ & $-0.0013 \%$ & $-0.0017 \%$ & $-0.0021 \%$ & $-0.0025 \%$ \\
\hline 6 BWA* & 0.0 & 0. & $0.0000 \%$ & $00 \%$ & $0.6-6$ & $00 \%$ & $0.0000 \%$ & 0.0000 & 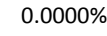 & 00 \\
\hline NAM & -0.00 & $-0.0006 \%$ & $-0.0009 \%$ & $-0.0012 \%$ & $-0.0015 \%$ & $-0.000 / \%$ & $-0.0013 \%$ & $-0.0019 \%$ & $-0.0024 \%$ & $-0.0030 \%$ \\
\hline ZA & & $-0.0005 \%$ & $-0.0007 \%$ & $-0.0009 \%$ & $-0.0011 \%$ & $-0.0005 \%$ & $-0.0009 \%$ & $-0.0013 \%$ & $-0.0017 \%$ & $-0.0021 \%$ \\
\hline XSC & 0.0 & $0.0000 \%$ & $0.0000 \%$ & $0.0000 \%$ & 0.0000 & $0.0000 \%$ & $0.0000^{\circ}$ & $0.0000 \%$ & $0.0000 \%$ & 0.0000 \\
\hline $140 \times \mathrm{XTW}$ & & & $8 \%$ & & & & & & & \\
\hline
\end{tabular}


Table A3-1. Agriculture: percentage variation in multi-factor productivity. Values lower than $-10 \%$ in red.

\begin{tabular}{|c|c|c|c|c|c|c|c|c|c|c|c|c|c|c|c|}
\hline \multirow[b]{2}{*}{ Code } & \multicolumn{5}{|c|}{ MAIZE } & \multicolumn{5}{|c|}{ WHEAT } & \multicolumn{5}{|c|}{ RICE } \\
\hline & $+1^{\circ} \mathrm{C}$ & $+2^{\circ} \mathrm{C}$ & $+3^{\circ} \mathrm{C}$ & $+4^{\circ} \mathrm{C}$ & $+5^{\circ} \mathrm{C}$ & $+1^{\circ} \mathrm{C}$ & $+2^{\circ} \mathrm{C}$ & $+3^{\circ} \mathrm{C}$ & $+4^{\circ} \mathrm{C}$ & $+5^{\circ} \mathrm{C}$ & $+1^{\circ} \mathrm{C}$ & $+2^{\circ} \mathrm{C}$ & $+3^{\circ} \mathrm{C}$ & $+4^{\circ} \mathrm{C}$ & $+5^{\circ} \mathrm{C}$ \\
\hline 1 AUS & $-1.94 \%$ & $-4.56 \%$ & $-5.88 \%$ & $-8.56 \%$ & $-11.94 \%$ & $-2.19 \%$ & $-5.38 \%$ & $-11.06 \%$ & $-16.13 \%$ & $-19.94 \%$ & $-2.75 \%$ & $-2.69 \%$ & $-2.63 \%$ & $-6.69 \%$ & $-13.50 \%$ \\
\hline 2 NZL & $-0.78 \%$ & $-2.63 \%$ & $-3.55 \%$ & $-6.63 \%$ & $-10.78 \%$ & $-5.68 \%$ & $-6.15 \%$ & $-6.03 \%$ & $-6.05 \%$ & $-6.38 \%$ & $-4.30 \%$ & $-3.08 \%$ & $-1.85 \%$ & $-7.08 \%$ & $-16.60 \%$ \\
\hline $3 \mathrm{XOC}$ & $-3.03 \%$ & $-6.38 \%$ & $-8.05 \%$ & $-10.38 \%$ & $-13.03 \%$ & $1.08 \%$ & $-4.65 \%$ & $-15.78 \%$ & $-25.55 \%$ & $-32.63 \%$ & $-1.30 \%$ & $-2.33 \%$ & $-3.35 \%$ & $-6.33 \%$ & $-10.60 \%$ \\
\hline $4 \mathrm{CHN}$ & $-1.34 \%$ & $-3.56 \%$ & $-4.68 \%$ & $-7.56 \%$ & $-11.34 \%$ & $-3.99 \%$ & $-5.78 \%$ & $-8.46 \%$ & $-10.93 \%$ & $-12.94 \%$ & $-3.55 \%$ & $-2.89 \%$ & $-2.23 \%$ & $-6.89 \%$ & $-15.10 \%$ \\
\hline 5 HKG & $-2.35 \%$ & $-5.25 \%$ & $-6.70 \%$ & $-9.25 \%$ & $-12.35 \%$ & $-0.95 \%$ & $-5.10 \%$ & $-12.85 \%$ & $-19.70 \%$ & $-24.75 \%$ & $-2.20 \%$ & $-2.55 \%$ & $-2.90 \%$ & $-6.55 \%$ & $-12.40 \%$ \\
\hline $6 \mathrm{JPN}$ & $-1.49 \%$ & $-3.81 \%$ & $-4.98 \%$ & $-7.81 \%$ & $-11.49 \%$ & $-3.54 \%$ & $-5.68 \%$ & $-9.11 \%$ & $-12.23 \%$ & $-14.69 \%$ & $-3.35 \%$ & $-2.84 \%$ & $-2.33 \%$ & $-6.84 \%$ & $-14.70 \%$ \\
\hline 7 KOR & $-1.34 \%$ & $-3.56 \%$ & $-4.68 \%$ & $-7.56 \%$ & $-11.34 \%$ & $-3.99 \%$ & $-5.78 \%$ & $-8.46 \%$ & $-10.93 \%$ & $-12.94 \%$ & $-3.55 \%$ & $-2.89 \%$ & $-2.23 \%$ & $-6.89 \%$ & $-15.10 \%$ \\
\hline 8 MNG & $-0.48 \%$ & $-2.13 \%$ & $-2.95 \%$ & $-6.13 \%$ & $-10.48 \%$ & $-6.58 \%$ & $-6.35 \%$ & $-4.73 \%$ & $-3.45 \%$ & $-2.88 \%$ & $-4.70 \%$ & $-3.18 \%$ & $-1.65 \%$ & $-7.18 \%$ & $-17.40 \%$ \\
\hline 9 TWN & $-2.20 \%$ & $-5.00 \%$ & $-6.40 \%$ & $-9.00 \%$ & $-12.20 \%$ & $-1.40 \%$ & $-5.20 \%$ & $-12.20 \%$ & $-18.40 \%$ & $-23.00 \%$ & $-2.40 \%$ & $-2.60 \%$ & $-2.80 \%$ & $-6.60 \%$ & $-12.80 \%$ \\
\hline 10XEA & $-3.06 \%$ & $-6.44 \%$ & $-8.13 \%$ & $-10.44 \%$ & $-13.06 \%$ & $1.19 \%$ & $-4.63 \%$ & $-15.94 \%$ & $-25.88 \%$ & $-33.06 \%$ & $-1.25 \%$ & $-2.31 \%$ & $-3.38 \%$ & $-6.31 \%$ & $-10.50 \%$ \\
\hline 11 BRN & $-3.66 \%$ & $-7.44 \%$ & $-9.33 \%$ & $-11.44 \%$ & $-13.66 \%$ & $2.99 \%$ & $-4.23 \%$ & $-18.54 \%$ & $-31.08 \%$ & $-40.06 \%$ & $-0.45 \%$ & $-2.11 \%$ & $-3.78 \%$ & $-6.11 \%$ & $-8.90 \%$ \\
\hline $12 \mathrm{KHM}$ & $-3.06 \%$ & $-6.44 \%$ & $-8.13 \%$ & $-10.44 \%$ & $-13.06 \%$ & $1.19 \%$ & $-4.63 \%$ & $-15.94 \%$ & $-25.88 \%$ & $-33.06 \%$ & $-1.25 \%$ & $-2.31 \%$ & $-3.38 \%$ & $-6.31 \%$ & $-10.50 \%$ \\
\hline 13 IDN & $-3.81 \%$ & $-7.69 \%$ & $-9.63 \%$ & $-11.69 \%$ & $-13.81 \%$ & $3.44 \%$ & $-4.13 \%$ & $-19.19 \%$ & $-32.38 \%$ & $-41.81 \%$ & $-0.25 \%$ & $-2.06 \%$ & $-3.88 \%$ & $-6.06 \%$ & $-8.50 \%$ \\
\hline 14 LAO & $-2.61 \%$ & $-5.69 \%$ & $-7.23 \%$ & $-9.69 \%$ & $-12.61 \%$ & $-0.16 \%$ & $-4.93 \%$ & $-13.99 \%$ & $-21.98 \%$ & $-27.81 \%$ & $-1.85 \%$ & $-2.46 \%$ & $-3.08 \%$ & $-6.46 \%$ & $-11.70 \%$ \\
\hline 15 MYS & $-3.66 \%$ & $-7.44 \%$ & $-9.33 \%$ & $-11.44 \%$ & $-13.66 \%$ & $2.99 \%$ & $-4.23 \%$ & $-18.54 \%$ & $-31.08 \%$ & $-40.06 \%$ & $-0.45 \%$ & $-2.11 \%$ & $-3.78 \%$ & $-6.11 \%$ & $-8.90 \%$ \\
\hline 16 PHL & $-3.03 \%$ & $-6.38 \%$ & $-8.05 \%$ & $-10.38 \%$ & $-13.03 \%$ & $1.08 \%$ & $-4.65 \%$ & $-15.78 \%$ & $-25.55 \%$ & $-32.63 \%$ & $-1.30 \%$ & $-2.33 \%$ & $-3.35 \%$ & $-6.33 \%$ & $-10.60 \%$ \\
\hline 17 SGP & $-3.93 \%$ & $-7.88 \%$ & $-9.85 \%$ & $-11.88 \%$ & $-13.93 \%$ & $3.78 \%$ & $-4.05 \%$ & $-19.68 \%$ & $-33.35 \%$ & $-43.13 \%$ & $-0.10 \%$ & $-2.03 \%$ & $-3.95 \%$ & $-6.03 \%$ & $-8.20 \%$ \\
\hline 18 THA & $-3.06 \%$ & $-6.44 \%$ & $-8.13 \%$ & $-10.44 \%$ & $-13.06 \%$ & $1.19 \%$ & $-4.63 \%$ & $-15.94 \%$ & $-25.88 \%$ & $-33.06 \%$ & $-1.25 \%$ & $-2.31 \%$ & $-3.38 \%$ & $-6.31 \%$ & $-10.50 \%$ \\
\hline 19 VNM & $-2.84 \%$ & $-6.06 \%$ & $-7.68 \%$ & $-10.06 \%$ & $-12.84 \%$ & $0.51 \%$ & $-4.78 \%$ & $-14.96 \%$ & $-23.93 \%$ & $-30.44 \%$ & $-1.55 \%$ & $-2.39 \%$ & $-3.23 \%$ & $-6.39 \%$ & $-11.10 \%$ \\
\hline 20 XSE & $-3.06 \%$ & $-6.44 \%$ & $-8.13 \%$ & $-10.44 \%$ & $-13.06 \%$ & $1.19 \%$ & $-4.63 \%$ & $-15.94 \%$ & $-25.88 \%$ & $-33.06 \%$ & $-1.25 \%$ & $-2.31 \%$ & $-3.38 \%$ & $-6.31 \%$ & $-10.50 \%$ \\
\hline $21 \mid B G D$ & $-2.20 \%$ & $-5.00 \%$ & $-6.40 \%$ & $-9.00 \%$ & $-12.20 \%$ & $-1.40 \%$ & $-5.20 \%$ & $-12.20 \%$ & $-18.40 \%$ & $-23.00 \%$ & $-2.40 \%$ & $-2.60 \%$ & $-2.80 \%$ & $-6.60 \%$ & $-12.80 \%$ \\
\hline 22 IND & $-2.31 \%$ & $-5.19 \%$ & $-6.63 \%$ & $-9.19 \%$ & $-12.31 \%$ & $-1.06 \%$ & $-5.13 \%$ & $-12.69 \%$ & $-19.38 \%$ & $-24.31 \%$ & $-2.25 \%$ & $-2.56 \%$ & $-2.88 \%$ & $-6.56 \%$ & $-12.50 \%$ \\
\hline $23 \mathrm{NPL}$ & $-1.90 \%$ & $-4.50 \%$ & $-5.80 \%$ & $-8.50 \%$ & $-11.90 \%$ & $-2.30 \%$ & $-5.40 \%$ & $-10.90 \%$ & $-15.80 \%$ & $-19.50 \%$ & $-2.80 \%$ & $-2.70 \%$ & $-2.60 \%$ & $-6.70 \%$ & $-13.60 \%$ \\
\hline $24 \mid$ PAK & $-1.75 \%$ & $-4.25 \%$ & $-5.50 \%$ & $-8.25 \%$ & $-11.75 \%$ & $-2.75 \%$ & $-5.50 \%$ & $-10.25 \%$ & $-14.50 \%$ & $-17.75 \%$ & $-3.00 \%$ & $-2.75 \%$ & $-2.50 \%$ & $-6.75 \%$ & $-14.00 \%$ \\
\hline 25 LKA & $-3.40 \%$ & $-7.00 \%$ & $-8.80 \%$ & $-11.00 \%$ & $-13.40 \%$ & $2.20 \%$ & $-4.40 \%$ & $-17.40 \%$ & $-28.80 \%$ & $-37.00 \%$ & $-0.80 \%$ & $-2.20 \%$ & $-3.60 \%$ & $-6.20 \%$ & $-9.60 \%$ \\
\hline 26 XSA & $-2.31 \%$ & $-5.19 \%$ & $-6.63 \%$ & $-9.19 \%$ & $-12.31 \%$ & $-1.06 \%$ & $-5.13 \%$ & $-12.69 \%$ & $-19.38 \%$ & $-24.31 \%$ & $-2.25 \%$ & $-2.56 \%$ & -2.8 & $-6.56 \%$ & $-12.50 \%$ \\
\hline 27 CAN & $0.69 \%$ & $-0.19 \%$ & $-0.63 \%$ & $-4.19 \%$ & $-9.31 \%$ & $-10.06 \%$ & $-7.13 \%$ & $0.31 \%$ & $6.63 \%$ & $10.69 \%$ & $-6.25 \%$ & $-3.56 \%$ & $-0.88 \%$ & $-7.56 \%$ & $-20.50 \%$ \\
\hline 28 USA & $-1.23 \%$ & $-3.38 \%$ & $-4.45 \%$ & $-7.38 \%$ & $-11.23 \%$ & $-4.33 \%$ & $-5.85 \%$ & $-7.98 \%$ & $-9.95 \%$ & $-11.63 \%$ & $-3.70 \%$ & $-2.93 \%$ & $-2.15 \%$ & $-6.93 \%$ & $-15.40 \%$ \\
\hline 29 MEX & $-2.20 \%$ & $-5.00 \%$ & $-6.40 \%$ & $-9.00 \%$ & $-12.20 \%$ & $-1.40 \%$ & $-5.20 \%$ & $-12.20 \%$ & $-18.40 \%$ & $-23.00 \%$ & $-2.40 \%$ & $-2.60 \%$ & $-2.80 \%$ & $-6.60 \%$ & $-12.80 \%$ \\
\hline 30XNA & $-1.23 \%$ & $-3.38 \%$ & $-4.45 \%$ & $-7.38 \%$ & $-11.23 \%$ & $-4.33 \%$ & $-5.85 \%$ & $-7.98 \%$ & $-9.95 \%$ & $-11.63 \%$ & $-3.70 \%$ & $-2.93 \%$ & $-2.15 \%$ & $-6.93 \%$ & $-15.40 \%$ \\
\hline 31 ARG & $-1.11 \%$ & $-3.19 \%$ & $-4.23 \%$ & $-7.19 \%$ & $-11.11 \%$ & $-4.66 \%$ & $-5.93 \%$ & $-7.49 \%$ & $-8.98 \%$ & $-10.31 \%$ & $-3.85 \%$ & $-2.96 \%$ & $-2.08 \%$ & $-6.96 \%$ & $-15.70 \%$ \\
\hline $32 \mathrm{BOL}$ & $-2.76 \%$ & $-5.94 \%$ & $-7.53 \%$ & $-9.94 \%$ & $-12.76 \%$ & $0.29 \%$ & $-4.83 \%$ & $-14.64 \%$ & $-23.28 \%$ & $-29.56 \%$ & $-1.65 \%$ & $-2.41 \%$ & $-3.18 \%$ & $-6.41 \%$ & $-11.30 \%$ \\
\hline 33 BRA & $-2.54 \%$ & $-5.56 \%$ & $-7.08 \%$ & $-9.56 \%$ & $-12.54 \%$ & $-0.39 \%$ & $-4.98 \%$ & $-13.66 \%$ & $-21.33 \%$ & $-26.94 \%$ & $-1.95 \%$ & $-2.49 \%$ & $-3.03 \%$ & $-6.49 \%$ & $-11.90 \%$ \\
\hline $34 \mathrm{CHL}$ & $-1.26 \%$ & $-3.44 \%$ & $-4.53 \%$ & $-7.44 \%$ & $-11.26 \%$ & $-4.21 \%$ & $-5.83 \%$ & $-8.14 \%$ & $-10.28 \%$ & $-12.06 \%$ & $-3.65 \%$ & $-2.91 \%$ & $-2.18 \%$ & $-6.91 \%$ & $-15.30 \%$ \\
\hline $35 \mathrm{COL}$ & $-3.70 \%$ & $-7.50 \%$ & $-9.40 \%$ & $-11.50 \%$ & $-13.70 \%$ & $3.10 \%$ & $-4.20 \%$ & $-18.70 \%$ & $-31.40 \%$ & $-40.50 \%$ & $-0.40 \%$ & $-2.10 \%$ & $-3.80 \%$ & $-6.10 \%$ & $-8.80 \%$ \\
\hline 36 ECU & $-3.89 \%$ & $-7.81 \%$ & $-9.78 \%$ & $-11.81 \%$ & $-13.89 \%$ & $3.66 \%$ & $-4.08 \%$ & $-19.51 \%$ & $-33.03 \%$ & $-42.69 \%$ & $-0.15 \%$ & $-2.04 \%$ & $-3.93 \%$ & $-6.04 \%$ & $-8.30 \%$ \\
\hline 37 PRY & $-2.28 \%$ & $-5.13 \%$ & $-6.55 \%$ & $-9.13 \%$ & $-12.28 \%$ & $-1.18 \%$ & $-5.15 \%$ & $-12.53 \%$ & $-19.05 \%$ & $-23.88 \%$ & $-2.30 \%$ & $-2.58 \%$ & $-2.85 \%$ & $-6.58 \%$ & $-12.60 \%$ \\
\hline 38 PER & $-3.33 \%$ & $-6.88 \%$ & $-8.65 \%$ & $-10.88 \%$ & $-13.33 \%$ & $1.98 \%$ & $-4.45 \%$ & $-17.08 \%$ & $-28.15 \%$ & $-36.13 \%$ & $-0.90 \%$ & $-2.23 \%$ & $-3.55 \%$ & $-6.23 \%$ & $-9.80 \%$ \\
\hline 39 URY & $-1.56 \%$ & $-3.94 \%$ & $-5.13 \%$ & $-7.94 \%$ & $-11.56 \%$ & $-3.31 \%$ & $-5.63 \%$ & $-9.44 \%$ & $-12.88 \%$ & $-15.56 \%$ & $-3.25 \%$ & $-2.81 \%$ & $-2.38 \%$ & $-6.81 \%$ & $-14.50 \%$ \\
\hline 40 VEN & $-3.51 \%$ & $-7.19 \%$ & $-9.03 \%$ & $-11.19 \%$ & $-13.51 \%$ & $2.54 \%$ & $-4.33 \%$ & $-17.89 \%$ & $-29.78 \%$ & $-38.31 \%$ & $-0.65 \%$ & $-2.16 \%$ & $-3.68 \%$ & $-6.16 \%$ & $-9.30 \%$ \\
\hline 41 XSM & $-1.56 \%$ & $-3.94 \%$ & $-5.13 \%$ & $-7.94 \%$ & $-11.56 \%$ & $-3.31 \%$ & $-5.63 \%$ & $-9.44 \%$ & $-12.88 \%$ & $-15.56 \%$ & $-3.25 \%$ & $-2.81 \%$ & $-2.38 \%$ & $-6.81 \%$ & $-14.50 \%$ \\
\hline 42 CRI & $-3.29 \%$ & $-6.81 \%$ & $-8.58 \%$ & $-10.81 \%$ & $-13.29 \%$ & $1.86 \%$ & $-4.48 \%$ & $-16.91 \%$ & $-27.83 \%$ & $-35.69 \%$ & $-0.95 \%$ & $-2.24 \%$ & $-3.53 \%$ & $-6.24 \%$ & $-9.90 \%$ \\
\hline 43 GTM & $-2.80 \%$ & $-6.00 \%$ & $-7.60 \%$ & $-10.00 \%$ & $-12.80 \%$ & $0.40 \%$ & $-4.80 \%$ & $-14.80 \%$ & $-23.60 \%$ & $-30.00 \%$ & $-1.60 \%$ & $-2.40 \%$ & $-3.20 \%$ & $-6.40 \%$ & $-11.20 \%$ \\
\hline 44 HND & $-2.91 \%$ & $-6.19 \%$ & $-7.83 \%$ & $-10.19 \%$ & $-12.91 \%$ & $0.74 \%$ & $-4.73 \%$ & $-15.29 \%$ & $-24.58 \%$ & $-31.31 \%$ & $-1.45 \%$ & $-2.36 \%$ & $-3.28 \%$ & $-6.36 \%$ & $-10.90 \%$ \\
\hline 45 NIC & $-3.03 \%$ & $-6.38 \%$ & $-8.05 \%$ & $-10.38 \%$ & $-13.03 \%$ & $1.08 \%$ & $-4.65 \%$ & $-15.78 \%$ & $-25.55 \%$ & $-32.63 \%$ & $-1.30 \%$ & $-2.33 \%$ & $-3.35 \%$ & $-6.33 \%$ & $-10.60 \%$ \\
\hline 46 SLV & $-3.36 \%$ & $-6.94 \%$ & $-8.73 \%$ & $-10.94 \%$ & $-13.36 \%$ & $2.09 \%$ & $-4.43 \%$ & $-17.24 \%$ & $-28.48 \%$ & $-36.56 \%$ & $-0.85 \%$ & $-2.21 \%$ & $-3.58 \%$ & $-6.21 \%$ & $-9.70 \%$ \\
\hline 47 PAN & $-2.99 \%$ & $-6.31 \%$ & $-7.98 \%$ & $-10.31 \%$ & $-12.99 \%$ & $0.96 \%$ & $-4.68 \%$ & $-15.61 \%$ & $-25.23 \%$ & $-32.19 \%$ & $-1.35 \%$ & $-2.34 \%$ & $-3.33 \%$ & $-6.34 \%$ & $-10.70 \%$ \\
\hline $48 \times C A$ & $-3.03 \%$ & $-6.38 \%$ & $-8.05 \%$ & $-10.38 \%$ & $-13.03 \%$ & $1.08 \%$ & $-4.65 \%$ & $-15.78 \%$ & $-25.55 \%$ & $-32.63 \%$ & $-1.30 \%$ & $-2.33 \%$ & $-3.35 \%$ & -6.3 & $-10.60 \%$ \\
\hline 49 DOM & $-2.58 \%$ & $-5.63 \%$ & $-7.15 \%$ & $-9.63 \%$ & $-12.58 \%$ & $-0.28 \%$ & $-4.95 \%$ & $-13.83 \%$ & $-21.65 \%$ & $-27.38 \%$ & $-1.90 \%$ & $-2.48 \%$ & $-3.05 \%$ & $-6.48 \%$ & $-11.80 \%$ \\
\hline 50 JAM & $-2.65 \%$ & $-5.75 \%$ & $-7.30 \%$ & $-9.75 \%$ & $-12.65 \%$ & $-0.05 \%$ & $-4.90 \%$ & $-14.15 \%$ & $-22.30 \%$ & $-28.25 \%$ & $-1.80 \%$ & $-2.45 \%$ & $-3.10 \%$ & $-6.45 \%$ & $-11.60 \%$ \\
\hline 51 PRI & $-2.65 \%$ & $-5.75 \%$ & $-7.30 \%$ & $-9.75 \%$ & $-12.65 \%$ & $-0.05 \%$ & $-4.90 \%$ & $-14.15 \%$ & $-22.30 \%$ & $-28.25 \%$ & $-1.80 \%$ & $-2.45 \%$ & $-3.10 \%$ & $-6.45 \%$ & $-11.60 \%$ \\
\hline 52 & $-3.18 \%$ & $-6.63 \%$ & $-8.35 \%$ & $-10.63 \%$ & $-13.18 \%$ & $1.53 \%$ & $-4.55 \%$ & $-16.43 \%$ & $-26.85 \%$ & $-34.38 \%$ & $-1.10 \%$ & $-2.28 \%$ & $-3.45 \%$ & $-6.28 \%$ & $-10.20 \%$ \\
\hline $53 \mid X C B$ & $-2.65 \%$ & $-5.75 \%$ & $-7.30 \%$ & $-9.75 \%$ & $-12.65 \%$ & $-0.05 \%$ & $-4.90 \%$ & $-14.15 \%$ & $-22.30 \%$ & $-28.25 \%$ & $-1.80 \%$ & $-2.45 \%$ & $-3.10 \%$ & $-6.45 \%$ & $-11.60 \%$ \\
\hline 54 AUT & $-0.44 \%$ & $-2.06 \%$ & $-2.88 \%$ & $-6.06 \%$ & $-10.44 \%$ & $-6.69 \%$ & $-6.38 \%$ & $-4.56 \%$ & $-3.13 \%$ & $-2.44 \%$ & $-4.75 \%$ & $-3.19 \%$ & $-1.63 \%$ & $-7.19 \%$ & $-17.50 \%$ \\
\hline $55 \mathrm{BEL}$ & $-0.21 \%$ & $-1.69 \%$ & $-2.43 \%$ & $-5.69 \%$ & $-10.21 \%$ & $-7.36 \%$ & $-6.53 \%$ & $-3.59 \%$ & $-1.18 \%$ & $0.19 \%$ & $-5.05 \%$ & $-3.26 \%$ & $-1.48 \%$ & $-7.26 \%$ & $-18.10 \%$ \\
\hline 56 CYP & $-1.38 \%$ & $-3.63 \%$ & $-4.75 \%$ & $-7.63 \%$ & $-11.38 \%$ & $-3.88 \%$ & $-5.75 \%$ & $-8.63 \%$ & $-11.25 \%$ & $-13.38 \%$ & $-3.50 \%$ & $-2.88 \%$ & $-2.25 \%$ & $-6.88 \%$ & $-15.00 \%$ \\
\hline 57 CZE & $-0.25 \%$ & $-1.75 \%$ & $-2.50 \%$ & $-5.75 \%$ & $-10.25 \%$ & $-7.25 \%$ & $-6.50 \%$ & $-3.75 \%$ & $-1.50 \%$ & $-0.25 \%$ & $-5.00 \%$ & $-3.25 \%$ & $-1.50 \%$ & $-7.25 \%$ & $-18.00 \%$ \\
\hline 58 DNK & $0.24 \%$ & $-0.94 \%$ & $-1.53 \%$ & $-4.94 \%$ & $-9.76 \%$ & $-8.71 \%$ & $-6.83 \%$ & $-1.64 \%$ & $2.73 \%$ & $5.44 \%$ & $-5.65 \%$ & $-3.41 \%$ & $-1.18 \%$ & $-7.41 \%$ & $-19.30 \%$ \\
\hline 59 EST & $0.43 \%$ & $-0.63 \%$ & $-1.15 \%$ & $-4.63 \%$ & $-9.58 \%$ & $-9.28 \%$ & $-6.95 \%$ & $-0.82 \%$ & $4.35 \%$ & $7.63 \%$ & $-5.90 \%$ & $-3.48 \%$ & $-1.05 \%$ & $-7.48 \%$ & $-19.80 \%$ \\
\hline 60 FIN & $0.88 \%$ & $0.13 \%$ & $-0.25 \%$ & $-3.88 \%$ & $-9.13 \%$ & $-10.63 \%$ & $-7.25 \%$ & $1.13 \%$ & $8.25 \%$ & $12.88 \%$ & $-6.50 \%$ & $-3.63 \%$ & $-0.75 \%$ & $-7.63 \%$ & $-21.00 \%$ \\
\hline 61 FRA & $-0.51 \%$ & $-2.19 \%$ & $-3.03 \%$ & $-6.19 \%$ & $-10.51 \%$ & $-6.46 \%$ & $-6.33 \%$ & $-4.89 \%$ & $-3.78 \%$ & $-3.31 \%$ & $-4.65 \%$ & $-3.16 \%$ & $-1.68 \%$ & $-7.16 \%$ & $-17.30 \%$ \\
\hline 62 DEU & $-0.18 \%$ & $-1.63 \%$ & $-2.35 \%$ & $-5.63 \%$ & $-10.18 \%$ & $-7.48 \%$ & $-6.55 \%$ & $-3.43 \%$ & $-0.85 \%$ & $0.63 \%$ & $-5.10 \%$ & $-3.28 \%$ & $-1.45 \%$ & $-7.28 \%$ & $-18.20 \%$ \\
\hline 63 GRC & $-1.11 \%$ & $-3.19 \%$ & $-4.23 \%$ & $-7.19 \%$ & $-11.11 \%$ & $-4.66 \%$ & $-5.93 \%$ & $-7.49 \%$ & $-8.98 \%$ & $-10.31 \%$ & $-3.85 \%$ & $-2.96 \%$ & $-2.08 \%$ & $-6.96 \%$ & $-15.70 \%$ \\
\hline 64 HUN & $-0.44 \%$ & $-2.06 \%$ & $-2.88 \%$ & $-6.06 \%$ & $-10.44 \%$ & $-6.69 \%$ & $-6.38 \%$ & $-4.56 \%$ & $-3.13 \%$ & $-2.44 \%$ & $-4.75 \%$ & $-3.19 \%$ & $-1.63 \%$ & $-7.19 \%$ & $-17.50 \%$ \\
\hline $65 \mid \mathrm{IRL}$ & $-0.02 \%$ & $-1.38 \%$ & $-2.05 \%$ & $-5.38 \%$ & $-10.03 \%$ & $-7.93 \%$ & $-6.65 \%$ & $-2.78 \%$ & $0.45 \%$ & $2.38 \%$ & $-5.30 \%$ & $-3.33 \%$ & $-1.35 \%$ & $-7.33 \%$ & $-18.60 \%$ \\
\hline 66 ITA & $-0.89 \%$ & $-2.81 \%$ & $-3.78 \%$ & $-6.81 \%$ & $-10.89 \%$ & $-5.34 \%$ & $-6.08 \%$ & $-6.51 \%$ & $-7.03 \%$ & $-7.69 \%$ & $-4.15 \%$ & $-3.04 \%$ & $-1.93 \%$ & $-7.04 \%$ & $-16.30 \%$ \\
\hline 67 LVA & $0.24 \%$ & $-0.94 \%$ & $-1.53 \%$ & $-4.94 \%$ & $-9.76 \%$ & $-8.71 \%$ & $-6.83 \%$ & $-1.64 \%$ & $2.73 \%$ & $5.44 \%$ & $-5.65 \%$ & $-3.41 \%$ & $-1.18 \%$ & $-7.41 \%$ & $-19.30 \%$ \\
\hline 68 LTU & $0.13 \%$ & $-1.13 \%$ & $-1.75 \%$ & $-5.13 \%$ & $-9.88 \%$ & $-8.38 \%$ & $-6.75 \%$ & $-2.13 \%$ & $1.75 \%$ & $4.13 \%$ & $-5.50 \%$ & $-3.38 \%$ & $-1.25 \%$ & $-7.38 \%$ & $-19.00 \%$ \\
\hline 69 LUX & $-0.29 \%$ & $-1.81 \%$ & $-2.58 \%$ & $-5.81 \%$ & $-10.29 \%$ & $-7.14 \%$ & $-6.48 \%$ & $-3.91 \%$ & $-1.83 \%$ & $-0.69 \%$ & $-4.95 \%$ & $-3.24 \%$ & $-1.53 \%$ & $-7.24 \%$ & $-17.90 \%$ \\
\hline 70 MLT & $-1.34 \%$ & $-3.56 \%$ & $-4.68 \%$ & $-7.56 \%$ & $-11.34 \%$ & $-3.99 \%$ & $-5.78 \%$ & $-8.46 \%$ & $-10.93 \%$ & $-12.94 \%$ & $-3.55 \%$ & $-2.89 \%$ & $-2.23 \%$ & $-6.89 \%$ & $-15.10 \%$ \\
\hline
\end{tabular}


Table A3-2. Agriculture: percentage variation in multi-factor productivity. Values lower than $-10 \%$ in red.

\begin{tabular}{|c|c|c|c|c|c|c|c|c|c|c|c|c|c|c|c|}
\hline \multirow[b]{2}{*}{ Code } & \multicolumn{5}{|c|}{ MAIZE } & \multicolumn{5}{|c|}{ WHEAT } & \multicolumn{5}{|c|}{ RICE } \\
\hline & $+1^{\circ} \mathrm{C}$ & $+2^{\circ} \mathrm{C}$ & $+3^{\circ} \mathrm{C}$ & $+4^{\circ} \mathrm{C}$ & $+5^{\circ} \mathrm{C}$ & $+1^{\circ} \mathrm{C}$ & $+2^{\circ} \mathrm{C}$ & $+3^{\circ} \mathrm{C}$ & $+4^{\circ} \mathrm{C}$ & $+5^{\circ} \mathrm{C}$ & $+1^{\circ} \mathrm{C}$ & $+2^{\circ} \mathrm{C}$ & $+3^{\circ} \mathrm{C}$ & $+4^{\circ} \mathrm{C}$ & $+5^{\circ} \mathrm{C}$ \\
\hline $71 \mid$ NLD & $-0.06 \%$ & $-1.44 \%$ & $-2.13 \%$ & $-5.44 \%$ & $-10.06 \%$ & $-7.81 \%$ & $-6.63 \%$ & $-2.94 \%$ & $0.13 \%$ & $1.94 \%$ & $-5.25 \%$ & $-3.31 \%$ & $-1.38 \%$ & $-7.31 \%$ & $-18.50 \%$ \\
\hline $72 \mid \mathrm{POL}$ & $-0.10 \%$ & $-1.50 \%$ & $-2.20 \%$ & $-5.50 \%$ & $-10.10 \%$ & $-7.70 \%$ & $-6.60 \%$ & $-3.10 \%$ & $-0.20 \%$ & $1.50 \%$ & $-5.20 \%$ & $-3.30 \%$ & $-1.40 \%$ & $-7.30 \%$ & $-18.40 \%$ \\
\hline 73 PRT & $-1.04 \%$ & $-3.06 \%$ & $-4.08 \%$ & $-7.06 \%$ & $-11.04 \%$ & $-4.89 \%$ & $-5.98 \%$ & $-7.16 \%$ & $-8.33 \%$ & $-9.44 \%$ & $-3.95 \%$ & $-2.99 \%$ & $-2.03 \%$ & $-6.99 \%$ & $-15.90 \%$ \\
\hline 74 SVK & $-0.33 \%$ & $-1.88 \%$ & $-2.65 \%$ & $-5.88 \%$ & $-10.33 \%$ & $-7.03 \%$ & $-6.45 \%$ & $-4.08 \%$ & $-2.15 \%$ & $-1.13 \%$ & $-4.90 \%$ & $-3.23 \%$ & $-1.55 \%$ & $-7.23 \%$ & $-17.80 \%$ \\
\hline 75 SVN & $-0.55 \%$ & $-2.25 \%$ & $-3.10 \%$ & $-6.25 \%$ & $-10.55 \%$ & $-6.35 \%$ & $-6.30 \%$ & $-5.05 \%$ & $-4.10 \%$ & $-3.75 \%$ & $-4.60 \%$ & $-3.15 \%$ & $-1.70 \%$ & $-7.15 \%$ & $-17.20 \%$ \\
\hline 76 ESP & $-1.00 \%$ & $-3.00 \%$ & $-4.00 \%$ & $-7.00 \%$ & $-11.00 \%$ & $-5.00 \%$ & $-6.00 \%$ & $-7.00 \%$ & $-8.00 \%$ & $-9.00 \%$ & $-4.00 \%$ & $-3.00 \%$ & $-2.00 \%$ & $-7.00 \%$ & $-16.00 \%$ \\
\hline 77)SWE & $0.65 \%$ & $-0.25 \%$ & $-0.70 \%$ & $-4.25 \%$ & $-9.35 \%$ & $-9.95 \%$ & $-7.10 \%$ & $0.15 \%$ & $6.30 \%$ & $10.25 \%$ & $-6.20 \%$ & $-3.55 \%$ & $-0.90 \%$ & $-7.55 \%$ & $-20.40 \%$ \\
\hline 78 GBR & $0.16 \%$ & $-1.06 \%$ & $-1.68 \%$ & $-5.06 \%$ & $-9.84 \%$ & $-8.49 \%$ & $-6.78 \%$ & $-1.96 \%$ & $2.08 \%$ & $4.56 \%$ & $-5.55 \%$ & $-3.39 \%$ & $-1.23 \%$ & $-7.39 \%$ & $-19.10 \%$ \\
\hline $79 \mathrm{CHE}$ & $-0.48 \%$ & $-2.13 \%$ & $-2.95 \%$ & $-6.13 \%$ & $-10.48 \%$ & $-6.58 \%$ & $-6.35 \%$ & $-4.73 \%$ & $-3.45 \%$ & $-2.88 \%$ & $-4.70 \%$ & $-3.18 \%$ & $-1.65 \%$ & $-7.18 \%$ & $-17.40 \%$ \\
\hline 80 NOR & $0.84 \%$ & $0.06 \%$ & $-0.32 \%$ & $-3.94 \%$ & $-9.16 \%$ & $-10.51 \%$ & $-7.23 \%$ & $0.96 \%$ & $7.93 \%$ & $12.44 \%$ & $-6.45 \%$ & $-3.61 \%$ & $-0.78 \%$ & $-7.61 \%$ & $-20.90 \%$ \\
\hline 81 XEF & $-0.48 \%$ & $-2.13 \%$ & $-2.95 \%$ & $-6.13 \%$ & $-10.48 \%$ & $-6.58 \%$ & $-6.35 \%$ & $-4.73 \%$ & $-3.45 \%$ & $-2.88 \%$ & $-4.70 \%$ & $-3.18 \%$ & $-1.65 \%$ & $-7.18 \%$ & $-17.40 \%$ \\
\hline 82 ALB & $-0.89 \%$ & $-2.81 \%$ & $-3.78 \%$ & $-6.81 \%$ & $-10.89 \%$ & $-5.34 \%$ & $-6.08 \%$ & $-6.51 \%$ & $-7.03 \%$ & $-7.69 \%$ & $-4.15 \%$ & $-3.04 \%$ & $-1.93 \%$ & $-7.04 \%$ & $-16.30 \%$ \\
\hline 83 BGR & $-0.81 \%$ & $-2.69 \%$ & $-3.63 \%$ & $-6.69 \%$ & $-10.81 \%$ & $-5.56 \%$ & $-6.13 \%$ & $6.19 \%$ & $-6.38 \%$ & $-6.81 \%$ & $-4.25 \%$ & $-3.06 \%$ & $-1.88 \%$ & $-7.06 \%$ & $-16.50 \%$ \\
\hline 84 BLR & $0.01 \%$ & $-1.31 \%$ & $-1.98 \%$ & $-5.31 \%$ & $-9.99 \%$ & $-8.04 \%$ & $-6.68 \%$ & $-2.61 \%$ & $0.77 \%$ & $2.81 \%$ & $-5.35 \%$ & $-3.34 \%$ & $-1.33 \%$ & $-7.34 \%$ & $-18.70 \%$ \\
\hline $85 \mathrm{HRV}$ & $-0.66 \%$ & $-2.44 \%$ & $-3.33 \%$ & $-6.44 \%$ & $-10.66 \%$ & $-6.01 \%$ & $-6.23 \%$ & $-5.54 \%$ & $-5.08 \%$ & $-5.06 \%$ & $-4.45 \%$ & $-3.11 \%$ & $-1.78 \%$ & $-7.11 \%$ & $-16.90 \%$ \\
\hline $86 \mid$ ROU & $-0.55 \%$ & $-2.25 \%$ & $-3.10 \%$ & $-6.25 \%$ & $-10.55 \%$ & $-6.35 \%$ & $-6.30 \%$ & $-5.05 \%$ & $-4.10 \%$ & $-3.75 \%$ & $-4.60 \%$ & $-3.15 \%$ & $-1.70 \%$ & $-7.15 \%$ & $-17.20 \%$ \\
\hline 87 RUS & $0.61 \%$ & $-0.31 \%$ & $-0.78 \%$ & $-4.31 \%$ & $-9.39 \%$ & $-9.84 \%$ & $-7.08 \%$ & $-0.01 \%$ & $5.98 \%$ & $9.81 \%$ & $-6.15 \%$ & $-3.54 \%$ & $-0.93 \%$ & $-7.54 \%$ & $-20.30 \%$ \\
\hline 88 UKR & $-0.40 \%$ & $-2.00 \%$ & $-2.80 \%$ & $-6.00 \%$ & $-10.40 \%$ & $-6.80 \%$ & $-6.40 \%$ & $-4.40 \%$ & $-2.80 \%$ & $-2.00 \%$ & $-4.80 \%$ & $-3.20 \%$ & $-1.60 \%$ & $-7.20 \%$ & $-17.60 \%$ \\
\hline 89 XEE & $-0.40 \%$ & $-2.00 \%$ & $-2.80 \%$ & $-6.00 \%$ & $-10.40 \%$ & $-6.80 \%$ & $-6.40 \%$ & $-4.40 \%$ & $-2.80 \%$ & $-2.00 \%$ & $-4.80 \%$ & $-3.20 \%$ & $-1.60 \%$ & $-7.20 \%$ & $-17.60 \%$ \\
\hline 90 XER & $-0.18 \%$ & $-1.63 \%$ & $-2.35 \%$ & $-5.63 \%$ & $-10.18 \%$ & $-7.48 \%$ & $-6.55 \%$ & $-3.43 \%$ & $-0.85 \%$ & $0.63 \%$ & $-5.10 \%$ & $-3.28 \%$ & $-1.45 \%$ & $-7.28 \%$ & $-18.20 \%$ \\
\hline $91 \mid$ KAZ & $-0.40 \%$ & $-2.00 \%$ & $-2.80 \%$ & $-6.00 \%$ & $-10.40 \%$ & $-6.80 \%$ & $-6.40 \%$ & $-4.40 \%$ & $-2.80 \%$ & $-2.00 \%$ & $-4.80 \%$ & $-3.20 \%$ & $-1.60 \%$ & $-7.20 \%$ & $-17.60 \%$ \\
\hline $92 \mid$ KGZ & $-0.96 \%$ & $-2.94 \%$ & $-3.93 \%$ & $-6.94 \%$ & $-10.96 \%$ & $-5.11 \%$ & $-6.03 \%$ & $-6.84 \%$ & $-7.68 \%$ & $-8.56 \%$ & $-4.05 \%$ & $-3.01 \%$ & $-1.98 \%$ & $-7.01 \%$ & $-16.10 \%$ \\
\hline 93 XSU & $-0.40 \%$ & $-2.00 \%$ & $-2.80 \%$ & $-6.00 \%$ & $-10.40 \%$ & $-6.80 \%$ & $-6.40 \%$ & $-4.40 \%$ & $-2.80 \%$ & $-2.00 \%$ & $-4.80 \%$ & $-3.20 \%$ & $-1.60 \%$ & $-7.20 \%$ & $-17.60 \%$ \\
\hline 94 ARM & $-0.96 \%$ & $-2.94 \%$ & $-3.93 \%$ & $-6.94 \%$ & $-10.96 \%$ & $-5.11 \%$ & $-6.03 \%$ & $-6.84 \%$ & $-7.68 \%$ & $-8.56 \%$ & $-4.05 \%$ & $-3.01 \%$ & $-1.98 \%$ & $-7.01 \%$ & $-16.10 \%$ \\
\hline 95 AZE & $-1.00 \%$ & $-3.00 \%$ & $-4.00 \%$ & $-7.00 \%$ & $-11.00 \%$ & $-5.00 \%$ & $-6.00 \%$ & $-7.00 \%$ & $-8.00 \%$ & $-9.00 \%$ & $-4.00 \%$ & $-3.00 \%$ & $-2.00 \%$ & $-7.00 \%$ & $-16.00 \%$ \\
\hline 96 GEO & $-0.81 \%$ & $-2.69 \%$ & $-3.63 \%$ & $-6.69 \%$ & $-10.81 \%$ & $-5.56 \%$ & $-6.13 \%$ & $-6.19 \%$ & $-6.38 \%$ & $-6.81 \%$ & $-4.25 \%$ & $-3.06 \%$ & $-1.88 \%$ & $-7.06 \%$ & $-16.50 \%$ \\
\hline $97 \mathrm{BHR}$ & $-2.05 \%$ & $-4.75 \%$ & $-6.10 \%$ & $-8.75 \%$ & $-12.05 \%$ & $-1.85 \%$ & $-5.30 \%$ & $-11.55 \%$ & $-17.10 \%$ & $-21.25 \%$ & $-2.60 \%$ & $-2.65 \%$ & $-2.70 \%$ & $-6.65 \%$ & $-13.20 \%$ \\
\hline $98 \mid \mathrm{IRN}$ & $-1.56 \%$ & $-3.94 \%$ & $-5.13 \%$ & $-7.94 \%$ & $-11.56 \%$ & $-3.31 \%$ & $-5.63 \%$ & $-9.44 \%$ & $-12.88 \%$ & $-15.56 \%$ & $-3.25 \%$ & $-2.81 \%$ & $-2.38 \%$ & $-6.81 \%$ & $-14.50 \%$ \\
\hline 99 |SR & $-1.68 \%$ & $-4.13 \%$ & $-5.35 \%$ & $-8.13 \%$ & $-11.68 \%$ & $-2.98 \%$ & $-5.55 \%$ & $-9.93 \%$ & $-13.85 \%$ & $-16.88 \%$ & $-3.10 \%$ & $-2.78 \%$ & $-2.45 \%$ & $-6.78 \%$ & $-14.20 \%$ \\
\hline 100JOR & $-1.68 \%$ & $-4.13 \%$ & $-5.35 \%$ & $-8.13 \%$ & $-11.68 \%$ & $-2.98 \%$ & $-5.55 \%$ & $-9.93 \%$ & $-13.85 \%$ & $-16.88 \%$ & $-3.10 \%$ & $-2.78 \%$ & $-2.45 \%$ & $-6.78 \%$ & $-14.20 \%$ \\
\hline $101 \mid \mathrm{KWT}$ & $-1.83 \%$ & $-4.38 \%$ & $-5.65 \%$ & $-8.38 \%$ & $-11.83 \%$ & $-2.53 \%$ & $-5.45 \%$ & $-10.58 \%$ & $-15.15 \%$ & $-18.63 \%$ & $-2.90 \%$ & $-2.73 \%$ & $-2.55 \%$ & $-6.73 \%$ & $-13.80 \%$ \\
\hline $102 \mathrm{OMN}$ & $-2.39 \%$ & $-5.31 \%$ & $-6.78 \%$ & $-9.31 \%$ & $-12.39 \%$ & $-0.84 \%$ & $-5.08 \%$ & $-13.01 \%$ & $-20.03 \%$ & $-25.19 \%$ & $-2.15 \%$ & $-2.54 \%$ & $-2.93 \%$ & $-6.54 \%$ & $-12.30 \%$ \\
\hline 103 QAT & $-2.13 \%$ & $-4.88 \%$ & $-6.25 \%$ & $-8.88 \%$ & $-12.13 \%$ & $-1.63 \%$ & $-5.25 \%$ & $11.88 \%$ & $-17.75 \%$ & $-22.13 \%$ & $-2.50 \%$ & $-2.63 \%$ & $-2.75 \%$ & $-6.63 \%$ & $-13.00 \%$ \\
\hline 104 SAU & $-2.24 \%$ & $-5.06 \%$ & $-6.48 \%$ & $-9.06 \%$ & $-12.24 \%$ & $-1.29 \%$ & $-5.18 \%$ & $-12.36 \%$ & $-18.73 \%$ & $-23.44 \%$ & $-2.35 \%$ & $-2.59 \%$ & $-2.83 \%$ & $-6.59 \%$ & $-12.70 \%$ \\
\hline 106 TUR & $-1.08 \%$ & $-3.13 \%$ & $-4.15 \%$ & $-7.13 \%$ & $-11.08 \%$ & $-4.78 \%$ & $-5.95 \%$ & $-7.33 \%$ & $-8.65 \%$ & $-9.88 \%$ & $-3.90 \%$ & $-2.98 \%$ & $-2.05 \%$ & $-6.98 \%$ & $-15.80 \%$ \\
\hline 105 ARE & $-2.16 \%$ & $-4.94 \%$ & $-6.33 \%$ & $-8.94 \%$ & $-12.16 \%$ & $-1.51 \%$ & $-5.23 \%$ & $-12.04 \%$ & $-18.08 \%$ & $-22.56 \%$ & $-2.45 \%$ & $-2.61 \%$ & $-2.78 \%$ & $-6.61 \%$ & $-12.90 \%$ \\
\hline 107XWS & $-1.68 \%$ & $-4.13 \%$ & $-5.35 \%$ & $-8.13 \%$ & $-11.68 \%$ & $-2.98 \%$ & $-5.55 \%$ & $-9.93 \%$ & $-13.85 \%$ & $-16.88 \%$ & $-3.10 \%$ & $-2.78 \%$ & $-2.45 \%$ & $-6.78 \%$ & $-14.20 \%$ \\
\hline 108 EGY & $-1.98 \%$ & $-4.63 \%$ & $-5.95 \%$ & $-8.63 \%$ & $-11.98 \%$ & $-2.08 \%$ & $-5.35 \%$ & $-11.23 \%$ & $-16.45 \%$ & $-20.38 \%$ & $-2.70 \%$ & $-2.68 \%$ & $-2.65 \%$ & $-6.68 \%$ & $-13.40 \%$ \\
\hline 109MAR & $-1.64 \%$ & $-4.06 \%$ & $-5.28 \%$ & $-8.06 \%$ & $-11.64 \%$ & $-3.09 \%$ & $-5.58 \%$ & $-9.76 \%$ & $-13.53 \%$ & $-16.44 \%$ & $-3.15 \%$ & $-2.79 \%$ & $-2.43 \%$ & $-6.79 \%$ & $-14.30 \%$ \\
\hline 110TUN & $-1.49 \%$ & $-3.81 \%$ & $-4.98 \%$ & $-7.81 \%$ & $-11.49 \%$ & $-3.54 \%$ & $-5.68 \%$ & $-9.11 \%$ & $-12.23 \%$ & $-14.69 \%$ & $-3.35 \%$ & $-2.84 \%$ & $-2.33 \%$ & $-6.84 \%$ & $-14.70 \%$ \\
\hline 111 XNF & $-1.49 \%$ & $-3.81 \%$ & $-4.98 \%$ & $-7.81 \%$ & $-11.49 \%$ & $-3.54 \%$ & $-5.68 \%$ & $-9.11 \%$ & $-12.23 \%$ & $-14.69 \%$ & $-3.35 \%$ & $-2.84 \%$ & $-2.33 \%$ & $-6.84 \%$ & $-14.70 \%$ \\
\hline 112 BEN & $-3.29 \%$ & $-6.81 \%$ & $-8.58 \%$ & $-10.81 \%$ & $-13.29 \%$ & $1.86 \%$ & $-4.48 \%$ & $-16.91 \%$ & $-27.83 \%$ & $-35.69 \%$ & $-0.95 \%$ & $-2.24 \%$ & $-3.53 \%$ & $-6.24 \%$ & $-9.90 \%$ \\
\hline $113 \mathrm{BFA}$ & $-3.10 \%$ & $-6.50 \%$ & $-8.20 \%$ & $-10.50 \%$ & $-13.10 \%$ & $1.30 \%$ & $-4.60 \%$ & $-16.10 \%$ & $-26.20 \%$ & $-33.50 \%$ & $-1.20 \%$ & $-2.30 \%$ & $-3.40 \%$ & $-6.30 \%$ & $-10.40 \%$ \\
\hline 114 CMR & $-3.44 \%$ & $-7.06 \%$ & $-8.88 \%$ & $-11.06 \%$ & $-13.44 \%$ & $2.31 \%$ & $-4.38 \%$ & $-17.56 \%$ & $-29.13 \%$ & $-37.44 \%$ & $-0.75 \%$ & $-2.19 \%$ & $-3.63 \%$ & $-6.19 \%$ & $-9.50 \%$ \\
\hline 115 CIV & $-3.44 \%$ & $-7.06 \%$ & $-8.88 \%$ & $-11.06 \%$ & $-13.44 \%$ & $2.31 \%$ & $-4.38 \%$ & $-17.56 \%$ & $-29.13 \%$ & $-37.44 \%$ & $-0.75 \%$ & $-2.19 \%$ & $-3.63 \%$ & $-6.19 \%$ & $-9.50 \%$ \\
\hline $116 \mathrm{GHA}$ & $-3.40 \%$ & $-7.00 \%$ & $-8.80 \%$ & $-11.00 \%$ & $-13.40 \%$ & $2.20 \%$ & $-4.40 \%$ & $-17.40 \%$ & $-28.80 \%$ & $-37.00 \%$ & $-0.80 \%$ & $-2.20 \%$ & $-3.60 \%$ & $-6.20 \%$ & $-9.60 \%$ \\
\hline $117 \mathrm{GIN}$ & $-3.25 \%$ & $-6.75 \%$ & $-8.50 \%$ & $-10.75 \%$ & $-13.25 \%$ & $1.75 \%$ & $-4.50 \%$ & $-16.75 \%$ & $-27.50 \%$ & $-35.25 \%$ & $-1.00 \%$ & $-2.25 \%$ & $-3.50 \%$ & $-6.25 \%$ & $-10.00 \%$ \\
\hline $118 \mathrm{NGA}$ & $-3.33 \%$ & $-6.88 \%$ & $-8.65 \%$ & $-10.88 \%$ & $-13.33 \%$ & $1.98 \%$ & $-4.45 \%$ & $-17.08 \%$ & $-28.15 \%$ & $-36.13 \%$ & $-0.90 \%$ & $-2.23 \%$ & $-3.55 \%$ & $-6.23 \%$ & $-9.80 \%$ \\
\hline 119 SEN & $-2.91 \%$ & $-6.19 \%$ & $-7.83 \%$ & $-10.19 \%$ & $-12.91 \%$ & $0.74 \%$ & $-4.73 \%$ & $-15.29 \%$ & $-24.58 \%$ & $-31.31 \%$ & $-1.45 \%$ & $-2.36 \%$ & $-3.28 \%$ & $-6.36 \%$ & $-10.90 \%$ \\
\hline 120 TGO & $-3.36 \%$ & $-6.94 \%$ & $-8.73 \%$ & $-10.94 \%$ & $-13.36 \%$ & $2.09 \%$ & $-4.43 \%$ & $-17.24 \%$ & $-28.48 \%$ & $-36.56 \%$ & $-0.85 \%$ & $-2.21 \%$ & $-3.58 \%$ & $-6.21 \%$ & $-9.70 \%$ \\
\hline 121 XWF & $-3.36 \%$ & $-6.94 \%$ & $-8.73 \%$ & $-10.94 \%$ & $-13.36 \%$ & $2.09 \%$ & $-4.43 \%$ & $-17.24 \%$ & $-28.48 \%$ & $-36.56 \%$ & $-0.85 \%$ & $-2.21 \%$ & $-3.58 \%$ & $-6.21 \%$ & $-9.70 \%$ \\
\hline 122 XCF & $-3.51 \%$ & $-7.19 \%$ & $-9.03 \%$ & $-11.19 \%$ & $-13.51 \%$ & $2.54 \%$ & $-4.33 \%$ & $-17.89 \%$ & $-29.78 \%$ & $-38.31 \%$ & $-0.65 \%$ & $-2.16 \%$ & $-3.68 \%$ & $-6.16 \%$ & $-9.30 \%$ \\
\hline $123 \times \mathrm{XAC}$ & $-4.00 \%$ & $-8.00 \%$ & $-10.00 \%$ & $-12.00 \%$ & $-14.00 \%$ & $4.00 \%$ & $-4.00 \%$ & $-20.00 \%$ & $-34.00 \%$ & $-44.00 \%$ & $0.00 \%$ & $-2.00 \%$ & $-4.00 \%$ & $-6.00 \%$ & $-8.00 \%$ \\
\hline 124 ETH & $-3.33 \%$ & $-6.88 \%$ & $-8.65 \%$ & $-10.88 \%$ & $-13.33 \%$ & $1.98 \%$ & $-4.45 \%$ & $-17.08 \%$ & $-28.15 \%$ & $-36.13 \%$ & $-0.90 \%$ & $-2.23 \%$ & $-3.55 \%$ & $-6.23 \%$ & $-9.80 \%$ \\
\hline 125 KEN & $-4.00 \%$ & $-8.00 \%$ & $-10.00 \%$ & $-12.00 \%$ & $-14.00 \%$ & $4.00 \%$ & $-4.00 \%$ & $-20.00 \%$ & $-34.00 \%$ & $-44.00 \%$ & $0.00 \%$ & $-2.00 \%$ & $-4.00 \%$ & $-6.00 \%$ & $-8.00 \%$ \\
\hline 126|MDG & $-2.58 \%$ & $-5.63 \%$ & $-7.15 \%$ & $-9.63 \%$ & $-12.58 \%$ & $-0.28 \%$ & $-4.95 \%$ & $-13.83 \%$ & $-21.65 \%$ & $-27.38 \%$ & $-1.90 \%$ & $-2.48 \%$ & $-3.05 \%$ & $-6.48 \%$ & $-11.80 \%$ \\
\hline $127 \mathrm{MWI}$ & $-3.03 \%$ & $-6.38 \%$ & $-8.05 \%$ & $-10.38 \%$ & $-13.03 \%$ & $1.08 \%$ & $-4.65 \%$ & $-15.78 \%$ & $-25.55 \%$ & $-32.63 \%$ & $-1.30 \%$ & $-2.33 \%$ & $-3.35 \%$ & $-6.33 \%$ & $-10.60 \%$ \\
\hline 128 MUS & $-2.43 \%$ & $-5.38 \%$ & $-6.85 \%$ & $-9.38 \%$ & $-12.43 \%$ & $-0.73 \%$ & $-5.05 \%$ & $-13.18 \%$ & $-20.35 \%$ & $-25.63 \%$ & $-2.10 \%$ & $-2.53 \%$ & $-2.95 \%$ & $-6.53 \%$ & $-12.20 \%$ \\
\hline $129 \mid \mathrm{MOZ}$ & $-2.61 \%$ & $-5.69 \%$ & $-7.23 \%$ & $-9.69 \%$ & $-12.61 \%$ & $-0.16 \%$ & $-4.93 \%$ & $-13.99 \%$ & $-21.98 \%$ & $-27.81 \%$ & $-1.85 \%$ & $-2.46 \%$ & $-3.08 \%$ & $-6.46 \%$ & $-11.70 \%$ \\
\hline 130 RWA & $-3.85 \%$ & $-7.75 \%$ & $-9.70 \%$ & $-11.75 \%$ & $-13.85 \%$ & $3.55 \%$ & $-4.10 \%$ & $-19.35 \%$ & $-32.70 \%$ & $-42.25 \%$ & $-0.20 \%$ & $-2.05 \%$ & $-3.90 \%$ & $-6.05 \%$ & $-8.40 \%$ \\
\hline 131 TZA & $-3.51 \%$ & $-7.19 \%$ & $-9.03 \%$ & $-11.19 \%$ & $-13.51 \%$ & $2.54 \%$ & $-4.33 \%$ & $-17.89 \%$ & $-29.78 \%$ & $-38.31 \%$ & $-0.65 \%$ & $-2.16 \%$ & $-3.68 \%$ & $-6.16 \%$ & $-9.30 \%$ \\
\hline 132 UGA & $-3.89 \%$ & $-7.81 \%$ & $-9.78 \%$ & $-11.81 \%$ & $-13.89 \%$ & $3.66 \%$ & $-4.08 \%$ & $-19.51 \%$ & $-33.03 \%$ & $-42.69 \%$ & $-0.15 \%$ & $-2.04 \%$ & $-3.93 \%$ & $-6.04 \%$ & $-8.30 \%$ \\
\hline $133 \mathrm{ZMB}$ & $-3.03 \%$ & $-6.38 \%$ & $-8.05 \%$ & $-10.38 \%$ & $-13.03 \%$ & $1.08 \%$ & $-4.65 \%$ & $-15.78 \%$ & $-25.55 \%$ & $-32.63 \%$ & $-1.30 \%$ & $-2.33 \%$ & $-3.35 \%$ & $-6.33 \%$ & $-10.60 \%$ \\
\hline 134 ZWE & $-2.61 \%$ & $-5.69 \%$ & $-7.23 \%$ & $-9.69 \%$ & $-12.61 \%$ & $-0.16 \%$ & $-4.93 \%$ & $-13.99 \%$ & $-21.98 \%$ & $-27.81 \%$ & $-1.85 \%$ & $-2.46 \%$ & $-3.08 \%$ & $-6.46 \%$ & $-11.70 \%$ \\
\hline 135 XEC & $-2.61 \%$ & $-5.69 \%$ & $-7.23 \%$ & $-9.69 \%$ & $-12.61 \%$ & $-0.16 \%$ & $-4.93 \%$ & $-13.99 \%$ & $-21.98 \%$ & $-27.81 \%$ & $-1.85 \%$ & $-2.46 \%$ & $-3.08 \%$ & $-6.46 \%$ & $-11.70 \%$ \\
\hline 136 BWA & $-2.31 \%$ & $-5.19 \%$ & $-6.63 \%$ & $-9.19 \%$ & $-12.31 \%$ & $-1.06 \%$ & $-5.13 \%$ & $-12.69 \%$ & $-19.38 \%$ & $-24.31 \%$ & $-2.25 \%$ & $-2.56 \%$ & $-2.88 \%$ & $-6.56 \%$ & $-12.50 \%$ \\
\hline 137NAM & $-2.28 \%$ & $-5.13 \%$ & $-6.55 \%$ & $-9.13 \%$ & $-12.28 \%$ & $-1.18 \%$ & $-5.15 \%$ & $-12.53 \%$ & $-19.05 \%$ & $-23.88 \%$ & $-2.30 \%$ & $-2.58 \%$ & $-2.85 \%$ & $-6.58 \%$ & $-12.60 \%$ \\
\hline 138 ZAF & $-1.86 \%$ & $-4.44 \%$ & $-5.73 \%$ & $-8.44 \%$ & $-11.86 \%$ & $-2.41 \%$ & $-5.43 \%$ & $-10.74 \%$ & $-15.48 \%$ & $-19.06 \%$ & $-2.85 \%$ & $-2.71 \%$ & $-2.58 \%$ & $-6.71 \%$ & $-13.70 \%$ \\
\hline 139 XSC & $-1.86 \%$ & $-4.44 \%$ & $-5.73 \%$ & $-8.44 \%$ & $-11.86 \%$ & $-2.41 \%$ & $-5.43 \%$ & $-10.74 \%$ & $-15.48 \%$ & $-19.06 \%$ & $-2.85 \%$ & $-2.71 \%$ & $-2.58 \%$ & $-6.71 \%$ & $-13.70 \%$ \\
\hline 140XTW & $-1.00 \%$ & $-3.00 \%$ & $-4.00 \%$ & $-7.00 \%$ & $-11.00 \%$ & $-5.00 \%$ & $-6.00 \%$ & $-7.00 \%$ & $-8.00 \%$ & $-9.00 \%$ & $-4.00 \%$ & $-3.00 \%$ & $-2.00 \%$ & $-7.00 \%$ & $-16.00 \%$ \\
\hline
\end{tabular}


Table A4-1. Agriculture: percentage variation in multi-factor productivity. Negative values in red.

\begin{tabular}{|c|c|c|c|c|c|c|c|c|c|c|c|c|c|c|}
\hline Code & Base $Y$ & ref.imp. & adj & Base T & Delta T & Base $P$ & Delta P & P/T Ratio & C/T Ratio & $+1^{\circ} \mathrm{C}$ & $+2^{\circ} \mathrm{C}$ & $+3^{\circ} \mathrm{C}$ & $+4^{\circ} \mathrm{C}$ & $+5^{\circ} \mathrm{C}$ \\
\hline 1 AUS & 5000 & -6.5 & $-0.19 \%$ & 21.95 & 2.7 & 1.59 & -0.02 & -0.0074 & 54.7037 & $-1.91 \%$ & $-4.01 \%$ & $-6.31 \%$ & $-8.81 \%$ & $-11.51 \%$ \\
\hline $2 \mathrm{NZL}$ & 500 & -3.8 & $-2.03 \%$ & 12.08 & 2 & 3.58 & 0.1 & 0.0500 & 73.8500 & $1.39 \%$ & $0.80 \%$ & $-1.78 \%$ & $-6.35 \%$ & $-12.90 \%$ \\
\hline $3 X O C$ & 4000 & -9.1 & $0.86 \%$ & 25.24 & 1.84 & 5.14 & 0.23 & 0.1250 & 80.2717 & $-3.06 \%$ & $-6.37 \%$ & $-9.92 \%$ & $-13.73 \%$ & $-17.78 \%$ \\
\hline $4 \mathrm{CHN}$ & 10000 & -5.1 & $-6.86 \%$ & 5.3 & 3.4 & 2.37 & 0.21 & 0.0618 & 43.4412 & $0.68 \%$ & $1.26 \%$ & $1.74 \%$ & $2.11 \%$ & $2.40 \%$ \\
\hline 5 HKG & 5000 & -7.5 & $-0.83 \%$ & 22.84 & 2.14 & 4.5 & 0.13 & 0.0607 & 69.0187 & $-2.02 \%$ & $-4.24 \%$ & $-6.65 \%$ & $-9.27 \%$ & $-12.08 \%$ \\
\hline 6 JPN & 500 & -5.5 & $-0.34 \%$ & 12.13 & 2.94 & 4.43 & 0.23 & 0.0782 & 50.2381 & $0.28 \%$ & $-1.43 \%$ & $-5.13 \%$ & $-10.81 \%$ & $-18.48 \%$ \\
\hline $7 \mathrm{KOR}$ & 500 & -5.1 & $-0.24 \%$ & 12.07 & 2.99 & 3.16 & 0.26 & 0.0870 & 49.3980 & $0.36 \%$ & $-1.26 \%$ & $-4.88 \%$ & $-10.48 \%$ & $-18.06 \%$ \\
\hline $8 \mathrm{MNG}$ & 10000 & -3.1 & $-6.52 \%$ & -0.4 & 3.66 & 1.01 & 0.13 & 0.0355 & 40.3552 & $1.24 \%$ & $2.38 \%$ & $3.41 \%$ & $4.35 \%$ & $5.19 \%$ \\
\hline 9TWN & 5000 & -7.1 & $-0.48 \%$ & 22.84 & 2.14 & 4.5 & 0.13 & 0.0607 & 69.0187 & $-2.02 \%$ & $-4.24 \%$ & $-6.65 \%$ & $-9.27 \%$ & $-12.08 \%$ \\
\hline 10XEA & 5000 & -9.1 & $-0.95 \%$ & 25.3 & 2.34 & 5.07 & 0.22 & 0.0940 & 63.1197 & $-2.53 \%$ & $-5.27 \%$ & $-8.20 \%$ & $-11.33 \%$ & $-14.66 \%$ \\
\hline 11 BRN & 4000 & -10.5 & $-0.30 \%$ & 25.41 & 2.17 & 7.9 & 0.43 & 0.1982 & 68.0645 & $-3.17 \%$ & $-6.58 \%$ & $-10.24 \%$ & $-14.16 \%$ & $-18.32 \%$ \\
\hline 12 KHM & 5000 & -9.1 & $-0.95 \%$ & 25.3 & 2.34 & 5.07 & 0.22 & 0.0940 & 63.1197 & $-2.53 \%$ & $-5.27 \%$ & $-8.20 \%$ & $-11.33 \%$ & $-14.66 \%$ \\
\hline 13 IDN & 4000 & -10.9 & $-0.55 \%$ & 25.53 & 2.2 & 0.13 & 0.39 & 0.1773 & 67.1364 & $-3.20 \%$ & $-6.65 \%$ & $-10.35 \%$ & $-14.30 \%$ & $-18.49 \%$ \\
\hline 14 LAO & 4000 & -8.1 & $-0.31 \%$ & 21.89 & 2.53 & 3.75 & 0.2 & 0.0791 & 58.3794 & $-2.35 \%$ & $-4.94 \%$ & $-7.78 \%$ & $-10.87 \%$ & $-14.21 \%$ \\
\hline 15 MYS & 4000 & -10.5 & $-0.29 \%$ & 25.4 & 2.21 & 7.86 & 0.48 & 0.2172 & 66.8326 & $-3.17 \%$ & $-6.59 \%$ & $-10.26 \%$ & $-14.17 \%$ & $-18.34 \%$ \\
\hline 16 $\mathrm{PHL}$ & 5000 & -9.1 & $-0.42 \%$ & 26.23 & 2.06 & 6.76 & 0.5 & 0.2427 & 71.6990 & $-2.68 \%$ & $-5.56 \%$ & $-8.64 \%$ & $-11.91 \%$ & $-15.39 \%$ \\
\hline 17 $S G P$ & 4000 & -11.2 & $0.21 \%$ & 26.96 & 2.11 & 7.5 & 0.18 & 0.0853 & 70.0000 & $-3.54 \%$ & $-7.33 \%$ & $-11.37 \%$ & $-15.66 \%$ & $-20.20 \%$ \\
\hline 18 THA & 4000 & -9.1 & $0.65 \%$ & 24.63 & 2.46 & 4.27 & 0.18 & 0.0732 & 60.0407 & $-3.02 \%$ & $-6.28 \%$ & $-9.80 \%$ & $-13.56 \%$ & $-17.57 \%$ \\
\hline 19VNM & 4000 & -8.6 & $-0.04 \%$ & 23.05 & 2.36 & 4.42 & 0.25 & 0.1059 & 62.5847 & $-2.61 \%$ & $-5.47 \%$ & $-8.58 \%$ & $-11.93 \%$ & $-15.54 \%$ \\
\hline 20XSE & 5000 & -9.1 & $-0.95 \%$ & 25.3 & 2.34 & 5.07 & 0.22 & 0.0940 & 63.1197 & $-2.53 \%$ & $-5.27 \%$ & $-8.20 \%$ & $-11.33 \%$ & $-14.66 \%$ \\
\hline 21 BGD & 5000 & -7.1 & $0.49 \%$ & 24.24 & 2.54 & 3.9 & 0.4 & 0.1575 & 58.1496 & $-2.34 \%$ & $-4.89 \%$ & $-7.63 \%$ & $-10.57 \%$ & $-13.71 \%$ \\
\hline 22 IND & 5000 & -7.4 & $-0.30 \%$ & 23.24 & 2.77 & 2.65 & 0.27 & 0.0975 & 53.3213 & $-2.17 \%$ & $-4.53 \%$ & $-7.10 \%$ & $-9.86 \%$ & $-12.82 \%$ \\
\hline 23 NPL & 500 & -6.4 & $-6.19 \%$ & 11.24 & 3.15 & 4.99 & 0.43 & 0.1365 & 46.8889 & $1.91 \%$ & $1.83 \%$ & $-0.24 \%$ & $-4.29 \%$ & $-10.34 \%$ \\
\hline 24) PAK & 4000 & -6.1 & $-0.35 \%$ & 18.82 & 3.38 & 0.87 & 0.05 & 0.0148 & 43.6982 & $-1.66 \%$ & $-3.57 \%$ & $-5.73 \%$ & $-8.14 \%$ & $-10.80 \%$ \\
\hline 25 LKA & 5000 & -9.9 & $-0.93 \%$ & 26.87 & 2.04 & 4.87 & 0.53 & 0.2598 & 72.4020 & $-2.80 \%$ & $-5.81 \%$ & $-9.01 \%$ & $-12.41 \%$ & $-16.01 \%$ \\
\hline 26)XSA & 5000 & -7.4 & $-0.30 \%$ & 23.24 & 2.77 & 2.65 & 0.27 & 0.0975 & 53.3213 & $-2.17 \%$ & $-4.53 \%$ & $-7.10 \%$ & $-9.86 \%$ & $-12.82 \%$ \\
\hline 27 CAN & 10000 & -0.4 & $-4.87 \%$ & -4.13 & 4.54 & 1.83 & 0.23 & 0.0507 & 32.5330 & $1.59 \%$ & $3.08 \%$ & $4.48 \%$ & $5.77 \%$ & $6.96 \%$ \\
\hline 28 USA & 10000 & -4.9 & $-5.62 \%$ & 8.52 & 3.63 & 2.37 & 0.13 & 0.0358 & 40.6887 & $0.35 \%$ & $0.60 \%$ & $0.76 \%$ & $0.81 \%$ & $0.77 \%$ \\
\hline 29 MEX & 3000 & -7.1 & $0.88 \%$ & 19.35 & 2.89 & 2.54 & -0.2 & -0.0692 & 51.1073 & $-2.34 \%$ & $-5.01 \%$ & $-8.02 \%$ & $-11.35 \%$ & $-15.02 \%$ \\
\hline 30 XNA & 10000 & -4.9 & $-5.62 \%$ & 8.52 & 3.63 & 2.37 & 0.13 & 0.0358 & 40.6887 & $0.35 \%$ & $0.60 \%$ & $0.76 \%$ & $0.81 \%$ & $0.77 \%$ \\
\hline 31 ARG & 2000 & -4.6 & $-0.21 \%$ & 14.57 & 2.21 & 2.1 & 0.02 & 0.0090 & 66.8326 & $-0.97 \%$ & $-2.43 \%$ & $-4.39 \%$ & $-6.84 \%$ & $-9.79 \%$ \\
\hline $32 \mathrm{BOL}$ & 3000 & -8.4 & $0.13 \%$ & 19.82 & 3.17 & 4.11 & -0.06 & -0.0189 & 46.5931 & $-2.53 \%$ & $-5.39 \%$ & $-8.58 \%$ & $-12.10 \%$ & $-15.95 \%$ \\
\hline 33 BRA & 5000 & -7.9 & $0.21 \%$ & 24.91 & 2.91 & 4.01 & -0.08 & -0.0275 & 50.7560 & $-2.51 \%$ & $-5.22 \%$ & $-8.13 \%$ & $-11.23 \%$ & $-14.54 \%$ \\
\hline $34 \mid \mathrm{CHL}$ & 10000 & -4.9 & $-5.24 \%$ & 10.61 & 2.27 & 2.87 & -0.19 & -0.0837 & 65.0661 & $0.20 \%$ & $0.29 \%$ & $0.29 \%$ & $0.19 \%$ & $-0.01 \%$ \\
\hline $35 \mathrm{COL}$ & 3500 & -10.6 & $0.11 \%$ & 23.95 & 2.76 & 6.37 & 0.14 & 0.0507 & 53.5145 & $-3.30 \%$ & $-6.88 \%$ & $-10.74 \%$ & $-14.89 \%$ & $-19.32 \%$ \\
\hline 36|ECU & 3000 & -11.1 & $-0.75 \%$ & 21.86 & 2.49 & 7.73 & 0.43 & 0.1727 & 59.3173 & $-3.11 \%$ & $-6.55 \%$ & $-10.32 \%$ & $-14.42 \%$ & $-18.86 \%$ \\
\hline 37|PRY & 5000 & -7.3 & $0.23 \%$ & 23.9 & 2.99 & 2.89 & 0 & 0.0000 & 49.3980 & $-2.32 \%$ & $-4.83 \%$ & $-7.54 \%$ & $-10.45 \%$ & $-13.56 \%$ \\
\hline 38 PER & 2500 & -9.8 & $0.51 \%$ & 19.89 & 2.91 & 5.6 & 0.22 & 0.0756 & 50.7560 & $-3.02 \%$ & $-6.45 \%$ & $-10.26 \%$ & $-14.48 \%$ & $-19.10 \%$ \\
\hline 39 URY & 3000 & -5.6 & $0.75 \%$ & 18.15 & 2.09 & 2.51 & 0.21 & 0.1005 & 70.6699 & $-1.80 \%$ & $-3.93 \%$ & $-6.39 \%$ & $-9.19 \%$ & $-12.31 \%$ \\
\hline 40 VEN & 4000 & -10.2 & $0.17 \%$ & 25.2 & 2.85 & 3.35 & -0.25 & -0.0877 & 51.8246 & $-3.21 \%$ & $-6.66 \%$ & $-10.36 \%$ & $-14.31 \%$ & $-18.51 \%$ \\
\hline 41 XSM & 3000 & -5.6 & $0.75 \%$ & 18.15 & 2.09 & 2.51 & 0.21 & 0.1005 & 70.6699 & $-1.80 \%$ & $-3.93 \%$ & $-6.39 \%$ & $-9.19 \%$ & $-12.31 \%$ \\
\hline $42 \mathrm{CRI}$ & 4500 & -9.7 & $-0.15 \%$ & 25.96 & 2.26 & 3.44 & -0.16 & -0.0708 & 65.3540 & $-2.95 \%$ & $-6.12 \%$ & $-9.52 \%$ & $-13.13 \%$ & $-16.97 \%$ \\
\hline 43 GTM & 4500 & -8.5 & $-0.05 \%$ & 24.15 & 2.75 & 2.79 & -0.24 & -0.0873 & 53.7091 & $-2.61 \%$ & $-5.44 \%$ & $-8.49 \%$ & $-11.76 \%$ & $-15.25 \%$ \\
\hline 44 HND & 4500 & -8.8 & $-0.16 \%$ & 24.52 & 2.45 & 2.49 & -0.25 & -0.1020 & 60.2857 & $-2.66 \%$ & $-5.54 \%$ & $-8.64 \%$ & $-11.96 \%$ & $-15.50 \%$ \\
\hline 45 NIC & 4500 & -9.1 & $0.20 \%$ & 25.5 & 2.38 & 2.34 & -0.22 & -0.0924 & 62.0588 & $-2.87 \%$ & $-5.95 \%$ & $-9.26 \%$ & $-12.79 \%$ & $-16.54 \%$ \\
\hline $46 \mid S L V$ & 4500 & -9.8 & $-0.40 \%$ & 25.89 & 2.2 & 5.35 & 0.14 & 0.0636 & 67.1364 & $-2.93 \%$ & $-6.07 \%$ & $-9.44 \%$ & $-13.03 \%$ & $-16.84 \%$ \\
\hline 47PAN & 4500 & -9.0 & $0.39 \%$ & 25.54 & 2.6 & 2.06 & -0.22 & -0.0846 & 56.8077 & $-2.90 \%$ & $-6.02 \%$ & $-9.36 \%$ & $-12.93 \%$ & $-16.71 \%$ \\
\hline 48 XCA & 4500 & -9.1 & $0.20 \%$ & 25.5 & 2.38 & 2.34 & -0.22 & -0.0924 & 62.0588 & $-2.87 \%$ & $-5.95 \%$ & $-9.26 \%$ & $-12.79 \%$ & $-16.54 \%$ \\
\hline 49 DOM & 5000 & -8.0 & $0.13 \%$ & 25.28 & 2.19 & 2.22 & -0.25 & -0.1142 & 67.4429 & $-2.51 \%$ & $-5.22 \%$ & $-8.13 \%$ & $-11.24 \%$ & $-14.55 \%$ \\
\hline 50/JAM & 5000 & -8.2 & $0.52 \%$ & 26.34 & 2.04 & 2.27 & -0.23 & -0.1127 & 72.4020 & $-2.70 \%$ & $-5.60 \%$ & $-8.70 \%$ & $-12.00 \%$ & $-15.50 \%$ \\
\hline 51|PRI & 5000 & -8.2 & $-0.10 \%$ & 25.38 & 1.95 & 1.82 & -0.26 & -0.1333 & 75.7436 & $-2.50 \%$ & $-5.19 \%$ & $-8.09 \%$ & $-11.18 \%$ & $-14.47 \%$ \\
\hline 52 TTO & 4500 & -9.4 & $-0.11 \%$ & 25.8 & 2.01 & 2.26 & -0.3 & -0.1493 & 73.4826 & $-2.88 \%$ & $-5.98 \%$ & $-9.30 \%$ & $-12.84 \%$ & $-16.60 \%$ \\
\hline 53 XCB & 5000 & -8.2 & $0.52 \%$ & 26.34 & 2.04 & 2.27 & -0.23 & -0.1127 & 72.4020 & $-2.70 \%$ & $-5.60 \%$ & $-8.70 \%$ & $-12.00 \%$ & $-15.50 \%$ \\
\hline 54|AUT & 10000 & -3.0 & $-4.01 \%$ & 7.89 & 3.15 & 2.62 & 0 & 0.0000 & 46.8889 & $0.43 \%$ & $0.76 \%$ & $0.98 \%$ & $1.11 \%$ & $1.14 \%$ \\
\hline $55 \mathrm{BEL}$ & 10000 & -2.5 & $-2.85 \%$ & 10.18 & 2.66 & 2.62 & 0.03 & 0.0113 & 55.5263 & $0.22 \%$ & $0.34 \%$ & $0.36 \%$ & $0.28 \%$ & $0.10 \%$ \\
\hline 56 CYP & 4000 & -5.2 & $0.60 \%$ & 19.18 & 2.63 & 1.38 & -0.16 & -0.0608 & 56.1597 & $-1.69 \%$ & $-3.62 \%$ & $-5.80 \%$ & $-8.24 \%$ & $-10.91 \%$ \\
\hline 57 CZE & 10000 & -2.6 & $-3.48 \%$ & 8.2 & 3.11 & 2.34 & 0.03 & 0.0096 & 47.4920 & $0.40 \%$ & $0.70 \%$ & $0.90 \%$ & $1.00 \%$ & $1.00 \%$ \\
\hline 58 DNK & 10000 & -1.4 & $-2.42 \%$ & 8.11 & 2.66 & 2.38 & 0.14 & 0.0526 & 55.5263 & $0.42 \%$ & $0.75 \%$ & $0.98 \%$ & $1.10 \%$ & $1.13 \%$ \\
\hline 59 EST & 10000 & -1.0 & $-2.92 \%$ & 4.66 & 3.64 & 1.96 & 0.17 & 0.0467 & 40.5769 & $0.74 \%$ & $1.37 \%$ & $1.91 \%$ & $2.34 \%$ & $2.68 \%$ \\
\hline $60 \mathrm{FIN}$ & 10000 & 0.0 & $-3.22 \%$ & 0.01 & 4.14 & 1.9 & 0.23 & 0.0556 & 35.6763 & $1.19 \%$ & $2.27 \%$ & $3.26 \%$ & $4.15 \%$ & $4.94 \%$ \\
\hline 61 FRA & 10000 & -3.2 & $-3.21 \%$ & 11.26 & 2.85 & 2.75 & -0.12 & -0.0421 & 51.8246 & $0.10 \%$ & $0.11 \%$ & $0.01 \%$ & $-0.18 \%$ & $-0.48 \%$ \\
\hline 62 DEU & 10000 & -2.4 & $-3.17 \%$ & 8.76 & 2.85 & 2.54 & 0.05 & 0.0175 & 51.8246 & $0.35 \%$ & $0.60 \%$ & $0.76 \%$ & $0.81 \%$ & $0.77 \%$ \\
\hline 63 GRC & 3000 & -4.6 & $-0.26 \%$ & 15.59 & 3.05 & 1.63 & -0.2 & -0.0656 & 48.4262 & $-1.11 \%$ & $-2.56 \%$ & $-4.34 \%$ & $-6.45 \%$ & $-8.89 \%$ \\
\hline 64 HUN & 10000 & -3.0 & $-3.27 \%$ & 10.28 & 3.36 & 2.03 & -0.03 & -0.0089 & 43.9583 & $0.18 \%$ & $0.27 \%$ & $0.25 \%$ & $0.14 \%$ & $-0.07 \%$ \\
\hline $65 \mid \mathrm{IRL}$ & 10000 & -2.1 & $-2.63 \%$ & 10.02 & 1.84 & 3.29 & 0.09 & 0.0489 & 80.2717 & $0.29 \%$ & $0.48 \%$ & $0.57 \%$ & $0.56 \%$ & $0.45 \%$ \\
\hline 66 ITA & 1500 & -4.1 & $0.23 \%$ & 13.4 & 3.02 & 2.05 & -0.13 & -0.0430 & 48.9073 & $-0.77 \%$ & $-2.21 \%$ & $-4.30 \%$ & $-7.06 \%$ & $-10.48 \%$ \\
\hline 67)LVA & 10000 & -1.4 & $-3.06 \%$ & 5.67 & 3.48 & 2.04 & 0.16 & 0.0460 & 42.4425 & $0.64 \%$ & $1.18 \%$ & $1.62 \%$ & $1.96 \%$ & $2.20 \%$ \\
\hline 68 LTU & 10000 & -1.7 & $-3.06 \%$ & 6.6 & 3.36 & 2.05 & 0.13 & 0.0387 & 43.9583 & $0.55 \%$ & $1.00 \%$ & $1.35 \%$ & $1.60 \%$ & $1.75 \%$ \\
\hline $69 \mid$ LUX & 10000 & -2.7 & $-3.13 \%$ & 9.76 & 2.82 & 2.93 & 0.01 & 0.0035 & 52.3759 & $0.25 \%$ & $0.41 \%$ & $0.46 \%$ & $0.42 \%$ & $0.27 \%$ \\
\hline 70|MLT & 4000 & -5.1 & $0.37 \%$ & 18.85 & 2.44 & 0.86 & -0.1 & -0.0410 & 60.5328 & $-1.58 \%$ & $-3.41 \%$ & $-5.49 \%$ & $-7.81 \%$ & $-10.39 \%$ \\
\hline
\end{tabular}


Table A4-2. Agriculture: percentage variation in multi-factor productivity. Negative values in red.

\begin{tabular}{|c|c|c|c|c|c|c|c|c|c|c|c|c|c|c|}
\hline Code & Base $Y$ & ref.imp. & adj & Base T & Delta T & Base $\mathbf{P}$ & Delta P & P/T Ratio & C/T Ratio & $+1^{\circ} \mathrm{C}$ & $+2^{\circ} \mathrm{C}$ & $+3^{\circ} \mathrm{C}$ & $+4^{\circ} \mathrm{C}$ & $+5^{\circ} \mathrm{C}$ \\
\hline $71 \mid$ NLD & 10000 & -2.1 & $-2.57 \%$ & 10.03 & 2.52 & 2.49 & 0.08 & 0.0317 & 58.6111 & $0.24 \%$ & $0.38 \%$ & $0.42 \%$ & $0.37 \%$ & $0.21 \%$ \\
\hline $72 \mid \mathrm{POL}$ & 10000 & -2.2 & $-3.19 \%$ & 8.38 & 2.26 & 2.02 & 0.07 & 0.0310 & 65.3540 & $0.42 \%$ & $0.74 \%$ & $0.96 \%$ & $1.08 \%$ & $1.10 \%$ \\
\hline $73 \mid$ PRT & 2500 & -4.4 & $0.55 \%$ & 15.53 & 2.69 & 2.13 & -0.32 & -0.1190 & 54.9071 & $-1.26 \%$ & $-2.92 \%$ & $-4.97 \%$ & $-7.42 \%$ & $-10.27 \%$ \\
\hline 74 SVK & 10000 & -2.8 & $-3.44 \%$ & 8.88 & 3.28 & 2.23 & -0.01 & -0.0030 & 45.0305 & $0.33 \%$ & $0.55 \%$ & $0.68 \%$ & $0.70 \%$ & $0.63 \%$ \\
\hline 75 SVN & 10000 & -3.3 & $-3.74 \%$ & 9.61 & 3.27 & 2.43 & -0.07 & -0.0214 & 45.1682 & $0.25 \%$ & $0.41 \%$ & $0.46 \%$ & $0.42 \%$ & $0.27 \%$ \\
\hline $76 \mid$ ESP & 2000 & -4.3 & $-0.25 \%$ & 14 & 2.95 & 1.8 & -0.25 & -0.0847 & 50.0678 & $-0.87 \%$ & $-2.23 \%$ & $-4.09 \%$ & $-6.44 \%$ & $-9.29 \%$ \\
\hline 77|SWE & 10000 & -0.5 & $-3.27 \%$ & 1.75 & 3.49 & 2.2 & 0.22 & 0.0630 & 42.3209 & $1.03 \%$ & $1.96 \%$ & $2.79 \%$ & $3.52 \%$ & $4.15 \%$ \\
\hline 78 GBR & 10000 & -1.6 & $-2.35 \%$ & 9.22 & 2.16 & 2.81 & 0.09 & 0.0417 & 68.3796 & $0.34 \%$ & $0.58 \%$ & $0.73 \%$ & $0.77 \%$ & $0.72 \%$ \\
\hline $79 \mathrm{CHE}$ & 10000 & -3.1 & $-4.30 \%$ & 7.22 & 3.07 & 3.41 & -0.05 & -0.0163 & 48.1107 & $0.50 \%$ & $0.89 \%$ & $1.19 \%$ & $1.39 \%$ & $1.49 \%$ \\
\hline 80 NOR & 10000 & 0.0 & $-3.10 \%$ & 0.89 & 3.3 & 2.89 & 0.31 & 0.0939 & 44.7576 & $1.12 \%$ & $2.14 \%$ & $3.06 \%$ & $3.88 \%$ & $4.60 \%$ \\
\hline $81 \mid \mathrm{XEF}$ & 10000 & -3.1 & $-4.30 \%$ & 7.22 & 3.07 & 3.41 & -0.05 & -0.0163 & 48.1107 & $0.50 \%$ & $0.89 \%$ & $1.19 \%$ & $1.39 \%$ & $1.49 \%$ \\
\hline $82 \mid A L B$ & 1500 & -4.1 & $0.16 \%$ & 13.3 & 3.21 & 2.28 & -0.22 & -0.0685 & 46.0125 & $-0.75 \%$ & $-2.16 \%$ & $-4.23 \%$ & $-6.97 \%$ & $-10.36 \%$ \\
\hline $83 \mid \mathrm{BGR}$ & 1000 & -3.9 & $-0.67 \%$ & 12.23 & 3.29 & 1.74 & -0.11 & -0.0334 & 44.8936 & $-0.08 \%$ & $-1.16 \%$ & $-3.22 \%$ & $-6.29 \%$ & $-10.34 \%$ \\
\hline $84 \mid B L R$ & 10000 & -2.0 & $-3.24 \%$ & 6.82 & 3.53 & 2.01 & 0.1 & 0.0283 & 41.8414 & $0.52 \%$ & $0.95 \%$ & $1.27 \%$ & $1.50 \%$ & $1.62 \%$ \\
\hline $85 \mathrm{HRV}$ & 500 & -3.5 & $-1.89 \%$ & 11.43 & 3.28 & 2.29 & -0.09 & -0.0274 & 45.0305 & $1.43 \%$ & $0.88 \%$ & $-1.66 \%$ & $-6.19 \%$ & $-12.70 \%$ \\
\hline $86 \mid$ ROU & 10000 & -3.3 & $-3.54 \%$ & 10.25 & 3.4 & 1.96 & -0.06 & -0.0176 & 43.4412 & $0.19 \%$ & $0.27 \%$ & $0.26 \%$ & $0.15 \%$ & $-0.07 \%$ \\
\hline $87 \mid R U S$ & 10000 & -0.6 & $-5.20 \%$ & -4.64 & 4.6 & 1.53 & 0.23 & 0.0500 & 32.1087 & $1.64 \%$ & $3.18 \%$ & $4.62 \%$ & $5.97 \%$ & $7.21 \%$ \\
\hline 88 UKR & 10000 & -2.9 & $-3.34 \%$ & 9.74 & 3.44 & 1.7 & 0.03 & 0.0087 & 42.9360 & $0.24 \%$ & $0.37 \%$ & $0.41 \%$ & $0.34 \%$ & $0.18 \%$ \\
\hline 89 XEE & 10000 & -2.9 & $-3.34 \%$ & 9.74 & 3.44 & 1.7 & 0.03 & 0.0087 & 42.9360 & $0.24 \%$ & $0.37 \%$ & $0.41 \%$ & $0.34 \%$ & $0.18 \%$ \\
\hline 90 XER & 10000 & -2.4 & $-3.17 \%$ & 8.76 & 2.85 & 2.54 & 0.05 & 0.0175 & 51.8246 & $0.35 \%$ & $0.60 \%$ & $0.76 \%$ & $0.81 \%$ & $0.77 \%$ \\
\hline $91 \mid \mathrm{KAZ}$ & 10000 & -2.9 & $-3.86 \%$ & 7.88 & 3.9 & 0.92 & 0.08 & 0.0205 & 37.8718 & $0.41 \%$ & $0.72 \%$ & $0.93 \%$ & $1.04 \%$ & $1.05 \%$ \\
\hline 92 KGZ & 10000 & -4.2 & $-6.68 \%$ & 2.88 & 3.63 & 1.54 & 0.06 & 0.0165 & 40.6887 & $0.91 \%$ & $1.72 \%$ & $2.44 \%$ & $3.05 \%$ & $3.57 \%$ \\
\hline 93 XSU & 10000 & -2.9 & $-3.86 \%$ & 7.88 & 3.9 & 0.92 & 0.08 & 0.0205 & 37.8718 & $0.41 \%$ & $0.72 \%$ & $0.93 \%$ & $1.04 \%$ & $1.05 \%$ \\
\hline 94 ARM & 10000 & -4.2 & $-5.14 \%$ & 8.1 & 3.44 & 1.79 & -0.07 & -0.0203 & 42.9360 & $0.40 \%$ & $0.70 \%$ & $0.90 \%$ & $1.00 \%$ & $1.00 \%$ \\
\hline 95 AZE & 1500 & -4.3 & $0.09 \%$ & 13.44 & 3.09 & 1.25 & 0 & 0.0000 & 47.7994 & $-0.81 \%$ & $-2.29 \%$ & $-4.43 \%$ & $-7.23 \%$ & $-10.69 \%$ \\
\hline 96 GEO & 10000 & -3.9 & $-4.55 \%$ & 8.96 & 3.3 & 2.32 & -0.04 & -0.0121 & 44.7576 & $0.32 \%$ & $0.53 \%$ & $0.65 \%$ & $0.67 \%$ & $0.59 \%$ \\
\hline $97 \mathrm{BHR}$ & 6000 & -6.8 & $0.44 \%$ & 25.72 & 3.21 & 0.2 & 0.01 & 0.0031 & 46.0125 & $-2.24 \%$ & $-4.65 \%$ & $-7.23 \%$ & $-9.97 \%$ & $-12.87 \%$ \\
\hline $98 \mid$ IRN & 3000 & -5.6 & $-0.03 \%$ & 16.74 & 3.51 & 0.63 & -0.03 & -0.0085 & 42.0798 & $-1.54 \%$ & $-3.41 \%$ & $-5.62 \%$ & $-8.15 \%$ & $-11.02 \%$ \\
\hline 99 ISR & 4000 & -5.9 & $0.15 \%$ & 19.36 & 3.05 & 0.4 & -0.07 & -0.0230 & 48.4262 & $-1.77 \%$ & $-3.79 \%$ & $-6.06 \%$ & $-8.58 \%$ & $-11.34 \%$ \\
\hline 100/JOR & 4000 & -5.9 & $-0.36 \%$ & 18.56 & 3.39 & 0.28 & -0.04 & -0.0118 & 43.5693 & $-1.60 \%$ & $-3.45 \%$ & $-5.54 \%$ & $-7.89 \%$ & $-10.48 \%$ \\
\hline 101|KWT & 6000 & -6.3 & $0.52 \%$ & 24.73 & 3.55 & 0.24 & 0.01 & 0.0028 & 41.6056 & $-2.10 \%$ & $-4.36 \%$ & $-6.78 \%$ & $-9.37 \%$ & $-12.13 \%$ \\
\hline 102 OMN & 6000 & -7.6 & $0.09 \%$ & 26.71 & 2.86 & 0.25 & 0.05 & 0.0175 & 51.6434 & $-2.39 \%$ & $-4.94 \%$ & $-7.66 \%$ & $-10.54 \%$ & $-13.59 \%$ \\
\hline 103 QAT & 6000 & -7.0 & $0.64 \%$ & 26.45 & 3.29 & 0.18 & 0.01 & 0.0030 & 44.8936 & $-2.37 \%$ & $-4.90 \%$ & $-7.60 \%$ & $-10.47 \%$ & $-13.50 \%$ \\
\hline 104 SAU & 6000 & -7.2 & $-0.85 \%$ & 23.91 & 3.56 & 0.23 & 0.03 & 0.0084 & 41.4888 & $-1.96 \%$ & $-4.08 \%$ & $-6.38 \%$ & $-8.83 \%$ & $-11.45 \%$ \\
\hline 106 TUR & 500 & -4.5 & $0.58 \%$ & 11.99 & 3.33 & 1.79 & -0.17 & -0.0511 & 44.3544 & $0.29 \%$ & $-1.41 \%$ & $-5.09 \%$ & $-10.77 \%$ & $-18.42 \%$ \\
\hline 105 ARE & 6000 & -7.0 & $0.97 \%$ & 27.28 & 3.28 & 0.19 & 0.01 & 0.0030 & 45.0305 & $-2.51 \%$ & $-5.18 \%$ & $-8.01 \%$ & $-11.01 \%$ & $-14.18 \%$ \\
\hline 107|XWS & 4000 & -5.9 & $-0.36 \%$ & 18.56 & 3.39 & 0.28 & -0.04 & -0.0118 & 43.5693 & $-1.60 \%$ & $-3.45 \%$ & $-5.54 \%$ & $-7.89 \%$ & $-10.48 \%$ \\
\hline 108 EGY & 5000 & -6.6 & $0.00 \%$ & 22.27 & 3.18 & 0.09 & -0.01 & -0.0031 & 46.4465 & $-2.00 \%$ & $-4.21 \%$ & $-6.61 \%$ & $-9.21 \%$ & $-12.01 \%$ \\
\hline 109 MAR & 4000 & -5.8 & $-0.50 \%$ & 18.32 & 3.2 & 0.53 & -0.12 & -0.0375 & 46.1563 & $-1.53 \%$ & $-3.30 \%$ & $-5.32 \%$ & $-7.60 \%$ & $-10.12 \%$ \\
\hline 110|TUN & 4000 & -5.5 & $0.41 \%$ & 19.14 & 2.98 & 0.46 & -0.07 & -0.0235 & 49.5638 & $-1.71 \%$ & $-3.67 \%$ & $-5.88 \%$ & $-8.34 \%$ & $-11.04 \%$ \\
\hline 111 XNF & 4000 & -5.5 & $0.41 \%$ & 19.14 & 2.98 & 0.46 & -0.07 & -0.0235 & 49.5638 & $-1.71 \%$ & $-3.67 \%$ & $-5.88 \%$ & $-8.34 \%$ & $-11.04 \%$ \\
\hline 112 BEN & 5000 & -9.7 & $-0.73 \%$ & 26.36 & 2.72 & 3.11 & 0.04 & 0.0147 & 54.3015 & $-2.78 \%$ & $-5.76 \%$ & $-8.95 \%$ & $-12.32 \%$ & $-15.90 \%$ \\
\hline 113 BFA & 5000 & -9.2 & $0.34 \%$ & 27.29 & 3.02 & 2.09 & 0.08 & 0.0265 & 48.9073 & $-2.99 \%$ & $-6.18 \%$ & $-9.57 \%$ & $-13.16 \%$ & $-16.94 \%$ \\
\hline 114 CMR & 3500 & -10.0 & $0.29 \%$ & 23.5 & 2.65 & 5.08 & 0.21 & 0.0792 & 55.7358 & $-3.15 \%$ & $-6.59 \%$ & $-10.31 \%$ & $-14.32 \%$ & $-18.61 \%$ \\
\hline 115 CIV & 4000 & -10.0 & $0.37 \%$ & 25.33 & 2.65 & 4.04 & 0.04 & 0.0151 & 55.7358 & $-3.22 \%$ & $-6.68 \%$ & $-10.39 \%$ & $-14.35 \%$ & $-18.56 \%$ \\
\hline 116 GHA & 4500 & -9.9 & $-0.16 \%$ & 26.17 & 2.59 & 3.5 & 0.03 & 0.0116 & 57.0270 & $-3.04 \%$ & $-6.30 \%$ & $-9.77 \%$ & $-13.47 \%$ & $-17.39 \%$ \\
\hline 117 GIN & 4000 & -9.6 & $0.92 \%$ & 25.41 & 2.81 & 3.54 & -0.03 & -0.0107 & 52.5623 & $-3.25 \%$ & $-6.75 \%$ & $-10.50 \%$ & $-14.50 \%$ & $-18.75 \%$ \\
\hline 118 NGA & 4000 & -9.8 & $0.88 \%$ & 25.63 & 2.73 & 3.2 & 0.09 & 0.0330 & 54.1026 & $-3.30 \%$ & $-6.85 \%$ & $-10.64 \%$ & $-14.68 \%$ & $-18.98 \%$ \\
\hline 119 SEN & 5500 & -8.8 & $0.17 \%$ & 27.88 & 2.75 & 0.99 & -0.02 & -0.0073 & 53.7091 & $-2.81 \%$ & $-5.79 \%$ & $-8.96 \%$ & $-12.31 \%$ & $-15.84 \%$ \\
\hline $120 \mathrm{TGO}$ & 4500 & -9.8 & $-0.03 \%$ & 26.21 & 2.65 & 3.46 & 0.01 & 0.0038 & 55.7358 & $-3.05 \%$ & $-6.33 \%$ & $-9.82 \%$ & $-13.53 \%$ & $-17.47 \%$ \\
\hline 121 XWF & 4500 & -9.8 & $-0.03 \%$ & 26.21 & 2.65 & 3.46 & 0.01 & 0.0038 & 55.7358 & $-3.05 \%$ & $-6.33 \%$ & $-9.82 \%$ & $-13.53 \%$ & $-17.47 \%$ \\
\hline 122 XCF & 4000 & -10.2 & $-0.48 \%$ & 24.37 & 2.79 & 4.02 & 0.26 & 0.0932 & 52.9391 & $-2.99 \%$ & $-6.23 \%$ & $-9.72 \%$ & $-13.45 \%$ & $-17.44 \%$ \\
\hline 123XXC & 3500 & -11.3 & $-0.68 \%$ & 24 & 2.44 & 1.78 & 0.23 & 0.0943 & 60.5328 & $-3.27 \%$ & $-6.82 \%$ & $-10.65 \%$ & $-14.77 \%$ & $-19.17 \%$ \\
\hline 124 ETH & 3500 & -9.8 & $-0.50 \%$ & 22.25 & 2.68 & 2.11 & 0.29 & 0.1082 & 55.1119 & $-2.80 \%$ & $-5.89 \%$ & $-9.26 \%$ & $-12.91 \%$ & $-16.85 \%$ \\
\hline $125 \mid$ KEN & 3500 & -11.3 & $-0.68 \%$ & 24 & 2.44 & 1.78 & 0.23 & 0.0943 & 60.5328 & $-3.27 \%$ & $-6.82 \%$ & $-10.65 \%$ & $-14.77 \%$ & $-19.17 \%$ \\
\hline 126|MDG & 4000 & -8.0 & $0.33 \%$ & 22.74 & 2.33 & 3.56 & -0.12 & -0.0515 & 63.3906 & $-2.53 \%$ & $-5.31 \%$ & $-8.34 \%$ & $-11.62 \%$ & $-15.14 \%$ \\
\hline 127|MWI & 3000 & -9.1 & $0.67 \%$ & 21.08 & 2.86 & 3.33 & -0.17 & -0.0594 & 51.6434 & $-2.91 \%$ & $-6.15 \%$ & $-9.72 \%$ & $-13.63 \%$ & $-17.86 \%$ \\
\hline 128 MUS & 5000 & -7.7 & $-0.32 \%$ & 24.3 & 1.77 & 2.88 & -0.11 & -0.0621 & 83.4463 & $-2.25 \%$ & $-4.70 \%$ & $-7.34 \%$ & $-10.19 \%$ & $-13.23 \%$ \\
\hline $129 \mathrm{MOZ}$ & 4500 & -8.1 & $-0.25 \%$ & 23.21 & 2.68 & 3 & -0.16 & -0.0597 & 55.1119 & $-2.39 \%$ & $-5.01 \%$ & $-7.84 \%$ & $-10.90 \%$ & $-14.18 \%$ \\
\hline 130|RWA & 2200 & -11.0 & $0.26 \%$ & 19.66 & 2.72 & 4.83 & 0.46 & 0.1691 & 54.3015 & $-3.30 \%$ & $-7.04 \%$ & $-11.24 \%$ & $-15.89 \%$ & $-21.00 \%$ \\
\hline 131 TZA & 3000 & -10.2 & $-0.35 \%$ & 21.31 & 2.63 & 3.26 & 0.08 & 0.0304 & 56.1597 & $-2.95 \%$ & $-6.24 \%$ & $-9.85 \%$ & $-13.80 \%$ & $-18.07 \%$ \\
\hline 132 UGA & 3000 & -11.1 & $-0.30 \%$ & 22.29 & 2.54 & 3.5 & 0.4 & 0.1575 & 58.1496 & $-3.26 \%$ & $-6.85 \%$ & $-10.78 \%$ & $-15.03 \%$ & $-19.61 \%$ \\
\hline 133 ZMB & 3000 & -9.1 & $0.63 \%$ & 20.97 & 3.07 & 3.45 & -0.12 & -0.0391 & 48.1107 & $-2.90 \%$ & $-6.13 \%$ & $-9.69 \%$ & $-13.58 \%$ & $-17.81 \%$ \\
\hline 134 ZWE & 3500 & -8.1 & $0.28 \%$ & 21.06 & 3.04 & 2.46 & -0.15 & -0.0493 & 48.5855 & $-2.51 \%$ & $-5.30 \%$ & $-8.37 \%$ & $-11.73 \%$ & $-15.38 \%$ \\
\hline 135 XEC & 4000 & -8.1 & $0.73 \%$ & 23.21 & 2.68 & 3 & -0.16 & -0.0597 & 55.1119 & $-2.69 \%$ & $-5.63 \%$ & $-8.82 \%$ & $-12.26 \%$ & $-15.95 \%$ \\
\hline 136 BWA & 4000 & -7.4 & $0.02 \%$ & 21.07 & 3.38 & 2.01 & -0.15 & -0.0444 & 43.6982 & $-2.22 \%$ & $-4.69 \%$ & $-7.41 \%$ & $-10.38 \%$ & $-13.60 \%$ \\
\hline 137NAM & 4000 & -7.3 & $0.27 \%$ & 21.35 & 3.17 & 1.49 & -0.12 & -0.0379 & 46.5931 & $-2.28 \%$ & $-4.80 \%$ & $-7.57 \%$ & $-10.60 \%$ & $-13.87 \%$ \\
\hline 138 ZAF & 2500 & -6.3 & $0.41 \%$ & 16.93 & 2.94 & 2.32 & -0.08 & -0.0272 & 50.2381 & $-1.85 \%$ & $-4.11 \%$ & $-6.75 \%$ & $-9.80 \%$ & $-13.25 \%$ \\
\hline 139 XSC & 2500 & -6.3 & $0.41 \%$ & 16.93 & 2.94 & 2.32 & -0.08 & -0.0272 & 50.2381 & $-1.85 \%$ & $-4.11 \%$ & $-6.75 \%$ & $-9.80 \%$ & $-13.25 \%$ \\
\hline 140 XTW & 2000 & -4.3 & $-0.25 \%$ & 14 & 2.95 & 1.8 & -0.25 & -0.0847 & 50.0678 & $-0.87 \%$ & $-2.23 \%$ & $-4.09 \%$ & $-6.44 \%$ & $-9.29 \%$ \\
\hline
\end{tabular}


Table A5. Adjustment factors for regional temperature changes.

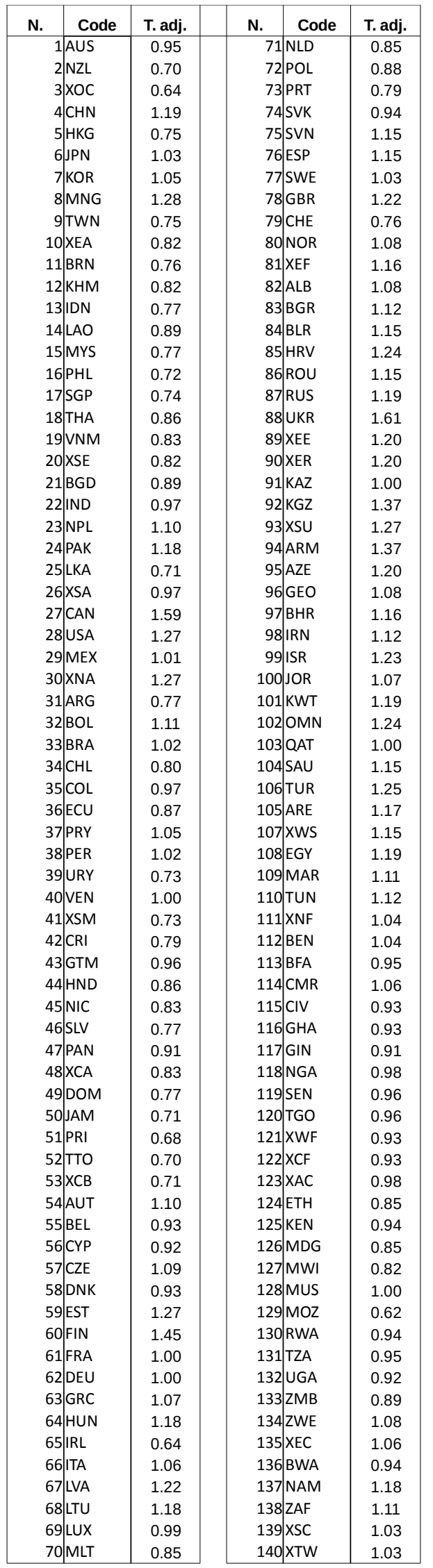


Table A6-1. Heat impacts on labor productivity, by sector (percentage change). Values below $-10 \%$ in red.

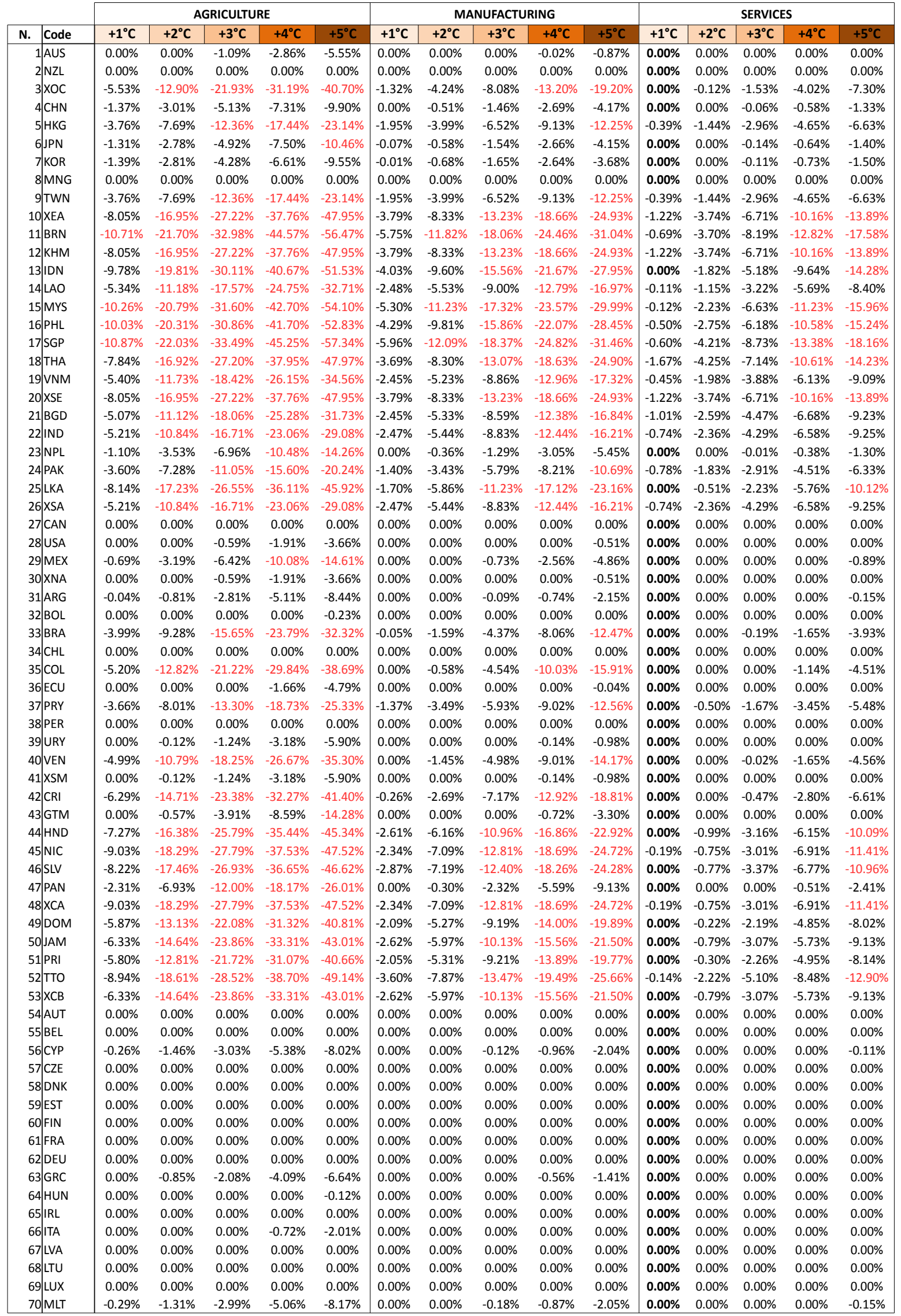


Table A6-2. Heat impacts on labor productivity, by sector (percentage change). Values below $-10 \%$ in red.

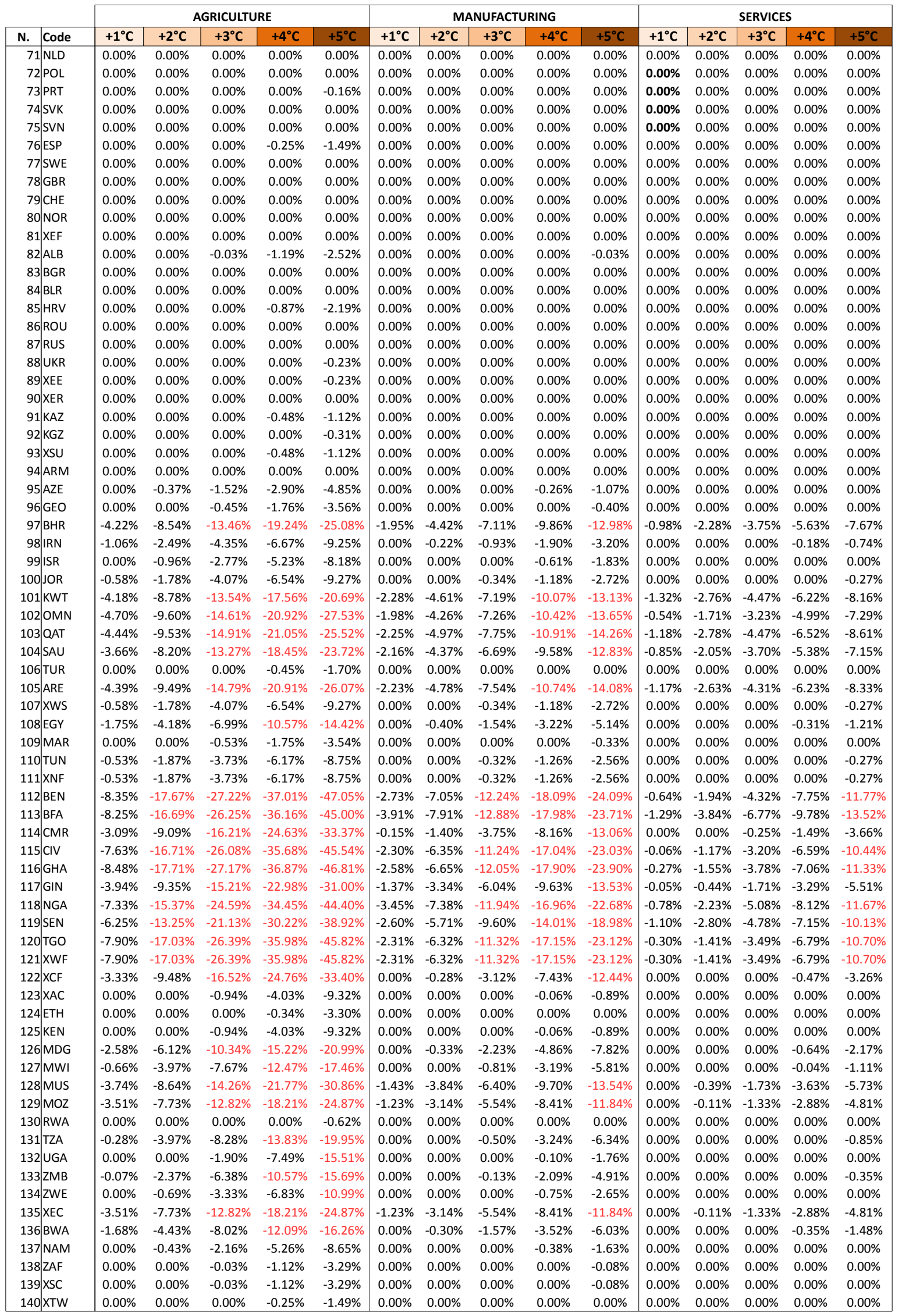


Table A7. Human health: percentage variation of labor productivity for $+1^{\circ} \mathrm{C}$.

\begin{tabular}{|c|c|c|c|}
\hline Code & $\begin{array}{c}\% \text { var. in labor } \\
\text { productivity }\end{array}$ & Code & \begin{tabular}{|c|}
$\begin{array}{c}\% \text { var. in labor } \\
\text { productivity }\end{array}$ \\
\end{tabular} \\
\hline 1 AUS & -0.1489 & $71 \mid$ NLD & -0.0337 \\
\hline $2 \mathrm{NZL}$ & -0.1489 & 72 POL & -0.0315 \\
\hline $3 \times O C$ & -0.2423 & 73 PRT & -0.0337 \\
\hline $4 \mathrm{CHN}$ & -0.5378 & 74 SVK & -0.0337 \\
\hline 5 HKG & -0.5378 & 75 SVN & -0.0337 \\
\hline 6JPN & -0.0631 & 76 ESP & -0.0337 \\
\hline 7 KOR & -0.0631 & 77SWE & -0.0337 \\
\hline 8 MNG & -0.5378 & $78 \mathrm{GBR}$ & -0.0337 \\
\hline 9 TWN & -0.5378 & $79 \mathrm{CHE}$ & -0.0337 \\
\hline 10XEA & -0.1315 & 80 NOR & -0.0337 \\
\hline 11 BRN & -0.1840 & 81 XEF & -0.0527 \\
\hline $12 \mathrm{KHM}$ & -0.1315 & $82 \mid A L B$ & -0.0697 \\
\hline 13 IDN & -0.1453 & 83 BGR & -0.0697 \\
\hline 14 LAO & -0.1315 & 84 BLR & -0.0697 \\
\hline 15 MYS & -0.1453 & $85 \mathrm{HRV}$ & -0.0315 \\
\hline $16 \mathrm{PHL}$ & -0.1315 & 86 ROU & -0.0315 \\
\hline 17) SGP & -0.2375 & 87|RUS & -0.0589 \\
\hline 18 THA & -0.1386 & 88 UKR & -0.0589 \\
\hline 19VNM & -0.1315 & $89 \mid$ XEE & -0.0697 \\
\hline 20 XSE & -0.1315 & 90|XER & -0.0337 \\
\hline $21 \mathrm{BGD}$ & -0.1386 & 91 KAZ & -0.0589 \\
\hline 22 IND & -0.7468 & 92 KGZ & -0.0589 \\
\hline $23 \mathrm{NPL}$ & -0.7468 & 93 XSU & -0.0589 \\
\hline 24PAK & -0.1386 & 94ARM & -0.0589 \\
\hline 25 LKA & -0.7468 & 95 AZE & -0.0589 \\
\hline 26 XSA & -0.1386 & 96 GEO & -0.0589 \\
\hline 27 CAN & 0.0000 & $97 \mathrm{BHR}$ & -0.5921 \\
\hline 28 USA & -0.1447 & 98 IRN & -0.2128 \\
\hline 29|MEX & -0.2412 & 99 ISR & -0.6843 \\
\hline 30XNA & -0.2349 & 100/JOR & -0.5921 \\
\hline 31 ARG & -0.2191 & 101 KWT & -0.5921 \\
\hline $32 \mathrm{BOL}$ & -0.1131 & $102 \mathrm{OMN}$ & -0.5921 \\
\hline 33 BRA & -0.2191 & 103QAT & -0.5921 \\
\hline $34 \mathrm{CHL}$ & -0.2191 & 104 SAU & -0.5921 \\
\hline $35 \mathrm{COL}$ & -0.1131 & 106|TUR & -0.3050 \\
\hline 36 ECU & -0.1131 & 105ARE & -0.5921 \\
\hline 37|PRY & -0.1131 & 107|XWS & -0.1990 \\
\hline 38 PER & -0.1131 & 108 EGY & -0.3657 \\
\hline 39 URY & -0.2191 & 109 MAR & -0.4044 \\
\hline 40 VEN & -0.1269 & 110TUN & -0.4044 \\
\hline 41 XSM & -0.1131 & 111 XNF & -0.4044 \\
\hline $42 \mathrm{CRI}$ & -0.2412 & 112 BEN & -0.6308 \\
\hline 43 GTM & -0.1352 & $113 \mathrm{BFA}$ & -0.6308 \\
\hline 44HND & -0.1352 & $114 \mathrm{CMR}$ & -0.6308 \\
\hline 45 NIC & -0.1352 & 115 CIV & -0.6308 \\
\hline 46SLV & -0.1352 & $116 \mathrm{GHA}$ & -0.6308 \\
\hline 47|PAN & -0.1352 & 117 GIN & -0.6308 \\
\hline $48 \times C A$ & -0.1352 & 118 NGA & -0.6308 \\
\hline 49DOM & -0.1352 & 119SEN & -0.6308 \\
\hline 50JAM & -0.1352 & 120|TGO & -0.6308 \\
\hline 51|PRI & -0.1352 & 121 XWF & -0.6308 \\
\hline 52 & -0.1352 & 122 XCF & -0.6308 \\
\hline $53 \times C B$ & -0.1352 & 123 XAC & -0.6308 \\
\hline 54AUT & -0.0337 & 124 ЕTH & -0.6308 \\
\hline $55 \mathrm{BEL}$ & -0.0337 & 125 KEN & -0.6308 \\
\hline 56 CYP & -0.0337 & 126/MDG & -0.6308 \\
\hline 57CZE & -0.0315 & 127/MWI & -0.6308 \\
\hline 58 DNK & -0.0337 & $128 \mathrm{MUS}$ & -0.5783 \\
\hline 59 EST & -0.0315 & $129 \mid \mathrm{MOZ}$ & -0.6308 \\
\hline 60 FIN & -0.0337 & 130RWA & -0.6308 \\
\hline 61 FRA & -0.0337 & 131|TZA & -0.6308 \\
\hline 62 DEU & -0.0337 & 132 UGA & -0.6308 \\
\hline $63 \mathrm{GRC}$ & -0.0337 & 133 ZMB & -0.6308 \\
\hline 64HUN & -0.0315 & 134/ZWE & -0.6308 \\
\hline 65 IRL & -0.0337 & 135 XEC & -0.6308 \\
\hline 66 ITA & -0.0337 & 136BWA & -0.6308 \\
\hline 67)LVA & -0.0315 & 137/NAM & -0.6308 \\
\hline 68 LTU & -0.0315 & $138 \mathrm{ZAF}$ & -0.6308 \\
\hline 69 LUX & -0.0337 & 139 XSC & -0.6308 \\
\hline 70 MLT & -0.0337 & 140 XTW & -0.5783 \\
\hline
\end{tabular}


Table A8. Tourism: changes in net foreign currency inflows (relative to 2011 GDP). Negative values in red.

\begin{tabular}{|c|c|c|c|c|c|c|c|c|c|c|c|c|}
\hline Code & $+1^{\circ} \mathrm{C}$ & $+2^{\circ} \mathrm{C}$ & $+3^{\circ} \mathrm{C}$ & $+4^{\circ} \mathrm{C}$ & $+5^{\circ} \mathrm{C}$ & N. & Code & $+1^{\circ} \mathrm{C}$ & $+2^{\circ} \mathrm{C}$ & $+3^{\circ} \mathrm{C}$ & $+4^{\circ} \mathrm{C}$ & $+5^{\circ} \mathrm{C}$ \\
\hline 1 AUS & $-0.14 \%$ & $-0.31 \%$ & $-0.50 \%$ & $-0.71 \%$ & $-0.94 \%$ & & NLD & $0.29 \%$ & $0.55 \%$ & $0.76 \%$ & $0.93 \%$ & $1.07 \%$ \\
\hline 2 NZL & $0.06 \%$ & $0.12 \%$ & $0.18 \%$ & $0.25 \%$ & $0.33 \%$ & 72 & POL & $0.33 \%$ & $0.65 \%$ & $0.95 \%$ & $1.23 \%$ & $1.48 \%$ \\
\hline $3 \mathrm{XOC}$ & $0.00 \%$ & $0.00 \%$ & $0.00 \%$ & $0.00 \%$ & $0.00 \%$ & 73 & PRT & $-0.25 \%$ & $-0.50 \%$ & $-0.76 \%$ & $-1.01 \%$ & $-1.24 \%$ \\
\hline $4 \mathrm{CHN}$ & $0.05 \%$ & $0.08 \%$ & $0.09 \%$ & $0.08 \%$ & $0.06 \%$ & 74 & SVK & $0.30 \%$ & $0.71 \%$ & $1.23 \%$ & $1.84 \%$ & $2.55 \%$ \\
\hline 5 HKG & $-1.77 \%$ & $-3.53 \%$ & $-5.25 \%$ & $-6.93 \%$ & $-8.57 \%$ & 75 & SVN & $0.21 \%$ & $0.65 \%$ & $1.30 \%$ & $2.14 \%$ & $3.16 \%$ \\
\hline 6 JPN & $0.01 \%$ & $0.02 \%$ & $0.02 \%$ & $0.02 \%$ & $0.01 \%$ & 76 & ESP & $-0.13 \%$ & $-0.32 \%$ & $-0.55 \%$ & $-0.82 \%$ & $-1.12 \%$ \\
\hline 7 KOR & $0.09 \%$ & $0.16 \%$ & $0.21 \%$ & $0.24 \%$ & $0.26 \%$ & 77 & SWE & $0.60 \%$ & $1.17 \%$ & $1.72 \%$ & $2.23 \%$ & $2.71 \%$ \\
\hline 8 MNG & $-1.51 \%$ & $-1.18 \%$ & $0.95 \%$ & $4.83 \%$ & $10.47 \%$ & 78 & GBR & $0.26 \%$ & $0.47 \%$ & $0.64 \%$ & $0.77 \%$ & $0.86 \%$ \\
\hline 9 TWN & $-0.69 \%$ & $-1.39 \%$ & $-2.09 \%$ & $-2.80 \%$ & $-3.51 \%$ & 79 & CHE & $0.52 \%$ & $1.01 \%$ & $1.47 \%$ & $1.89 \%$ & $2.26 \%$ \\
\hline 10XEA & $0.00 \%$ & $0.00 \%$ & $0.00 \%$ & $0.00 \%$ & $0.00 \%$ & 80 & NOR & $0.52 \%$ & $1.00 \%$ & $1.44 \%$ & $1.87 \%$ & $2.27 \%$ \\
\hline $11 \mid$ BRN & $-1.88 \%$ & $-2.75 \%$ & $-2.68 \%$ & $-1.68 \%$ & $0.21 \%$ & 81 & XEF & $0.00 \%$ & $0.00 \%$ & $0.00 \%$ & $0.00 \%$ & $0.00 \%$ \\
\hline 12 KHM & $-5.21 \%$ & $-8.74 \%$ & $-10.65 \%$ & $-11.02 \%$ & $-9.92 \%$ & 82 & ALB & $-1.57 \%$ & $-2.18 \%$ & $-1.85 \%$ & $-0.59 \%$ & $1.63 \%$ \\
\hline 13 IDN & $-0.26 \%$ & $-0.50 \%$ & $-0.71 \%$ & $-0.90 \%$ & $-1.08 \%$ & 83 & $B G R$ & $0.20 \%$ & $0.57 \%$ & $1.08 \%$ & $1.72 \%$ & $2.49 \%$ \\
\hline 14 LAO & $-3.93 \%$ & $-5.82 \%$ & $-5.76 \%$ & $-3.81 \%$ & $0.01 \%$ & 84 & $B L R$ & $-0.20 \%$ & $-0.15 \%$ & $0.15 \%$ & $0.68 \%$ & $1.44 \%$ \\
\hline 15 MYS & $-1.63 \%$ & $-3.10 \%$ & $-4.44 \%$ & $-5.66 \%$ & $-6.81 \%$ & 85 & HRV & $-0.15 \%$ & $-0.28 \%$ & $-0.42 \%$ & $-0.54 \%$ & $-0.62 \%$ \\
\hline 16 PHL & $-0.59 \%$ & $-1.11 \%$ & $-1.59 \%$ & $-2.03 \%$ & $-2.46 \%$ & 86 & ROU & $0.02 \%$ & $0.11 \%$ & $0.26 \%$ & $0.47 \%$ & $0.74 \%$ \\
\hline $17 \mathrm{SGP}$ & $-2.04 \%$ & $-4.01 \%$ & $-5.92 \%$ & $-7.84 \%$ & $-9.84 \%$ & 87 & RUS & $0.43 \%$ & $0.82 \%$ & $1.21 \%$ & $1.58 \%$ & $1.95 \%$ \\
\hline 18 THA & $-1.72 \%$ & $-3.22 \%$ & $-4.50 \%$ & $-5.61 \%$ & $-6.55 \%$ & 88 & UKR & $0.29 \%$ & $0.61 \%$ & $0.94 \%$ & $1.30 \%$ & $1.67 \%$ \\
\hline 19 VNM & $-0.82 \%$ & $-1.55 \%$ & $-2.19 \%$ & $-2.77 \%$ & $-3.29 \%$ & 89 & XEE & $0.00 \%$ & $0.00 \%$ & $0.00 \%$ & $0.00 \%$ & $0.00 \%$ \\
\hline 20XSE & $0.00 \%$ & $0.00 \%$ & $0.00 \%$ & $0.00 \%$ & $0.00 \%$ & 90 & XER & $0.00 \%$ & $0.00 \%$ & $0.00 \%$ & $0.00 \%$ & $0.00 \%$ \\
\hline $21 \mid B G D$ & $-0.25 \%$ & $-0.37 \%$ & $-0.34 \%$ & $-0.18 \%$ & $0.11 \%$ & 91 & KAZ & $0.05 \%$ & $0.16 \%$ & $0.34 \%$ & $0.58 \%$ & $0.88 \%$ \\
\hline $22 \mid$ IND & $-0.21 \%$ & $-0.40 \%$ & $-0.58 \%$ & $-0.76 \%$ & $-0.93 \%$ & 92 & KGZ & $-3.09 \%$ & $-3.63 \%$ & $-1.76 \%$ & $2.45 \%$ & $9.00 \%$ \\
\hline $23 \mathrm{NPL}$ & $-1.46 \%$ & $-2.08 \%$ & $-1.88 \%$ & $-0.88 \%$ & $0.92 \%$ & 93 & XSU & $0.00 \%$ & $0.00 \%$ & $0.00 \%$ & $0.00 \%$ & $0.00 \%$ \\
\hline $24 \mid$ PAK & $-0.15 \%$ & $-0.24 \%$ & $-0.25 \%$ & $-0.20 \%$ & $-0.08 \%$ & 94 & ARM & $-1.48 \%$ & $-1.45 \%$ & $0.02 \%$ & $2.88 \%$ & $7.12 \%$ \\
\hline 25 LKA & $-0.72 \%$ & $-1.14 \%$ & $-1.29 \%$ & $-1.17 \%$ & $-0.78 \%$ & 95 & AZE & $-0.23 \%$ & $-0.27 \%$ & $-0.13 \%$ & $0.20 \%$ & $0.71 \%$ \\
\hline 26 XSA & $0.00 \%$ & $0.00 \%$ & $0.00 \%$ & $0.00 \%$ & $0.00 \%$ & 96 & GEO & $-1.65 \%$ & $-2.28 \%$ & $-1.92 \%$ & $-0.59 \%$ & $1.72 \%$ \\
\hline 27 CAN & $0.40 \%$ & $0.76 \%$ & $1.10 \%$ & $1.42 \%$ & $1.71 \%$ & 97 & BHR & $-1.67 \%$ & $-2.74 \%$ & $-3.23 \%$ & $-3.18 \%$ & $-2.61 \%$ \\
\hline 28 USA & $0.06 \%$ & $0.10 \%$ & $0.12 \%$ & $0.11 \%$ & $0.09 \%$ & 98 & IRN & $-0.05 \%$ & $-0.08 \%$ & $-0.08 \%$ & $-0.06 \%$ & $-0.02 \%$ \\
\hline 29 MEX & $-0.15 \%$ & $-0.29 \%$ & $-0.42 \%$ & $-0.54 \%$ & $-0.65 \%$ & 99 & ISR & $-0.29 \%$ & $-0.54 \%$ & $-0.76 \%$ & $-0.92 \%$ & $-1.04 \%$ \\
\hline 30 XNA & $0.00 \%$ & $0.00 \%$ & $0.00 \%$ & $0.00 \%$ & $0.00 \%$ & 100 & JOR & $-1.81 \%$ & $-3.16 \%$ & $-4.05 \%$ & $-4.46 \%$ & $-4.36 \%$ \\
\hline 31 ARG & $-0.09 \%$ & $-0.18 \%$ & $-0.25 \%$ & $-0.31 \%$ & $-0.35 \%$ & 101 & KWT & $-0.53 \%$ & $-1.04 \%$ & $-1.54 \%$ & $-2.05 \%$ & $-2.60 \%$ \\
\hline $32 \mathrm{BOL}$ & $-1.10 \%$ & $-1.53 \%$ & $-1.33 \%$ & $-0.50 \%$ & $0.95 \%$ & 102 & OMN & $-0.75 \%$ & $-1.27 \%$ & $-1.56 \%$ & $-1.64 \%$ & $-1.53 \%$ \\
\hline 33 BRA & $-0.11 \%$ & $-0.22 \%$ & $-0.33 \%$ & $-0.45 \%$ & $-0.58 \%$ & 103 & QAT & $-0.37 \%$ & $-0.64 \%$ & $-0.83 \%$ & $-0.94 \%$ & $-0.98 \%$ \\
\hline $34 \mathrm{CHL}$ & $-0.05 \%$ & $-0.04 \%$ & $0.00 \%$ & $0.09 \%$ & $0.22 \%$ & 104 & SAU & $-0.44 \%$ & $-0.87 \%$ & $-1.30 \%$ & $-1.74 \%$ & $-2.21 \%$ \\
\hline $35 \mathrm{COL}$ & $-0.26 \%$ & $-0.48 \%$ & $-0.65 \%$ & $-0.77 \%$ & $-0.86 \%$ & 106 & TUR & $0.03 \%$ & $0.03 \%$ & $-0.01 \%$ & $-0.08 \%$ & $-0.18 \%$ \\
\hline 36 ECU & $-0.44 \%$ & $-0.67 \%$ & $-0.70 \%$ & $-0.54 \%$ & $-0.18 \%$ & 105 & ARE & $-0.97 \%$ & $-1.92 \%$ & $-2.87 \%$ & $-3.87 \%$ & $-4.94 \%$ \\
\hline $37 \mid$ PRY & $-1.12 \%$ & $-1.59 \%$ & $-1.43 \%$ & $-0.65 \%$ & $0.74 \%$ & 107 & XWS & $0.00 \%$ & $0.00 \%$ & $0.00 \%$ & $0.00 \%$ & $0.00 \%$ \\
\hline 38 PER & $-0.18 \%$ & $-0.28 \%$ & $-0.31 \%$ & $-0.27 \%$ & $-0.16 \%$ & 108 & EGY & $-0.59 \%$ & $-1.11 \%$ & $-1.55 \%$ & $-1.91 \%$ & $-2.18 \%$ \\
\hline 39 URY & $-0.74 \%$ & $-1.19 \%$ & $-1.36 \%$ & $-1.24 \%$ & $-0.83 \%$ & 109 & MAR & $-0.69 \%$ & $-1.30 \%$ & $-1.82 \%$ & $-2.23 \%$ & $-2.50 \%$ \\
\hline 40 VEN & $-0.16 \%$ & $-0.27 \%$ & $-0.35 \%$ & $-0.38 \%$ & $-0.38 \%$ & 110 & TUN & $-0.85 \%$ & $-1.38 \%$ & $-1.59 \%$ & $-1.50 \%$ & $-1.07 \%$ \\
\hline $41 \mid$ XSM & $0.00 \%$ & $0.00 \%$ & $0.00 \%$ & $0.00 \%$ & $0.00 \%$ & 111 & XNF & $0.00 \%$ & $0.00 \%$ & $0.00 \%$ & $0.00 \%$ & $0.00 \%$ \\
\hline 42 CRI & $-1.51 \%$ & $-2.56 \%$ & $-3.14 \%$ & $-3.28 \%$ & $-2.98 \%$ & 112 & BEN & $-3.88 \%$ & $-5.43 \%$ & $-4.77 \%$ & $-1.95 \%$ & $3.01 \%$ \\
\hline 43 GTM & $-0.87 \%$ & $-1.41 \%$ & $-1.62 \%$ & $-1.51 \%$ & $-1.08 \%$ & 113 & BFA & $-2.55 \%$ & $-3.51 \%$ & $-2.93 \%$ & $-0.88 \%$ & $2.65 \%$ \\
\hline 44 HND & $-2.15 \%$ & $-3.32 \%$ & $-3.57 \%$ & $-2.93 \%$ & $-1.40 \%$ & 114 & CMR & $-1.32 \%$ & $-2.00 \%$ & $-2.07 \%$ & $-1.55 \%$ & $-0.45 \%$ \\
\hline 45 NIC & $-3.36 \%$ & $-5.01 \%$ & $-5.03 \%$ & $-3.47 \%$ & $-0.35 \%$ & 115 & CIV & $-1.28 \%$ & $-1.88 \%$ & $-1.84 \%$ & $-1.17 \%$ & $0.11 \%$ \\
\hline 46 SLV & $-1.01 \%$ & $-1.50 \%$ & $-1.50 \%$ & $-1.02 \%$ & $-0.08 \%$ & 116 & GHA & $-1.05 \%$ & $-1.65 \%$ & $-1.84 \%$ & $-1.64 \%$ & $-1.04 \%$ \\
\hline 47 PAN & $-3.02 \%$ & $-5.38 \%$ & $-7.11 \%$ & $-8.24 \%$ & $-8.80 \%$ & 117 & GIN & $-4.86 \%$ & $-6.45 \%$ & $-4.94 \%$ & $-0.40 \%$ & $7.16 \%$ \\
\hline 48 XCA & $0.00 \%$ & $0.00 \%$ & $0.00 \%$ & $0.00 \%$ & $0.00 \%$ & 118 & NGA & $-0.23 \%$ & $-0.44 \%$ & $-0.64 \%$ & $-0.85 \%$ & $-1.08 \%$ \\
\hline 49 DOM & $-1.83 \%$ & $-3.23 \%$ & $-4.21 \%$ & $-4.78 \%$ & $-4.95 \%$ & 119 & SEN & $-2.43 \%$ & $-3.63 \%$ & $-3.68 \%$ & $-2.60 \%$ & $-0.43 \%$ \\
\hline 50 JAM & $-4.23 \%$ & $-7.04 \%$ & $-8.49 \%$ & $-8.60 \%$ & $-7.42 \%$ & 120 & TGO & $-6.91 \%$ & $-9.38 \%$ & $-7.63 \%$ & $-1.76 \%$ & $8.20 \%$ \\
\hline $51 \mid \mathrm{PRI}$ & $-0.44 \%$ & $-0.69 \%$ & $-0.78 \%$ & $-0.70 \%$ & $-0.46 \%$ & 121 & XWF & $0.00 \%$ & $0.00 \%$ & $0.00 \%$ & $0.00 \%$ & $0.00 \%$ \\
\hline \begin{tabular}{l|l|l}
52 & тто
\end{tabular} & $-1.45 \%$ & $-2.17 \%$ & $-2.18 \%$ & $-1.52 \%$ & $-0.18 \%$ & 122 & XCF & $-0.41 \%$ & $-0.56 \%$ & $-0.47 \%$ & $-0.13 \%$ & $0.44 \%$ \\
\hline $53 \times C B$ & $-1.49 \%$ & $-2.70 \%$ & $-3.66 \%$ & $-4.37 \%$ & $-4.85 \%$ & 123 & XAC & $0.00 \%$ & $0.00 \%$ & $0.00 \%$ & $0.00 \%$ & $0.00 \%$ \\
\hline 54 AUT & $0.71 \%$ & $1.37 \%$ & $1.98 \%$ & $2.52 \%$ & $2.98 \%$ & 124 & ETH & $-1.00 \%$ & $-1.48 \%$ & $-1.48 \%$ & $-0.98 \%$ & $0.01 \%$ \\
\hline $55 \mathrm{BEL}$ & $0.48 \%$ & $0.90 \%$ & $1.25 \%$ & $1.55 \%$ & $1.79 \%$ & 125 & KEN & $-1.02 \%$ & $-1.57 \%$ & $-1.66 \%$ & $-1.28 \%$ & $-0.45 \%$ \\
\hline 56 CYP & $-1.88 \%$ & $-3.22 \%$ & $-4.00 \%$ & $-4.22 \%$ & $-3.86 \%$ & 126 & MDG & $-2.84 \%$ & $-4.01 \%$ & $-3.59 \%$ & $-1.62 \%$ & $1.89 \%$ \\
\hline 57 CZE & $0.47 \%$ & $0.96 \%$ & $1.44 \%$ & $1.91 \%$ & $2.37 \%$ & 127 & MWI & $-4.43 \%$ & $-5.93 \%$ & $-4.63 \%$ & $-0.60 \%$ & $6.15 \%$ \\
\hline 58 DNK & $0.68 \%$ & $1.29 \%$ & $1.85 \%$ & $2.34 \%$ & $2.78 \%$ & 128 & MUS & $-4.35 \%$ & $-7.13 \%$ & $-8.38 \%$ & $-8.13 \%$ & $-6.40 \%$ \\
\hline 59 EST & $0.07 \%$ & $0.79 \%$ & $2.11 \%$ & $4.00 \%$ & $6.44 \%$ & 129 & MOZ & $-2.30 \%$ & $-3.29 \%$ & $-3.02 \%$ & $-1.52 \%$ & $1.18 \%$ \\
\hline $60 \mathrm{FIN}$ & $0.43 \%$ & $0.90 \%$ & $1.40 \%$ & $1.92 \%$ & $2.48 \%$ & 130 & RWA & $-4.15 \%$ & $-5.74 \%$ & $-4.89 \%$ & $-1.65 \%$ & $3.99 \%$ \\
\hline $61 \mid$ FRA & $0.16 \%$ & $0.28 \%$ & $0.35 \%$ & $0.38 \%$ & $0.37 \%$ & 131 & TZA & $-1.92 \%$ & $-3.16 \%$ & $-3.75 \%$ & $-3.69 \%$ & $-2.99 \%$ \\
\hline 62 DEU & $0.31 \%$ & $0.57 \%$ & $0.79 \%$ & $0.97 \%$ & $1.11 \%$ & 132 & UGA & $-2.40 \%$ & $-3.75 \%$ & $-4.07 \%$ & $-3.39 \%$ & $-1.70 \%$ \\
\hline 63 GRC & $-0.35 \%$ & $-0.70 \%$ & $-1.06 \%$ & $-1.40 \%$ & $-1.71 \%$ & 133 & ZMB & $-1.37 \%$ & $-1.87 \%$ & $-1.56 \%$ & $-0.44 \%$ & $1.48 \%$ \\
\hline $64 \mathrm{HUN}$ & $0.30 \%$ & $0.61 \%$ & $0.95 \%$ & $1.30 \%$ & $1.68 \%$ & 134 & ZWE & $-2.45 \%$ & $-3.42 \%$ & $-2.96 \%$ & $-1.12 \%$ & $2.12 \%$ \\
\hline 65 IRL & $0.23 \%$ & $0.47 \%$ & $0.72 \%$ & $0.96 \%$ & $1.22 \%$ & 135 & XEC & $0.00 \%$ & $0.00 \%$ & $0.00 \%$ & $0.00 \%$ & $0.00 \%$ \\
\hline 66 |TA & $0.04 \%$ & $0.03 \%$ & $-0.00 \%$ & $-0.07 \%$ & $-0.16 \%$ & 136 & BWA & $-1.63 \%$ & $-2.18 \%$ & $-1.71 \%$ & $-0.23 \%$ & $2.25 \%$ \\
\hline 67 LVA & $-0.23 \%$ & $0.05 \%$ & $0.83 \%$ & $2.07 \%$ & $3.77 \%$ & 137 & NAM & $-2.41 \%$ & $-3.51 \%$ & $-3.36 \%$ & $-1.97 \%$ & $0.65 \%$ \\
\hline 68 LTU & $0.00 \%$ & $0.34 \%$ & $0.98 \%$ & $1.90 \%$ & $3.11 \%$ & 138 & ZAF & $-0.18 \%$ & $-0.35 \%$ & $-0.52 \%$ & $-0.67 \%$ & $-0.81 \%$ \\
\hline 69 LUX & $0.88 \%$ & $1.85 \%$ & $2.88 \%$ & $3.96 \%$ & $5.09 \%$ & 139 & XSC & $0.00 \%$ & $0.00 \%$ & $0.00 \%$ & $0.00 \%$ & $0.00 \%$ \\
\hline 70|MLT & $-3.74 \%$ & $-5.83 \%$ & $-6.30 \%$ & $-5.15 \%$ & $-2.37 \%$ & 140 & XTW & $0.00 \%$ & $0.00 \%$ & $0.00 \%$ & $0.00 \%$ & $0.00 \%$ \\
\hline
\end{tabular}


Table A9-1. Household energy demand (percentage variations).

\begin{tabular}{|c|c|c|c|c|c|c|c|c|c|c|c|c|c|c|c|c|}
\hline \multirow[b]{2}{*}{ N. } & \multirow[b]{2}{*}{ Code } & \multicolumn{3}{|c|}{$+1^{\circ} \mathrm{C}$} & \multicolumn{3}{|c|}{$+2^{\circ} \mathrm{C}$} & \multicolumn{3}{|c|}{$+3^{\circ} \mathrm{C}$} & & $+4^{\circ} \mathrm{C}$ & & & $+5^{\circ} \mathrm{C}$ & \\
\hline & & Electr. & Gas & Oil.P. & Electr. & Gas & Oil.P. & Electr. & Gas & Oil.P. & Electr. & Gas & Oil.P. & Electr. & Gas & Oil.P. \\
\hline & AUS & $0.00 \%$ & $-0.03 \%$ & $-4.04 \%$ & $0.00 \%$ & $-0.05 \%$ & $-7.98 \%$ & $0.00 \%$ & $-0.08 \%$ & $-11.80 \%$ & $0.00 \%$ & $-0.10 \%$ & $-15.52 \%$ & $0.00 \%$ & $-0.13 \%$ & $-19.15 \%$ \\
\hline & NZL & $0.00 \%$ & $-0.02 \%$ & $-4.74 \%$ & $0.00 \%$ & $-0.04 \%$ & $-9.32 \%$ & $0.00 \%$ & $-0.05 \%$ & $-13.76 \%$ & $0.00 \%$ & $-0.07 \%$ & $-18.06 \%$ & $0.00 \%$ & $-0.09 \%$ & $-22.23 \%$ \\
\hline & $\mathrm{XOC}$ & $0.28 \%$ & $-0.05 \%$ & $-3.35 \%$ & $0.55 \%$ & $-0.11 \%$ & $-6.62 \%$ & $0.82 \%$ & $-0.16 \%$ & $-9.82 \%$ & $1.08 \%$ & $-0.21 \%$ & $-12.94 \%$ & $1.34 \%$ & $-0.26 \%$ & $-16.00 \%$ \\
\hline & $\mathrm{CHN}$ & $-0.06 \%$ & $-0.15 \%$ & $-4.76 \%$ & $-0.11 \%$ & $-0.28 \%$ & $-9.34 \%$ & $-0.15 \%$ & $-0.40 \%$ & $-13.77 \%$ & $-0.19 \%$ & $-0.50 \%$ & $-18.05 \%$ & $-0.23 \%$ & $-0.59 \%$ & $-22.18 \%$ \\
\hline & HKG & $0.28 \%$ & $-0.01 \%$ & $-3.57 \%$ & $0.55 \%$ & $-0.02 \%$ & $-7.06 \%$ & $0.81 \%$ & $-0.03 \%$ & $-10.46 \%$ & $1.07 \%$ & $-0.04 \%$ & $-13.78 \%$ & $1.33 \%$ & $-0.05 \%$ & $-17.03 \%$ \\
\hline & JPN & $-0.04 \%$ & $-0.01 \%$ & $-4.53 \%$ & $-0.07 \%$ & $-0.02 \%$ & $-8.92 \%$ & $-0.10 \%$ & $-0.03 \%$ & $-13.16 \%$ & $-0.12 \%$ & $-0.03 \%$ & $-17.28 \%$ & $-0.15 \%$ & $-0.03 \%$ & $-21.27 \%$ \\
\hline & KOR & $-0.06 \%$ & $-0.12 \%$ & $-4.91 \%$ & $-0.12 \%$ & $-0.22 \%$ & $-9.63 \%$ & $-0.16 \%$ & $-0.30 \%$ & $-14.19 \%$ & $-0.21 \%$ & $-0.37 \%$ & $-18.59 \%$ & $-0.25 \%$ & $-0.43 \%$ & $-22.83 \%$ \\
\hline & MNG & $0.10 \%$ & $-0.75 \%$ & $-8.16 \%$ & $0.21 \%$ & $-1.30 \%$ & $-15.74 \%$ & $0.31 \%$ & $-1.69 \%$ & $-22.80 \%$ & $0.40 \%$ & $-1.96 \%$ & $-29.41 \%$ & $0.50 \%$ & $-2.13 \%$ & $-35.62 \%$ \\
\hline & TWN & $0.29 \%$ & $-0.03 \%$ & $-3.63 \%$ & $0.56 \%$ & $-0.06 \%$ & $-7.18 \%$ & $0.84 \%$ & $-0.09 \%$ & $-10.64 \%$ & $1.10 \%$ & $-0.12 \%$ & $-14.01 \%$ & $1.37 \%$ & $-0.15 \%$ & $-17.31 \%$ \\
\hline 10 & XEA & $-0.12 \%$ & $-0.46 \%$ & $-6.17 \%$ & $-0.23 \%$ & $-0.84 \%$ & $-12.02 \%$ & $-0.32 \%$ & $-1.16 \%$ & $-17.58 \%$ & $-0.41 \%$ & $-1.42 \%$ & $-22.87 \%$ & $-0.48 \%$ & $-1.64 \%$ & $-27.93 \%$ \\
\hline 11 & BRN & $0.27 \%$ & $-0.06 \%$ & $-3.27 \%$ & $0.54 \%$ & $-0.13 \%$ & $-6.46 \%$ & $0.80 \%$ & $-0.19 \%$ & $-9.59 \%$ & $1.06 \%$ & $-0.24 \%$ & $-12.64 \%$ & $1.31 \%$ & $-0.30 \%$ & $-15.64 \%$ \\
\hline 12 & KHM & $0.26 \%$ & $-0.08 \%$ & $-3.24 \%$ & $0.52 \%$ & $-0.16 \%$ & $-6.42 \%$ & $0.78 \%$ & $-0.24 \%$ & $-9.52 \%$ & $1.03 \%$ & $-0.32 \%$ & $-12.56 \%$ & $1.27 \%$ & $-0.39 \%$ & $-15.54 \%$ \\
\hline 13 & IDN & $0.28 \%$ & $-0.08 \%$ & $-3.32 \%$ & $0.55 \%$ & $-0.16 \%$ & $-6.57 \%$ & $0.82 \%$ & $-0.24 \%$ & $-9.74 \%$ & $1.08 \%$ & $-0.32 \%$ & $-12.85 \%$ & $1.33 \%$ & $-0.39 \%$ & $-15.89 \%$ \\
\hline 14 & LAO & $0.27 \%$ & $-0.09 \%$ & $-3.41 \%$ & $0.53 \%$ & $-0.17 \%$ & $-6.73 \%$ & $0.79 \%$ & $-0.25 \%$ & $-9.98 \%$ & $1.04 \%$ & $-0.33 \%$ & $-13.16 \%$ & $1.29 \%$ & $-0.41 \%$ & $-16.27 \%$ \\
\hline & MYS & $0.28 \%$ & $-0.07 \%$ & $-3.30 \%$ & $0.54 \%$ & $-0.13 \%$ & $-6.52 \%$ & $0.81 \%$ & $-0.20 \%$ & $-9.68 \%$ & $1.07 \%$ & $-0.26 \%$ & $-12.76 \%$ & $1.32 \%$ & $-0.32 \%$ & $-15.78 \%$ \\
\hline 16 & PHL & $0.27 \%$ & $-0.07 \%$ & $-3.28 \%$ & $0.54 \%$ & $-0.14 \%$ & $-6.49 \%$ & $0.80 \%$ & $-0.21 \%$ & $-9.62 \%$ & $1.05 \%$ & $-0.27 \%$ & $-12.69 \%$ & $1.30 \%$ & $-0.34 \%$ & $-15.70 \%$ \\
\hline 17 & SGP & $0.27 \%$ & $-0.06 \%$ & $-3.25 \%$ & $0.54 \%$ & $-0.13 \%$ & $-6.44 \%$ & $0.80 \%$ & $-0.19 \%$ & $-9.55 \%$ & $1.05 \%$ & $-0.25 \%$ & $-12.60 \%$ & $1.30 \%$ & $-0.31 \%$ & $-15.58 \%$ \\
\hline 18 & THA & $0.26 \%$ & $-0.09 \%$ & $-3.25 \%$ & $0.52 \%$ & $-0.17 \%$ & $-6.43 \%$ & $0.77 \%$ & $-0.25 \%$ & $-9.53 \%$ & $1.02 \%$ & $-0.33 \%$ & $-12.58 \%$ & $1.26 \%$ & $-0.40 \%$ & $-15.55 \%$ \\
\hline 19 & VNM & $0.27 \%$ & $-0.06 \%$ & $-3.40 \%$ & $0.54 \%$ & $-0.11 \%$ & $-6.72 \%$ & $0.80 \%$ & $-0.17 \%$ & $-9.97 \%$ & $1.05 \%$ & $-0.22 \%$ & $-13.15 \%$ & $1.30 \%$ & $-0.27 \%$ & $-16.25 \%$ \\
\hline 20 & XSE & $0.28 \%$ & $-0.11 \%$ & $-3.42 \%$ & $0.55 \%$ & $-0.22 \%$ & $-6.76 \%$ & $0.82 \%$ & $-0.32 \%$ & $-10.02 \%$ & $1.08 \%$ & $-0.42 \%$ & $-13.22 \%$ & $1.34 \%$ & $-0.52 \%$ & $-16.34 \%$ \\
\hline 21 & $B G D$ & $0.26 \%$ & $-0.13 \%$ & $-3.37 \%$ & $0.51 \%$ & $-0.25 \%$ & $-6.66 \%$ & $0.76 \%$ & $-0.37 \%$ & $-9.88 \%$ & $1.00 \%$ & $-0.48 \%$ & $-13.02 \%$ & $1.24 \%$ & $-0.59 \%$ & $-16.10 \%$ \\
\hline 22 & IND & $0.26 \%$ & $-0.10 \%$ & $-3.42 \%$ & $0.51 \%$ & $-0.20 \%$ & $-6.76 \%$ & $0.76 \%$ & $-0.29 \%$ & $-10.02 \%$ & $1.00 \%$ & $-0.39 \%$ & $-13.21 \%$ & $1.24 \%$ & $-0.47 \%$ & $-16.33 \%$ \\
\hline 23 & NPL & $0.28 \%$ & $-0.10 \%$ & $-3.93 \%$ & $0.56 \%$ & $-0.20 \%$ & $-7.76 \%$ & $0.83 \%$ & $-0.29 \%$ & $-11.48 \%$ & $1.10 \%$ & $-0.38 \%$ & $-15.11 \%$ & $1.36 \%$ & $-0.47 \%$ & $-18.64 \%$ \\
\hline 24 & PAK & $0.26 \%$ & $-0.12 \%$ & $-3.69 \%$ & $0.51 \%$ & $-0.23 \%$ & $-7.29 \%$ & $0.76 \%$ & $-0.33 \%$ & $-10.79 \%$ & $1.01 \%$ & $-0.44 \%$ & $-14.21 \%$ & $1.25 \%$ & $-0.53 \%$ & $-17.55 \%$ \\
\hline 25 & LKA & $0.28 \%$ & $-0.07 \%$ & $-3.35 \%$ & $0.55 \%$ & $-0.14 \%$ & $-6.62 \%$ & $0.81 \%$ & $-0.21 \%$ & $-9.83 \%$ & $1.07 \%$ & $-0.27 \%$ & $-12.96 \%$ & $1.32 \%$ & $-0.34 \%$ & $-16.02 \%$ \\
\hline 26 & XSA & $0.00 \%$ & $-0.04 \%$ & $-3.86 \%$ & $0.00 \%$ & $-0.08 \%$ & $-7.61 \%$ & $-0.01 \%$ & $-0.12 \%$ & $-11.27 \%$ & $-0.01 \%$ & $-0.16 \%$ & $-14.83 \%$ & $-0.01 \%$ & $-0.20 \%$ & $-18.31 \%$ \\
\hline 27 & CAN & $0.08 \%$ & $-1.10 \%$ & $-8.03 \%$ & $0.15 \%$ & $-1.96 \%$ & $-15.47 \%$ & $0.23 \%$ & $-2.64 \%$ & $-22.42 \%$ & $0.31 \%$ & $-3.17 \%$ & $-28.93 \%$ & $0.39 \%$ & $-3.58 \%$ & $-35.04 \%$ \\
\hline 28 & USA & $-0.05 \%$ & $-0.04 \%$ & $-4.87 \%$ & $-0.09 \%$ & $-0.06 \%$ & $-9.57 \%$ & $-0.14 \%$ & $-0.08 \%$ & $-14.10 \%$ & $-0.17 \%$ & $-0.10 \%$ & $-18.48 \%$ & $-0.21 \%$ & $-0.10 \%$ & $-22.72 \%$ \\
\hline & MEX & $0.30 \%$ & $-0.06 \%$ & $-3.73 \%$ & $0.58 \%$ & $-0.13 \%$ & $-7.38 \%$ & $0.86 \%$ & $-0.19 \%$ & $-10.93 \%$ & $1.14 \%$ & $-0.25 \%$ & $-14.39 \%$ & $1.41 \%$ & $-0.31 \%$ & $-17.77 \%$ \\
\hline 30 & XNA & $0.12 \%$ & $0.17 \%$ & $-5.83 \%$ & $0.22 \%$ & $0.33 \%$ & $-11.42 \%$ & $0.33 \%$ & $0.47 \%$ & $-16.80 \%$ & $0.43 \%$ & $0.61 \%$ & $-21.97 \%$ & $0.52 \%$ & $0.74 \%$ & $-26.95 \%$ \\
\hline 31 & ARG & $0.00 \%$ & $-0.01 \%$ & $-4.02 \%$ & $-0.01 \%$ & $-0.03 \%$ & $-7.94 \%$ & $-0.01 \%$ & $-0.05 \%$ & $-11.74 \%$ & $-0.01 \%$ & $-0.06 \%$ & $-15.45 \%$ & $-0.02 \%$ & $-0.08 \%$ & $-19.06 \%$ \\
\hline 32 & $\mathrm{BOL}$ & $0.02 \%$ & $-0.11 \%$ & $-4.11 \%$ & $0.03 \%$ & $-0.21 \%$ & $-8.11 \%$ & $0.05 \%$ & $-0.31 \%$ & $-12.00 \%$ & $0.07 \%$ & $-0.41 \%$ & $-15.78 \%$ & $0.09 \%$ & $-0.51 \%$ & $-19.46 \%$ \\
\hline 33 & BRA & $0.02 \%$ & $-0.08 \%$ & $-3.43 \%$ & $0.04 \%$ & $-0.16 \%$ & $-6.77 \%$ & $0.05 \%$ & $-0.23 \%$ & $-10.04 \%$ & $0.07 \%$ & $-0.31 \%$ & $-13.24 \%$ & $0.09 \%$ & $-0.38 \%$ & $-16.36 \%$ \\
\hline 34 & $\mathrm{CHL}$ & $0.00 \%$ & $0.00 \%$ & $-4.74 \%$ & $0.01 \%$ & $-0.01 \%$ & $-9.33 \%$ & $0.01 \%$ & $-0.02 \%$ & $-13.77 \%$ & $0.02 \%$ & $-0.03 \%$ & $-18.07 \%$ & $0.02 \%$ & $-0.04 \%$ & $-22.25 \%$ \\
\hline 35 & $\mathrm{COL}$ & $0.30 \%$ & $-0.08 \%$ & $-3.50 \%$ & $0.58 \%$ & $-0.15 \%$ & $-6.92 \%$ & $0.87 \%$ & $-0.23 \%$ & $-10.27 \%$ & $1.14 \%$ & $-0.30 \%$ & $-13.53 \%$ & $1.41 \%$ & $-0.37 \%$ & $-16.72 \%$ \\
\hline 36 & ECU & $0.32 \%$ & $-0.08 \%$ & $-3.87 \%$ & $0.64 \%$ & $-0.15 \%$ & $-7.65 \%$ & $0.95 \%$ & $-0.22 \%$ & $-11.32 \%$ & $1.25 \%$ & $-0.29 \%$ & $-14.90 \%$ & $1.54 \%$ & $-0.36 \%$ & $-18.40 \%$ \\
\hline 37 & PRY & $0.01 \%$ & $-0.03 \%$ & $-3.56 \%$ & $0.01 \%$ & $-0.06 \%$ & $-7.03 \%$ & $0.02 \%$ & $-0.08 \%$ & $-10.41 \%$ & $0.02 \%$ & $-0.11 \%$ & $-13.72 \%$ & $0.03 \%$ & $-0.14 \%$ & $-16.95 \%$ \\
\hline 38 & PER & $0.36 \%$ & $-0.07 \%$ & $-4.30 \%$ & $0.70 \%$ & $-0.14 \%$ & $-8.47 \%$ & $1.04 \%$ & $-0.20 \%$ & $-12.52 \%$ & $1.36 \%$ & $-0.26 \%$ & $-16.46 \%$ & $1.68 \%$ & $-0.33 \%$ & $-20.29 \%$ \\
\hline 39 & URY & $0.00 \%$ & $0.01 \%$ & $-4.11 \%$ & $-0.01 \%$ & $0.02 \%$ & $-8.10 \%$ & $-0.01 \%$ & $0.02 \%$ & $-11.98 \%$ & $-0.01 \%$ & $0.03 \%$ & $-15.75 \%$ & $-0.01 \%$ & $0.03 \%$ & $-19.43 \%$ \\
\hline 40 & VEN & $0.29 \%$ & $-0.10 \%$ & $-3.47 \%$ & $0.58 \%$ & $-0.19 \%$ & $-6.85 \%$ & $0.85 \%$ & $-0.28 \%$ & $-10.16 \%$ & $1.13 \%$ & $-0.37 \%$ & $-13.40 \%$ & $1.39 \%$ & $-0.46 \%$ & $-16.56 \%$ \\
\hline & XSM & $0.31 \%$ & $-0.08 \%$ & $-3.77 \%$ & $0.62 \%$ & $-0.16 \%$ & $-7.44 \%$ & $0.91 \%$ & $-0.23 \%$ & $-11.02 \%$ & $1.20 \%$ & $-0.30 \%$ & $-14.51 \%$ & $1.49 \%$ & $-0.37 \%$ & $-17.92 \%$ \\
\hline 42 & CRI & $0.28 \%$ & $-0.08 \%$ & $-3.42 \%$ & $0.56 \%$ & $-0.15 \%$ & $-6.76 \%$ & $0.83 \%$ & $-0.22 \%$ & $-10.02 \%$ & $1.10 \%$ & $-0.29 \%$ & $-13.22 \%$ & $1.36 \%$ & $-0.36 \%$ & $-16.34 \%$ \\
\hline 43 & GTM & $0.30 \%$ & $-0.09 \%$ & $-3.73 \%$ & $0.60 \%$ & $-0.17 \%$ & $-7.38 \%$ & $0.89 \%$ & $-0.26 \%$ & $-10.93 \%$ & $1.17 \%$ & $-0.34 \%$ & $-14.39 \%$ & $1.45 \%$ & $-0.42 \%$ & $-17.77 \%$ \\
\hline 44 & HND & $0.28 \%$ & $-0.07 \%$ & $-3.35 \%$ & $0.54 \%$ & $-0.14 \%$ & $-6.62 \%$ & $0.81 \%$ & $-0.21 \%$ & $-9.82 \%$ & $1.07 \%$ & $-0.28 \%$ & $-12.95 \%$ & $1.32 \%$ & $-0.34 \%$ & $-16.02 \%$ \\
\hline 45 & NIC & $0.27 \%$ & $-0.08 \%$ & $-3.30 \%$ & $0.54 \%$ & $-0.15 \%$ & $-6.54 \%$ & $0.80 \%$ & $-0.22 \%$ & $-9.70 \%$ & $1.06 \%$ & $-0.29 \%$ & $-12.79 \%$ & $1.31 \%$ & $-0.36 \%$ & $-15.81 \%$ \\
\hline 46 & SLV & $0.29 \%$ & $-0.08 \%$ & $-3.52 \%$ & $0.57 \%$ & $-0.16 \%$ & $-6.96 \%$ & $0.85 \%$ & $-0.23 \%$ & $-10.32 \%$ & $1.12 \%$ & $-0.31 \%$ & $-13.60 \%$ & $1.39 \%$ & $-0.38 \%$ & $-16.80 \%$ \\
\hline 47 & PAN & $0.28 \%$ & $-0.07 \%$ & $-3.32 \%$ & $0.55 \%$ & $-0.14 \%$ & $-6.57 \%$ & $0.81 \%$ & $-0.21 \%$ & $-9.75 \%$ & $1.07 \%$ & $-0.28 \%$ & $-12.85 \%$ & $1.33 \%$ & $-0.34 \%$ & $-15.89 \%$ \\
\hline 48 & XCA & $0.27 \%$ & $-0.07 \%$ & $-3.36 \%$ & $0.54 \%$ & $-0.14 \%$ & $-6.64 \%$ & $0.80 \%$ & $-0.21 \%$ & $-9.84 \%$ & $1.06 \%$ & $-0.27 \%$ & $-12.98 \%$ & $1.31 \%$ & $-0.34 \%$ & $-16.05 \%$ \\
\hline 49 & DOM & $0.28 \%$ & $-0.06 \%$ & $-3.38 \%$ & $0.55 \%$ & $-0.11 \%$ & $-6.68 \%$ & $0.82 \%$ & $-0.17 \%$ & $-9.90 \%$ & $1.08 \%$ & $-0.22 \%$ & $-13.06 \%$ & $1.34 \%$ & $-0.27 \%$ & $-16.15 \%$ \\
\hline 50 & JAM & $0.28 \%$ & $-0.05 \%$ & $-3.34 \%$ & $0.55 \%$ & $-0.10 \%$ & $-6.60 \%$ & $0.82 \%$ & $-0.15 \%$ & $-9.79 \%$ & $1.08 \%$ & $-0.20 \%$ & $-12.91 \%$ & $1.33 \%$ & $-0.24 \%$ & $-15.96 \%$ \\
\hline 51 & PRI & $0.28 \%$ & $-0.05 \%$ & $-3.41 \%$ & $0.56 \%$ & $-0.11 \%$ & $-6.74 \%$ & $0.83 \%$ & $-0.16 \%$ & $-10.00 \%$ & $1.10 \%$ & $-0.21 \%$ & $-13.18 \%$ & $1.36 \%$ & $-0.26 \%$ & $-16.29 \%$ \\
\hline 52 & TTO & $0.27 \%$ & $-0.07 \%$ & $-3.29 \%$ & $0.54 \%$ & $-0.14 \%$ & $-6.51 \%$ & $0.80 \%$ & $-0.21 \%$ & $-9.65 \%$ & $1.06 \%$ & $-0.27 \%$ & $-12.73 \%$ & $1.31 \%$ & $-0.34 \%$ & $-15.75 \%$ \\
\hline 53 & $X C B$ & $0.28 \%$ & $-0.06 \%$ & $-3.30 \%$ & $0.54 \%$ & $-0.11 \%$ & $-6.52 \%$ & $0.81 \%$ & $-0.16 \%$ & $-9.68 \%$ & $1.06 \%$ & $-0.21 \%$ & $-12.77 \%$ & $1.32 \%$ & $-0.27 \%$ & $-15.79 \%$ \\
\hline 54 & AUT & $-0.06 \%$ & $-0.05 \%$ & $-5.78 \%$ & $-0.12 \%$ & $-0.09 \%$ & $-11.32 \%$ & $-0.16 \%$ & $-0.12 \%$ & $-16.63 \%$ & $-0.21 \%$ & $-0.14 \%$ & $-21.72 \%$ & $-0.25 \%$ & $-0.15 \%$ & $-26.62 \%$ \\
\hline 55 & BEL & $-0.03 \%$ & $0.02 \%$ & $-5.30 \%$ & $-0.06 \%$ & $0.04 \%$ & $-10.39 \%$ & $-0.08 \%$ & $0.07 \%$ & $-15.31 \%$ & $-0.10 \%$ & $0.09 \%$ & $-20.05 \%$ & $-0.12 \%$ & $0.11 \%$ & $-24.63 \%$ \\
\hline 56 & CYP & $-0.01 \%$ & $0.01 \%$ & $-3.91 \%$ & $-0.02 \%$ & $0.02 \%$ & $-7.71 \%$ & $-0.03 \%$ & $0.03 \%$ & $-11.41 \%$ & $-0.04 \%$ & $0.04 \%$ & $-15.02 \%$ & $-0.05 \%$ & $0.05 \%$ & $-18.53 \%$ \\
\hline 57 & CZE & $0.08 \%$ & $-0.03 \%$ & $-5.84 \%$ & $0.17 \%$ & $-0.04 \%$ & $-11.44 \%$ & $0.24 \%$ & $-0.04 \%$ & $-16.80 \%$ & $0.32 \%$ & $-0.04 \%$ & $-21.96 \%$ & $0.39 \%$ & $-0.03 \%$ & $-26.92 \%$ \\
\hline & DNK & $0.10 \%$ & $0.12 \%$ & $-5.69 \%$ & $0.19 \%$ & $0.24 \%$ & $-11.16 \%$ & $0.28 \%$ & $0.36 \%$ & $-16.41 \%$ & $0.37 \%$ & $0.46 \%$ & $-21.47 \%$ & $0.45 \%$ & $0.56 \%$ & $-26.34 \%$ \\
\hline 59 & EST & $0.10 \%$ & $0.00 \%$ & $-6.35 \%$ & $0.20 \%$ & $0.02 \%$ & $-12.41 \%$ & $0.30 \%$ & $0.06 \%$ & $-18.19 \%$ & $0.39 \%$ & $0.12 \%$ & $-23.71 \%$ & $0.48 \%$ & $0.18 \%$ & $-28.99 \%$ \\
\hline 60 & FIN & $0.12 \%$ & $-0.24 \%$ & $-7.56 \%$ & $0.23 \%$ & $-0.39 \%$ & $-14.66 \%$ & $0.34 \%$ & $-0.47 \%$ & $-21.35 \%$ & $0.45 \%$ & $-0.50 \%$ & $-27.67 \%$ & $0.55 \%$ & $-0.49 \%$ & $-33.66 \%$ \\
\hline 61 & FRA & $-0.03 \%$ & $0.03 \%$ & $-4.87 \%$ & $-0.05 \%$ & $0.06 \%$ & $-9.57 \%$ & $-0.07 \%$ & $0.09 \%$ & $-14.11 \%$ & $-0.08 \%$ & $0.12 \%$ & $-18.51 \%$ & $-0.10 \%$ & $0.14 \%$ & $-22.77 \%$ \\
\hline 62 & DEU & $-0.05 \%$ & $0.01 \%$ & $-5.50 \%$ & $-0.09 \%$ & $0.02 \%$ & $-10.79 \%$ & $-0.12 \%$ & $0.04 \%$ & $-15.87 \%$ & $-0.15 \%$ & $0.06 \%$ & $-20.76 \%$ & $-0.18 \%$ & $0.08 \%$ & $-25.48 \%$ \\
\hline & GRC & $-0.02 \%$ & $0.05 \%$ & $-4.09 \%$ & $-0.04 \%$ & $0.10 \%$ & $-8.07 \%$ & $-0.05 \%$ & $0.15 \%$ & $-11.93 \%$ & $-0.07 \%$ & $0.20 \%$ & $-15.69 \%$ & $-0.08 \%$ & $0.24 \%$ & $-19.34 \%$ \\
\hline & HUN & $-0.05 \%$ & $-0.09 \%$ & $-5.17 \%$ & $-0.10 \%$ & $-0.16 \%$ & $-10.14 \%$ & $-0.15 \%$ & $-0.22 \%$ & $-14.93 \%$ & $-0.18 \%$ & $-0.27 \%$ & $-19.55 \%$ & $-0.22 \%$ & $-0.32 \%$ & $-24.00 \%$ \\
\hline 65 & IRL & $0.08 \%$ & $0.07 \%$ & $-5.22 \%$ & $0.15 \%$ & $0.12 \%$ & $-10.26 \%$ & $0.23 \%$ & $0.17 \%$ & $-15.12 \%$ & $0.29 \%$ & $0.22 \%$ & $-19.82 \%$ & $0.36 \%$ & $0.26 \%$ & $-24.37 \%$ \\
\hline 66 & ITA & $-0.02 \%$ & $0.04 \%$ & $-4.52 \%$ & $-0.05 \%$ & $0.08 \%$ & $-8.90 \%$ & $-0.07 \%$ & $0.12 \%$ & $-13.14 \%$ & $-0.09 \%$ & $0.16 \%$ & $-17.25 \%$ & $-0.10 \%$ & $0.20 \%$ & $-21.24 \%$ \\
\hline 67 & LVA & $0.10 \%$ & $0.03 \%$ & $-6.14 \%$ & $0.20 \%$ & $0.08 \%$ & $-12.00 \%$ & $0.29 \%$ & $0.14 \%$ & $-17.60 \%$ & $0.38 \%$ & $0.21 \%$ & $-22.98 \%$ & $0.47 \%$ & $0.28 \%$ & $-28.13 \%$ \\
\hline 68 & LTU & $0.09 \%$ & $-0.12 \%$ & $-6.13 \%$ & $0.17 \%$ & $-0.21 \%$ & $-11.98 \%$ & $0.26 \%$ & $-0.28 \%$ & $-17.57 \%$ & $0.33 \%$ & $-0.32 \%$ & $-22.93 \%$ & $0.41 \%$ & $-0.35 \%$ & $-28.06 \%$ \\
\hline & LUX & $-0.04 \%$ & $0.03 \%$ & $-5.42 \%$ & $-0.08 \%$ & $0.06 \%$ & $-10.63 \%$ & $-0.11 \%$ & $0.09 \%$ & $-15.64 \%$ & $-0.14 \%$ & $0.12 \%$ & $-20.47 \%$ & $-0.16 \%$ & $0.15 \%$ & $-25.13 \%$ \\
\hline & MLT & $-0.01 \%$ & $0.05 \%$ & $-3.92 \%$ & $-0.02 \%$ & $0.09 \%$ & $-7.73 \%$ & $-0.02 \%$ & $0.14 \%$ & $-11.44 \%$ & $-0.03 \%$ & $0.17 \%$ & $-15.06 \%$ & $-0.04 \%$ & $0.21 \%$ & $-18.58 \%$ \\
\hline
\end{tabular}


Table A9-2. Household energy demand (percentage variations).

\begin{tabular}{|c|c|c|c|c|c|c|c|c|c|c|c|c|c|c|c|}
\hline \multirow[b]{2}{*}{ Code } & \multicolumn{3}{|c|}{$+1^{\circ} \mathrm{C}$} & \multicolumn{3}{|c|}{$+2^{\circ} \mathrm{C}$} & \multicolumn{3}{|c|}{$+3^{\circ} \mathrm{C}$} & & $+4^{\circ} \mathrm{C}$ & & & $+5^{\circ} \mathrm{C}$ & \\
\hline & Electr. & Gas & Oil.P. & Electr. & Gas & Oil.P. & Electr. & Gas & Oil.P. & Electr. & Gas & Oil.P. & Electr. & Gas & Oil.P. \\
\hline \begin{tabular}{l|l|}
71 & NLD \\
\end{tabular} & $-0.03 \%$ & $0.02 \%$ & $-5.26 \%$ & $-0.05 \%$ & $0.05 \%$ & $-10.32 \%$ & $-0.08 \%$ & $0.07 \%$ & $-15.20 \%$ & $-0.10 \%$ & $0.09 \%$ & $-19.91 \%$ & $-0.11 \%$ & $0.11 \%$ & $-24.46 \%$ \\
\hline $72 \mid \mathrm{POL}$ & $0.08 \%$ & $-0.07 \%$ & $-5.87 \%$ & $0.17 \%$ & $-0.12 \%$ & $-11.48 \%$ & $0.24 \%$ & $-0.16 \%$ & $-16.87 \%$ & $0.32 \%$ & $-0.18 \%$ & $-22.04 \%$ & $0.39 \%$ & $-0.19 \%$ & $-27.01 \%$ \\
\hline $73 \mid$ PRT & $0.00 \%$ & $0.02 \%$ & $-4.24 \%$ & $0.00 \%$ & $0.03 \%$ & $-8.36 \%$ & $0.00 \%$ & $0.04 \%$ & $-12.36 \%$ & $0.00 \%$ & $0.05 \%$ & $-16.25 \%$ & $0.00 \%$ & $0.06 \%$ & $-20.03 \%$ \\
\hline 74 SVK & $-0.07 \%$ & $-0.12 \%$ & $-6.02 \%$ & $-0.13 \%$ & $-0.21 \%$ & $-11.77 \%$ & $-0.18 \%$ & $-0.29 \%$ & $-17.28 \%$ & $-0.23 \%$ & $-0.35 \%$ & $-22.55 \%$ & $-0.28 \%$ & $-0.39 \%$ & $-27.60 \%$ \\
\hline 75 SVN & $-0.05 \%$ & $-0.04 \%$ & $-5.54 \%$ & $-0.10 \%$ & $-0.07 \%$ & $-10.85 \%$ & $-0.14 \%$ & $-0.09 \%$ & $-15.96 \%$ & $-0.18 \%$ & $-0.11 \%$ & $-20.88 \%$ & $-0.21 \%$ & $-0.12 \%$ & $-25.61 \%$ \\
\hline $76 \mid$ ESP & $-0.01 \%$ & $0.04 \%$ & $-4.25 \%$ & $-0.02 \%$ & $0.08 \%$ & $-8.38 \%$ & $-0.03 \%$ & $0.11 \%$ & $-12.39 \%$ & $-0.03 \%$ & $0.15 \%$ & $-16.29 \%$ & $-0.04 \%$ & $0.17 \%$ & $-20.07 \%$ \\
\hline 77 SWE & $0.11 \%$ & $0.07 \%$ & $-6.59 \%$ & $0.22 \%$ & $0.15 \%$ & $-12.87 \%$ & $0.32 \%$ & $0.24 \%$ & $-18.85 \%$ & $0.42 \%$ & $0.35 \%$ & $-24.55 \%$ & $0.52 \%$ & $0.46 \%$ & $-30.01 \%$ \\
\hline 78 GBR & $0.08 \%$ & $0.07 \%$ & $-5.30 \%$ & $0.16 \%$ & $0.14 \%$ & $-10.42 \%$ & $0.23 \%$ & $0.19 \%$ & $-15.35 \%$ & $0.31 \%$ & $0.25 \%$ & $-20.12 \%$ & $0.38 \%$ & $0.29 \%$ & $-24.72 \%$ \\
\hline $79 \mathrm{CHE}$ & $-0.06 \%$ & $-0.02 \%$ & $-6.02 \%$ & $-0.11 \%$ & $-0.04 \%$ & $-11.77 \%$ & $-0.16 \%$ & $-0.04 \%$ & $-17.28 \%$ & $-0.20 \%$ & $-0.04 \%$ & $-22.57 \%$ & $-0.23 \%$ & $-0.03 \%$ & $-27.64 \%$ \\
\hline 80 NOR & $0.11 \%$ & $0.05 \%$ & $-6.55 \%$ & $0.21 \%$ & $0.12 \%$ & $-12.80 \%$ & $0.31 \%$ & $0.19 \%$ & $-18.76 \%$ & $0.40 \%$ & $0.26 \%$ & $-24.46 \%$ & $0.49 \%$ & $0.34 \%$ & $-29.92 \%$ \\
\hline 81 XEF & $0.09 \%$ & $0.07 \%$ & $-5.89 \%$ & $0.18 \%$ & $0.14 \%$ & $-11.54 \%$ & $0.27 \%$ & $0.21 \%$ & $-16.97 \%$ & $0.35 \%$ & $0.27 \%$ & $-22.19 \%$ & $0.43 \%$ & $0.33 \%$ & $-27.21 \%$ \\
\hline 82 ALB & $-0.03 \%$ & $0.06 \%$ & $-4.44 \%$ & $-0.05 \%$ & $0.12 \%$ & $-8.75 \%$ & $-0.07 \%$ & $0.17 \%$ & $-12.92 \%$ & $-0.09 \%$ & $0.22 \%$ & $-16.97 \%$ & $-0.11 \%$ & $0.27 \%$ & $-20.90 \%$ \\
\hline $83 \mid \mathrm{BGR}$ & $-0.05 \%$ & $-0.05 \%$ & $-5.25 \%$ & $-0.10 \%$ & $-0.10 \%$ & $-10.31 \%$ & $-0.14 \%$ & $-0.13 \%$ & $-15.17 \%$ & $-0.18 \%$ & $-0.16 \%$ & $-19.86 \%$ & $-0.21 \%$ & $-0.18 \%$ & $-24.38 \%$ \\
\hline 84 BLR & $0.08 \%$ & $-0.28 \%$ & $-6.36 \%$ & $0.16 \%$ & $-0.49 \%$ & $-12.41 \%$ & $0.24 \%$ & $-0.66 \%$ & $-18.16 \%$ & $0.31 \%$ & $-0.78 \%$ & $-23.65 \%$ & $0.38 \%$ & $-0.87 \%$ & $-28.89 \%$ \\
\hline 85 HRV & $-0.03 \%$ & $0.01 \%$ & $-4.66 \%$ & $-0.06 \%$ & $0.02 \%$ & $-9.17 \%$ & $-0.09 \%$ & $0.03 \%$ & $-13.54 \%$ & $-0.11 \%$ & $0.05 \%$ & $-17.77 \%$ & $-0.14 \%$ & $0.06 \%$ & $-21.86 \%$ \\
\hline 86 ROU & $-0.06 \%$ & $-0.12 \%$ & $-5.51 \%$ & $-0.12 \%$ & $-0.23 \%$ & $-10.80 \%$ & $-0.17 \%$ & $-0.31 \%$ & $-15.87 \%$ & $-0.21 \%$ & $-0.38 \%$ & $-20.75 \%$ & $-0.25 \%$ & $-0.44 \%$ & $-25.45 \%$ \\
\hline 87 RUS & $0.10 \%$ & $-0.82 \%$ & $-8.24 \%$ & $0.20 \%$ & $-1.44 \%$ & $-15.89 \%$ & $0.29 \%$ & $-1.90 \%$ & $-23.02 \%$ & $0.39 \%$ & $-2.23 \%$ & $-29.70 \%$ & $0.48 \%$ & $-2.45 \%$ & $-35.97 \%$ \\
\hline 88 UKR & $-0.08 \%$ & $-0.11 \%$ & $-5.51 \%$ & $-0.14 \%$ & $-0.19 \%$ & $-10.79 \%$ & $-0.21 \%$ & $-0.26 \%$ & $-15.86 \%$ & $-0.26 \%$ & $-0.31 \%$ & $-20.72 \%$ & $-0.31 \%$ & $-0.34 \%$ & $-25.40 \%$ \\
\hline 89 XEE & $-0.07 \%$ & $-0.16 \%$ & $-5.33 \%$ & $-0.13 \%$ & $-0.29 \%$ & $-10.45 \%$ & $-0.18 \%$ & $-0.41 \%$ & $-15.37 \%$ & $-0.23 \%$ & $-0.50 \%$ & $-20.10 \%$ & $-0.28 \%$ & $-0.58 \%$ & $-24.66 \%$ \\
\hline 90 XER & $-0.03 \%$ & $0.01 \%$ & $-4.95 \%$ & $-0.05 \%$ & $0.02 \%$ & $-9.74 \%$ & $-0.07 \%$ & $0.03 \%$ & $-14.36 \%$ & $-0.09 \%$ & $0.04 \%$ & $-18.83 \%$ & $-0.11 \%$ & $0.05 \%$ & $-23.15 \%$ \\
\hline $91 \mid$ KAZ & $-0.20 \%$ & $-0.98 \%$ & $-7.30 \%$ & $-0.37 \%$ & $-1.76 \%$ & $-14.08 \%$ & $-0.52 \%$ & $-2.39 \%$ & $-20.39 \%$ & $-0.65 \%$ & $-2.88 \%$ & $-26.31 \%$ & $-0.77 \%$ & $-3.27 \%$ & $-31.88 \%$ \\
\hline 92 KGZ & $-0.11 \%$ & $-0.45 \%$ & $-5.93 \%$ & $-0.20 \%$ & $-0.83 \%$ & $-11.56 \%$ & $-0.29 \%$ & $-1.16 \%$ & $-16.93 \%$ & $-0.36 \%$ & $-1.43 \%$ & $-22.05 \%$ & $-0.43 \%$ & $-1.66 \%$ & $-26.95 \%$ \\
\hline 93 XSU & $-0.06 \%$ & $-0.11 \%$ & $-4.63 \%$ & $-0.11 \%$ & $-0.20 \%$ & $-9.10 \%$ & $-0.16 \%$ & $-0.27 \%$ & $-13.42 \%$ & $-0.20 \%$ & $-0.34 \%$ & $-17.59 \%$ & $-0.24 \%$ & $-0.39 \%$ & $-21.63 \%$ \\
\hline 94 ARM & $-0.10 \%$ & $-0.30 \%$ & $-6.07 \%$ & $-0.19 \%$ & $-0.55 \%$ & $-11.86 \%$ & $-0.27 \%$ & $-0.76 \%$ & $-17.37 \%$ & $-0.34 \%$ & $-0.93 \%$ & $-22.64 \%$ & $-0.41 \%$ & $-1.08 \%$ & $-27.67 \%$ \\
\hline 95 AZE & $-0.05 \%$ & $-0.05 \%$ & $-4.71 \%$ & $-0.10 \%$ & $-0.09 \%$ & $-9.26 \%$ & $-0.14 \%$ & $-0.12 \%$ & $-13.65 \%$ & $-0.18 \%$ & $-0.15 \%$ & $-17.90 \%$ & $-0.21 \%$ & $-0.17 \%$ & $-22.01 \%$ \\
\hline 96 GEO & $-0.03 \%$ & $0.02 \%$ & $-4.53 \%$ & $-0.06 \%$ & $0.04 \%$ & $-8.92 \%$ & $-0.08 \%$ & $0.06 \%$ & $-13.17 \%$ & $-0.10 \%$ & $0.08 \%$ & $-17.29 \%$ & $-0.12 \%$ & $0.10 \%$ & $-21.28 \%$ \\
\hline $97 \mathrm{BHR}$ & $0.01 \%$ & $-0.17 \%$ & $-3.47 \%$ & $0.01 \%$ & $-0.34 \%$ & $-6.85 \%$ & $0.02 \%$ & $-0.49 \%$ & $-10.16 \%$ & $0.02 \%$ & $-0.65 \%$ & $-13.39 \%$ & $0.03 \%$ & $-0.79 \%$ & $-16.55 \%$ \\
\hline 98 IRN & $-0.04 \%$ & $-0.05 \%$ & $-4.25 \%$ & $-0.08 \%$ & $-0.09 \%$ & $-8.36 \%$ & $-0.11 \%$ & $-0.13 \%$ & $-12.35 \%$ & $-0.14 \%$ & $-0.16 \%$ & $-16.23 \%$ & $-0.17 \%$ & $-0.19 \%$ & $-19.99 \%$ \\
\hline 99 ISR & $-0.01 \%$ & $-0.04 \%$ & $-3.89 \%$ & $-0.02 \%$ & $-0.09 \%$ & $-7.67 \%$ & $-0.03 \%$ & $-0.13 \%$ & $-11.36 \%$ & $-0.03 \%$ & $-0.17 \%$ & $-14.94 \%$ & $-0.04 \%$ & $-0.20 \%$ & $-18.44 \%$ \\
\hline 100/JOR & $-0.02 \%$ & $-0.05 \%$ & $-3.95 \%$ & $-0.03 \%$ & $-0.10 \%$ & $-7.79 \%$ & $-0.05 \%$ & $-0.15 \%$ & $-11.52 \%$ & $-0.06 \%$ & $-0.19 \%$ & $-15.16 \%$ & $-0.08 \%$ & $-0.24 \%$ & $-18.70 \%$ \\
\hline 101 KWT & $0.00 \%$ & $-0.21 \%$ & $-3.59 \%$ & $0.00 \%$ & $-0.41 \%$ & $-7.10 \%$ & $-0.01 \%$ & $-0.60 \%$ & $-10.52 \%$ & $-0.01 \%$ & $-0.78 \%$ & $-13.85 \%$ & $-0.01 \%$ & $-0.96 \%$ & $-17.11 \%$ \\
\hline 102 OMN & $0.26 \%$ & $-0.13 \%$ & $-3.36 \%$ & $0.51 \%$ & $-0.26 \%$ & $-6.64 \%$ & $0.76 \%$ & $-0.38 \%$ & $-9.85 \%$ & $1.00 \%$ & $-0.50 \%$ & $-12.99 \%$ & $1.24 \%$ & $-0.61 \%$ & $-16.06 \%$ \\
\hline 103 QAT & $0.01 \%$ & $-0.19 \%$ & $-3.43 \%$ & $0.02 \%$ & $-0.36 \%$ & $-6.77 \%$ & $0.02 \%$ & $-0.54 \%$ & $-10.04 \%$ & $0.03 \%$ & $-0.70 \%$ & $-13.24 \%$ & $0.04 \%$ & $-0.86 \%$ & $-16.37 \%$ \\
\hline 104 SAU & $0.00 \%$ & $-0.16 \%$ & $-3.49 \%$ & $0.01 \%$ & $-0.31 \%$ & $-6.89 \%$ & $0.01 \%$ & $-0.46 \%$ & $-10.22 \%$ & $0.02 \%$ & $-0.60 \%$ & $-13.47 \%$ & $0.02 \%$ & $-0.74 \%$ & $-16.64 \%$ \\
\hline 106 TUR & $-0.04 \%$ & $-0.01 \%$ & $-4.70 \%$ & $-0.08 \%$ & $-0.01 \%$ & $-9.24 \%$ & $-0.11 \%$ & $-0.02 \%$ & $-13.64 \%$ & $-0.14 \%$ & $-0.02 \%$ & $-17.89 \%$ & $-0.16 \%$ & $-0.01 \%$ & $-22.01 \%$ \\
\hline 105 ARE & $0.26 \%$ & $-0.14 \%$ & $-3.40 \%$ & $0.52 \%$ & $-0.27 \%$ & $-6.72 \%$ & $0.77 \%$ & $-0.40 \%$ & $-9.97 \%$ & $1.02 \%$ & $-0.53 \%$ & $-13.15 \%$ & $1.26 \%$ & $-0.65 \%$ & $-16.25 \%$ \\
\hline 107 XWS & $0.29 \%$ & $-0.01 \%$ & $-3.73 \%$ & $0.56 \%$ & $-0.03 \%$ & $-7.37 \%$ & $0.84 \%$ & $-0.04 \%$ & $-10.92 \%$ & $1.10 \%$ & $-0.05 \%$ & $-14.38 \%$ & $1.36 \%$ & $-0.06 \%$ & $-17.75 \%$ \\
\hline 108 EGY & $-0.01 \%$ & $-0.05 \%$ & $-3.64 \%$ & $-0.01 \%$ & $-0.10 \%$ & $-7.19 \%$ & $-0.01 \%$ & $-0.15 \%$ & $-10.65 \%$ & $-0.02 \%$ & $-0.19 \%$ & $-14.03 \%$ & $-0.02 \%$ & $-0.24 \%$ & $-17.32 \%$ \\
\hline 109 MAR & $-0.01 \%$ & $0.03 \%$ & $-4.04 \%$ & $-0.02 \%$ & $0.05 \%$ & $-7.98 \%$ & $-0.02 \%$ & $0.07 \%$ & $-11.80 \%$ & $-0.03 \%$ & $0.09 \%$ & $-15.52 \%$ & $-0.03 \%$ & $0.11 \%$ & $-19.15 \%$ \\
\hline 110TUN & $-0.01 \%$ & $0.03 \%$ & $-3.95 \%$ & $-0.03 \%$ & $0.07 \%$ & $-7.79 \%$ & $-0.04 \%$ & $0.09 \%$ & $-11.53 \%$ & $-0.05 \%$ & $0.12 \%$ & $-15.17 \%$ & $-0.06 \%$ & $0.15 \%$ & $-18.72 \%$ \\
\hline 111 XNF & $-0.01 \%$ & $0.01 \%$ & $-3.90 \%$ & $-0.03 \%$ & $0.02 \%$ & $-7.69 \%$ & $-0.04 \%$ & $0.02 \%$ & $-11.38 \%$ & $-0.05 \%$ & $0.03 \%$ & $-14.97 \%$ & $-0.06 \%$ & $0.04 \%$ & $-18.48 \%$ \\
\hline 112 BEN & $0.27 \%$ & $-0.11 \%$ & $-3.28 \%$ & $0.54 \%$ & $-0.22 \%$ & $-6.48 \%$ & $0.80 \%$ & $-0.32 \%$ & $-9.62 \%$ & $1.05 \%$ & $-0.42 \%$ & $-12.69 \%$ & $1.30 \%$ & $-0.52 \%$ & $-15.69 \%$ \\
\hline 113 BFA & $0.25 \%$ & $-0.14 \%$ & $-3.18 \%$ & $0.50 \%$ & $-0.27 \%$ & $-6.30 \%$ & $0.74 \%$ & $-0.40 \%$ & $-9.35 \%$ & $0.98 \%$ & $-0.53 \%$ & $-12.33 \%$ & $1.21 \%$ & $-0.65 \%$ & $-15.25 \%$ \\
\hline $114 \mathrm{CMR}$ & $0.29 \%$ & $-0.10 \%$ & $-3.51 \%$ & $0.58 \%$ & $-0.21 \%$ & $-6.94 \%$ & $0.85 \%$ & $-0.30 \%$ & $-10.29 \%$ & $1.13 \%$ & $-0.40 \%$ & $-13.56 \%$ & $1.39 \%$ & $-0.49 \%$ & $-16.75 \%$ \\
\hline $115 \mathrm{CIV}$ & $0.28 \%$ & $-0.10 \%$ & $-3.34 \%$ & $0.55 \%$ & $-0.20 \%$ & $-6.60 \%$ & $0.81 \%$ & $-0.30 \%$ & $-9.80 \%$ & $1.07 \%$ & $-0.40 \%$ & $-12.92 \%$ & $1.33 \%$ & $-0.49 \%$ & $-15.97 \%$ \\
\hline $116 \mathrm{GHA}$ & $0.28 \%$ & $-0.10 \%$ & $-3.30 \%$ & $0.55 \%$ & $-0.20 \%$ & $-6.53 \%$ & $0.81 \%$ & $-0.29 \%$ & $-9.69 \%$ & $1.07 \%$ & $-0.39 \%$ & $-12.78 \%$ & $1.32 \%$ & $-0.47 \%$ & $-15.81 \%$ \\
\hline 117 GIN & $0.27 \%$ & $-0.11 \%$ & $-3.37 \%$ & $0.54 \%$ & $-0.21 \%$ & $-6.66 \%$ & $0.81 \%$ & $-0.32 \%$ & $-9.89 \%$ & $1.06 \%$ & $-0.42 \%$ & $-13.04 \%$ & $1.32 \%$ & $-0.51 \%$ & $-16.12 \%$ \\
\hline 118 NGA & $0.27 \%$ & $-0.12 \%$ & $-3.29 \%$ & $0.53 \%$ & $-0.23 \%$ & $-6.50 \%$ & $0.78 \%$ & $-0.34 \%$ & $-9.65 \%$ & $1.03 \%$ & $-0.44 \%$ & $-12.73 \%$ & $1.28 \%$ & $-0.55 \%$ & $-15.74 \%$ \\
\hline 119 SEN & $0.26 \%$ & $-0.11 \%$ & $-3.26 \%$ & $0.52 \%$ & $-0.21 \%$ & $-6.44 \%$ & $0.77 \%$ & $-0.32 \%$ & $-9.56 \%$ & $1.02 \%$ & $-0.41 \%$ & $-12.60 \%$ & $1.26 \%$ & $-0.51 \%$ & $-15.59 \%$ \\
\hline 120 TGO & $0.28 \%$ & $-0.11 \%$ & $-3.32 \%$ & $0.54 \%$ & $-0.22 \%$ & $-6.56 \%$ & $0.81 \%$ & $-0.32 \%$ & $-9.73 \%$ & $1.07 \%$ & $-0.42 \%$ & $-12.83 \%$ & $1.32 \%$ & $-0.52 \%$ & $-15.87 \%$ \\
\hline 121 XWF & $0.27 \%$ & $-0.10 \%$ & $-3.36 \%$ & $0.54 \%$ & $-0.19 \%$ & $-6.65 \%$ & $0.80 \%$ & $-0.28 \%$ & $-9.87 \%$ & $1.06 \%$ & $-0.37 \%$ & $-13.01 \%$ & $1.31 \%$ & $-0.46 \%$ & $-16.09 \%$ \\
\hline 122 XCF & $0.28 \%$ & $-0.11 \%$ & $-3.41 \%$ & $0.56 \%$ & $-0.21 \%$ & $-6.74 \%$ & $0.83 \%$ & $-0.32 \%$ & $-10.00 \%$ & $1.09 \%$ & $-0.42 \%$ & $-13.18 \%$ & $1.35 \%$ & $-0.51 \%$ & $-16.29 \%$ \\
\hline 123 XAC & $0.30 \%$ & $-0.09 \%$ & $-3.62 \%$ & $0.59 \%$ & $-0.18 \%$ & $-7.15 \%$ & $0.87 \%$ & $-0.27 \%$ & $-10.60 \%$ & $1.15 \%$ & $-0.35 \%$ & $-13.97 \%$ & $1.42 \%$ & $-0.43 \%$ & $-17.25 \%$ \\
\hline 124 ETH & $0.34 \%$ & $-0.12 \%$ & $-3.98 \%$ & $0.67 \%$ & $-0.23 \%$ & $-7.86 \%$ & $0.99 \%$ & $-0.34 \%$ & $-11.63 \%$ & $1.30 \%$ & $-0.44 \%$ & $-15.31 \%$ & $1.61 \%$ & $-0.54 \%$ & $-18.89 \%$ \\
\hline 125 KEN & $0.31 \%$ & $-0.09 \%$ & $-3.77 \%$ & $0.62 \%$ & $-0.17 \%$ & $-7.45 \%$ & $0.92 \%$ & $-0.25 \%$ & $-11.03 \%$ & $1.21 \%$ & $-0.33 \%$ & $-14.53 \%$ & $1.49 \%$ & $-0.41 \%$ & $-17.94 \%$ \\
\hline 126 MDG & $0.30 \%$ & $-0.06 \%$ & $-3.64 \%$ & $0.59 \%$ & $-0.11 \%$ & $-7.20 \%$ & $0.87 \%$ & $-0.17 \%$ & $-10.66 \%$ & $1.15 \%$ & $-0.22 \%$ & $-14.05 \%$ & $1.42 \%$ & $-0.28 \%$ & $-17.35 \%$ \\
\hline $127 \mathrm{MWI}$ & $0.29 \%$ & $-0.10 \%$ & $-3.68 \%$ & $0.57 \%$ & $-0.20 \%$ & $-7.27 \%$ & $0.85 \%$ & $-0.30 \%$ & $-10.77 \%$ & $1.12 \%$ & $-0.40 \%$ & $-14.19 \%$ & $1.38 \%$ & $-0.49 \%$ & $-17.52 \%$ \\
\hline 128 MUS & $0.29 \%$ & $-0.04 \%$ & $-3.49 \%$ & $0.57 \%$ & $-0.08 \%$ & $-6.89 \%$ & $0.85 \%$ & $-0.12 \%$ & $-10.21 \%$ & $1.12 \%$ & $-0.16 \%$ & $-13.46 \%$ & $1.38 \%$ & $-0.20 \%$ & $-16.64 \%$ \\
\hline $129 \mathrm{MOZ}$ & $0.28 \%$ & $-0.07 \%$ & $-3.48 \%$ & $0.55 \%$ & $-0.13 \%$ & $-6.89 \%$ & $0.82 \%$ & $-0.20 \%$ & $-10.21 \%$ & $1.08 \%$ & $-0.26 \%$ & $-13.46 \%$ & $1.34 \%$ & $-0.32 \%$ & $-16.63 \%$ \\
\hline 130 RWA & $0.33 \%$ & $-0.08 \%$ & $-3.95 \%$ & $0.65 \%$ & $-0.15 \%$ & $-7.79 \%$ & $0.97 \%$ & $-0.22 \%$ & $-11.53 \%$ & $1.27 \%$ & $-0.29 \%$ & $-15.18 \%$ & $1.57 \%$ & $-0.36 \%$ & $-18.73 \%$ \\
\hline 131 TZA & $0.30 \%$ & $-0.08 \%$ & $-3.62 \%$ & $0.59 \%$ & $-0.16 \%$ & $-7.14 \%$ & $0.87 \%$ & $-0.24 \%$ & $-10.59 \%$ & $1.14 \%$ & $-0.31 \%$ & $-13.95 \%$ & $1.41 \%$ & $-0.38 \%$ & $-17.24 \%$ \\
\hline 132 UGA & $0.31 \%$ & $-0.07 \%$ & $-3.70 \%$ & $0.61 \%$ & $-0.14 \%$ & $-7.32 \%$ & $0.91 \%$ & $-0.21 \%$ & $-10.84 \%$ & $1.20 \%$ & $-0.27 \%$ & $-14.28 \%$ & $1.48 \%$ & $-0.33 \%$ & $-17.64 \%$ \\
\hline $133 \mathrm{ZMB}$ & $0.29 \%$ & $-0.12 \%$ & $-3.72 \%$ & $0.57 \%$ & $-0.24 \%$ & $-7.34 \%$ & $0.85 \%$ & $-0.36 \%$ & $-10.88 \%$ & $1.12 \%$ & $-0.47 \%$ & $-14.33 \%$ & $1.38 \%$ & $-0.58 \%$ & $-17.70 \%$ \\
\hline 134 ZWE & $0.29 \%$ & $-0.10 \%$ & $-3.82 \%$ & $0.58 \%$ & $-0.20 \%$ & $-7.54 \%$ & $0.86 \%$ & $-0.30 \%$ & $-11.17 \%$ & $1.14 \%$ & $-0.39 \%$ & $-14.71 \%$ & $1.40 \%$ & $-0.48 \%$ & $-18.16 \%$ \\
\hline 135 XEC & $0.29 \%$ & $-0.09 \%$ & $-3.43 \%$ & $0.57 \%$ & $-0.19 \%$ & $-6.77 \%$ & $0.85 \%$ & $-0.27 \%$ & $-10.05 \%$ & $1.12 \%$ & $-0.36 \%$ & $-13.24 \%$ & $1.38 \%$ & $-0.44 \%$ & $-16.37 \%$ \\
\hline 136 BWA & $0.27 \%$ & $-0.08 \%$ & $-3.68 \%$ & $0.54 \%$ & $-0.16 \%$ & $-7.26 \%$ & $0.81 \%$ & $-0.23 \%$ & $-10.75 \%$ & $1.06 \%$ & $-0.30 \%$ & $-14.17 \%$ & $1.32 \%$ & $-0.37 \%$ & $-17.49 \%$ \\
\hline 137NAM & $0.29 \%$ & $-0.07 \%$ & $-3.77 \%$ & $0.57 \%$ & $-0.14 \%$ & $-7.44 \%$ & $0.85 \%$ & $-0.20 \%$ & $-11.02 \%$ & $1.12 \%$ & $-0.27 \%$ & $-14.51 \%$ & $1.39 \%$ & $-0.33 \%$ & $-17.91 \%$ \\
\hline $138 \mathrm{ZAF}$ & $0.00 \%$ & $-0.06 \%$ & $-4.08 \%$ & $0.01 \%$ & $-0.11 \%$ & $-8.05 \%$ & $0.01 \%$ & $-0.16 \%$ & $-11.91 \%$ & $0.01 \%$ & $-0.21 \%$ & $-15.67 \%$ & $0.02 \%$ & $-0.26 \%$ & $-19.32 \%$ \\
\hline 139 XSC & $0.00 \%$ & $-0.05 \%$ & $-4.13 \%$ & $0.01 \%$ & $-0.11 \%$ & $-8.14 \%$ & $0.01 \%$ & $-0.16 \%$ & $-12.04 \%$ & $0.02 \%$ & $-0.21 \%$ & $-15.84 \%$ & $0.02 \%$ & $-0.25 \%$ & $-19.54 \%$ \\
\hline 140XTW & $0.12 \%$ & $-0.26 \%$ & $-7.58 \%$ & $0.23 \%$ & $-0.45 \%$ & $-14.72 \%$ & $0.33 \%$ & $-0.56 \%$ & $-21.44 \%$ & $0.44 \%$ & $-0.63 \%$ & $-27.81 \%$ & $0.53 \%$ & $-0.66 \%$ & $-33.83 \%$ \\
\hline
\end{tabular}

